\title{
Designing Size Consistent Statistics for Accurate Anomaly Detection in Dynamic Networks
}

\author{
TIMOTHY LA FOND, Lawrence Livermore National Laboratory \\ JENNIFER NEVILLE, Purdue University \\ BRIAN GALLAGHER, Lawrence Livermore National Laboratory
}

\begin{abstract}
An important task in network analysis is the detection of anomalous events in a network time series. These events could merely be times of interest in the network timeline or they could be examples of malicious activity or network malfunction. Hypothesis testing using network statistics to summarize the behavior of the network provides a robust framework for the anomaly detection decision process. Unfortunately, choosing network statistics that are dependent on confounding factors like the total number of nodes or edges can lead to incorrect conclusions (e.g., false positives and false negatives). In this article, we describe the challenges that face anomaly detection in dynamic network streams regarding confounding factors. We also provide two solutions to avoiding error due to confounding factors: the first is a randomization testing method that controls for confounding factors, and the second is a set of size-consistent network statistics that avoid confounding due to the most common factors, edge count and node count.
\end{abstract}

CCS Concepts: $\bullet$ Mathematics of computing $\rightarrow$ Time series analysis; $\bullet$ Computing methodologies $\rightarrow$ Anomaly detection;

Additional Key Words and Phrases: Graphs, statistics, data mining

ACM Reference format:

Timothy La Fond, Jennifer Neville, and Brian Gallagher. 2018. Designing Size Consistent Statistics for Accurate Anomaly Detection in Dynamic Networks. ACM Trans. Knowl. Discov. Data. 12, 4, Article 46 (April 2018), 49 pages.

https://doi.org/10.1145/3185059

\section{INTRODUCTION}

Dynamic networks are a common form of data, which include such sources as social networks, computer communications, and biological interactions. Like typical networks, dynamic networks consist of a set of entities (nodes) and links between them (edges), which indicate interactions or connections. However, the key aspect of dynamic networks is that their topological structure changes over time. For example, in a social network, individuals may add new friends or send new messages or new users could appear in the network as time passes.

Many network changes are gradual, as the networks evolve slowly over time. Sometimes, though, networks experience sudden changes in behavior, which are often temporary as well.

This research is supported by NSF under Contract numbers IIS-1149789, CCF-0939370, IIS-1546488, and IIS-1618690. This work was performed under the auspices of the US Department of Energy by Lawrence Livermore National Laboratory under Contract DE-AC52-07NA27344.

Authors' addresses: T. La Fond and B. Gallagher, 7000 East Ave, Livermore, CA 94550; emails: \{lafond1, gallagher23\}@llnl.gov; J. Neville, 610 Purdue Mall, West Lafayette, IN 47907; email: neville@cs.purdue.edu.

Permission to make digital or hard copies of all or part of this work for personal or classroom use is granted without fee provided that copies are not made or distributed for profit or commercial advantage and that copies bear this notice and the full citation on the first page. Copyrights for components of this work owned by others than ACM must be honored. Abstracting with credit is permitted. To copy otherwise, or republish, to post on servers or to redistribute to lists, requires prior specific permission and/or a fee. Request permissions from permissions@acm.org.

(c) 2018 ACM 1556-4681/2018/04-ART46 \$15.00

https://doi.org/10.1145/3185059 
These changes are referred to as Anomalies and can be caused by an event the network is experiencing (some much-discussed news in a social network, for example) or may be indicative of some kind of problem or fault (such as a loss of routers in a computer network). Detecting when anomalies occur in dynamic networks, a task known as Dynamic Network Anomaly Detection, is critical in a number of different domains and is the focus of this research. The formal definition of this task, as well as the process by which dynamic network data is observed, is described in Section 2.

A statistical approach to dynamic network anomaly detection requires a Network Statistic, which summarizes the properties of the graph at a certain time. Network statistics are used to convert a sequence of graph structures into a sequence of statistic values, which are easier to analyze for change points. An example of a network statistic is the Clustering Coefficient, which measures the transitivity (preference for triangles) of a graph.

However, not all changes in network structure indicate anomalous events. A typical real-world network experiences many changes in the course of its normal behavior: the network can grow in size as time passes, or the quantity of messages being sent in a social network could vary randomly from day-to-day. These non-anomalous changes add noise for the anomaly detector. In addition, network statistics designed to measure properties other than total message/node count (such as transitivity) can be sensitive to these variations in graph size, causing errors in the anomaly detection process.

We refer to statistics that vary with network size as Size Inconsistent-more or fewer messages/ nodes change the output of the statistic, even when the underlying property that the statistic is intended to measure is constant, making graph size a confounding factor.

When a statistic is affected by a confounding factor like communication volume, any anomaly detector that employs that statistic is susceptible to producing false positives or false negatives. False positives are times reported as anomalies when they are in fact examples of normal behavior; false negatives are anomalous times that were not identified as anomalies. Confounding factors are capable of producing both types of errors when testing using an affected statistic: false positives result from a detection triggered by changes in the confounding factor rather than the network property intended to be measured; false negatives are caused by noise from a confounding factor drowning out the signal of a true anomaly.

Even on an Erdös-Rényi network, which does not explicitly capture transitive relationships through a network property, statistics like the clustering coefficient are greater as the number of edges in the network increases, since more triangles are closed due to random chance. In other words, the clustering coefficient is sensitive to changes in the communication volume when it is intended to measure the transitivity property independent of communication volume.

Figure 1 shows the effect of this Size Inconsistency when detecting anomalies in real data. The figure shows data from the Enron e-mail corpus, with the vertical lines representing times where major events occurred in the network. Figure 1(a) shows the total message count per week in the network, whereas Figure 1(b) shows the Graph Edit Distance (GED) at each week. Green circles indicate correctly identified anomalies, whereas red circles are false positives. GED is a Size Inconsistent statistic and therefore correlates strongly with the total message count. This causes it to produce many false positives, and the anomalies that it does correctly identify could have been found by analyzing the message count.

The solution to this issue is to use Size Consistent statistics: statistics that produce values that are not sensitive to variations in the graph size, but depend only on the underlying properties of the graph they were intended to measure. Figure 1(c) shows Probability Mass Shift, a Size Consistent statistic, when applied to the Enron e-mail corpus. Unlike Edit Distance, the Probability Mass Shift is insensitive to variations in the total message and node count and is able to detect more of the anomalous events. By utilizing the assumptions of the data model described in Section 2, 


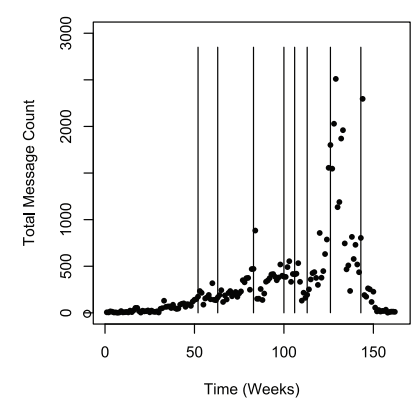

(a)

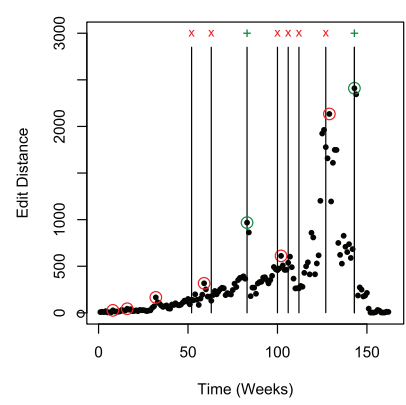

(b)

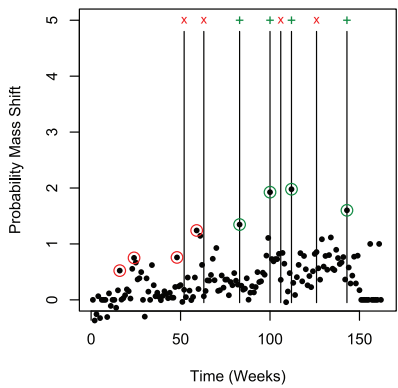

(c)

Fig. 1. Enron e-mail data corpus with the dates of several major events highlighted. Green pluses and circles indicate correctly identified anomalies. Red circles indicate false positives and red X's false negatives. (a) Total messages sent at each time step. (b) Anomalies detected by Graph Edit Distance statistic. (c) Anomalies detected by Probability Mass Shift statistic.

we develop a framework for developing new Size Consistent statistics which are insensitive to the effect of message and node count. In Section 4, we design several Size Consistent statistics such as Probability Mass Shift using this framework, which are Size Consistent counterparts to standard network statistics. Theory describing the properties of Size Consistent and Inconsistent statistics can be found in Section 3.1.

In Section 4, we examine many commonly used network statistics and prove that most of them are Size Inconsistent, suffering the problems inherent to Size Inconsistent statistics as proven in the theory. To alleviate these issues, we establish a framework for defining new statistics that are Size Consistent. We then use this framework to develop three new Size Consistent statistics: Probability Mass Shift, Probabilistic Degree Distance, and Triangle Probability (TP). These statistics have parallels to existing network statistics but have superior anomaly detection performance due to their status as Size Consistent statistics.

In addition to identifying theoretical consistency properties of network statistics, we verify the effect of these properties through synthetic, semi-synthetic, and real-data experiments. The anomaly detection algorithm used is described in Section 5, while the experiments themselves are in Section 6. The synthetic and semi-synthetic experiments validate the utility of Size Consistent statistics by determining their ability to recover inserted anomalies in either a synthetic or real-data background. The real-data experiments apply the anomaly detector to real networks and report the types of anomalies discovered. Section 7 analyzes the detected anomalies in detail, showing how decomposing the anomaly score can give greater insight into the nature of detected anomalies.

The major contributions of this work are as follows:

- We define Size Inconsistent and Size Consistent properties of network statistics and show that Size Consistent statistics have fewer false positives and false negatives than Inconsistent statistics.

- We prove that several commonly used network statistics are Size Inconsistent and fail to capture the network behavior with varying network size.

- We introduce a framework for developing new statistics that are provably Size Consistent and insensitive to variations in the message or node count.

- Using our framework, we introduce several provably Size Consistent statistics that are comparable to commonly used Size Inconsistent statistics. 
Table 1. Glossary of Terms

\begin{tabular}{|c|c|}
\hline$G_{t}$ & Observed graph at time $t$ \\
\hline$V_{t}$ & Set of vertices in graph $G_{t}$, size $N$ \\
\hline$W_{t}$ & Weighted adjacency matrix of $G_{t}$ \\
\hline$\left|W_{t}\right|$ & Total weight of $W_{t}$ \\
\hline$P_{t}^{*}$ & True distribution of edge weights in the underlying model, size $\left|V^{*}\right| \times N^{*}$ \\
\hline$A_{t}^{*}$ & Adjacency matrix of $P_{t}^{*}$; i.e., $a_{i j, t}=I\left[p_{i j, t}^{*}>0\right]$ \\
\hline$V^{*}$ & True vertex set of underlying model, $V_{t} \subset V^{*}$ \\
\hline$P_{t}$ & Renormalized distribution of edge weight on vertex set $V_{t}$, used to sample $G_{t}$ \\
\hline$A_{t}$ & Adjacency matrix of $P_{t}$; i.e., $a_{i j, t}=I\left[p_{i j, t}>0\right]$ \\
\hline$\left|A_{t}\right|$ & Number of non-zero cells in adjacency matrix \\
\hline$\widehat{P}_{t}$ & Approximate distribution of edge weights estimated from $G_{t}: \widehat{p}_{i j, t}=\frac{w_{i j, t}}{\left|W_{t}\right|_{1}}$ \\
\hline$\widehat{A}_{t}$ & Adjacency matrix of $G_{t}$; i.e., $a_{i j, t}=I\left[w_{i j, t}>0\right]$ \\
\hline$\overline{p^{*}}{ }_{t}$ & Mean value of any non-zero cell in $P_{t}^{*}$ \\
\hline $\bar{p}_{t}$ & Mean value of any non-zero cell in $P_{t}$ \\
\hline & Mean value of any non-zero cell in $\widehat{P}_{t}$ \\
\hline$\overline{p^{*}}{ }_{t} \mid V_{t}$ & Mean value of the $P_{t}^{*}$ cells that belong to vertex subset $V_{t}$ \\
\hline$w_{\text {row }_{i}, 2}$ & Total weight in row $i$ of $W_{t}$ \\
\hline$\overline{p^{*}}$ row, $t$ & Expected mass in any row of $P_{t}^{*}$ \\
\hline $\bar{p}_{\text {row }, t}$ & Expected mass in any row of $P_{t}$ \\
\hline$\overline{\bar{p}}_{\text {row }, t}$ & Expected mass in any row of $\widehat{P}_{t}$ \\
\hline${\overline{p^{*}}}_{\text {row }, t} \mid V_{t}$ & Expected mass of rows in $P_{t}^{*}$, excluding any rows or cells that do not belong to $V_{t}$ \\
\hline
\end{tabular}

- In synthetic and semi-synthetic experiments, we demonstrate that our proposed statistics have superior precision and recall compared to conventional statistics when detecting inserted network anomalies.

- We demonstrate that in real-world data our proposed statistics detect more interesting anomalies than alternatives, and show using local anomaly decomposition that the detected anomalies correspond to distinct events occurring in the networks.

\section{PROBLEM DEFINITION AND DATA MODEL}

In order to formulate theory regarding detection of network anomalies, we must make some assumptions about how networks form and evolve over time. We codify these assumptions using a general statistical model of graph generation, expanded to accommodate graphs of varying size and density. We describe the model in detail below and follow that with a formal definition of the anomaly detection problem.

The dynamic network model must produce a sequence of graph structures and must allow for varying numbers of edges and nodes in those graphs. Once we have established the basic graph construction procedure, we can define what it means for the graph to behave normally versus what it means to be anomalous.

Table 1 shows a glossary of terms that are used throughout this article.

Let $G=\{V, W\}$ be a weighted graph that represents a network, where $V$ is the node set and $W$ is the weighted adjacency matrix representing messages or some other interaction, with $w_{i j}$ the number of messages between nodes $i$ and $j$. Let $|V|$ and $|W|$ refer to the number of nodes, and total weight of the edges, in $G$ respectively. A dynamic network is simply a set of graphs $\left\{G_{1}, G_{2}, \ldots G_{T}\right\}$ 
where each graph represents network activity within a consistent-width time step (e.g., one step per day).

To accommodate the observations of graphs of varying size, let us assume the models that generated the observed graphs are hidden but take the form of a multinomial sampling procedure. Let $P^{*}$ be a $\left|V^{*}\right| \times\left|V^{*}\right|$ matrix where the rows and columns represent a node set $V^{*}$ and the sum of all cells is equal to 1 . Here, $V^{*}$ represents a large set of possible nodes, i.e., larger than the set we may see in any one graph $G$. The entry $p_{i j, t}^{*}$ specifies the probability that a randomly sampled message at time $t$ is between $i$ and $j$. Let $|V|$ and $|W|$ be drawn from distributions $M_{V}$ and $M_{W}$.

The full generative process for all graphs is then

-Draw $|V| \sim M_{V}$. Select $V$ from $V^{*}$ uniformly at random.

- Construct $P$ by selecting the rows/columns from $P^{*}$ that correspond to $V$ and normalize the probabilities to sum to 1 (i.e., $p_{i j}=\frac{1}{Z} p_{i j}^{*}$, where $Z=\sum_{i j \in V} p_{i j}^{*}$ ).

-Draw $|W| \sim M_{W}$. Sample $|W|$ messages using probabilities $P$.

-Construct the graph $G=(V, W)$ from the sampled messages.

$G$ is the output of a multinomial sampling procedure on $P$, with each independent message sample increasing the weight of one cell in $W . P$ itself is a set of probabilities obtained by sampling $V$ from $V^{*}$. This graph generation process is summarized in Figure 3.

Note that this basic data model generalizes a class of commonly used graph models such as Stochastic Block Models, the Chung-Lu model [1], and KPGM [32], and any other model that defines a Bernoulli edge observation probability $p_{i j}$. The difference between our data model and these others is that (1) our model accommodates for variation in the number of nodes through the node sampling step, while other models typically assume a constant number of nodes, and (2) our model requires that $P^{*}$ and $P$ are proper probability distributions and must sum to 1 allowing for sampling from the space of all possible edges.

Now that we have defined how the observed data is created, we can now define the task of anomaly detection on this data:

Problem definition: Given a stream of graph examples $\left\{G_{1}, G_{2}, \ldots, G_{t-1}\right\}$ drawn from a normal model $M^{n}$, and a graph $G_{t}$ drawn from an unknown model, determine if $G_{t}$ was drawn from $M^{n}$ or some anomalous model $M^{a}$.

Given an observed graph, we wish to decide if this graph exhibits the same behavior (network properties) as past graph examples or if is likely the product of some different, anomalous process. We solve this problem with hypothesis tests, utilizing network statistics as the test statistics. If $S_{k}(G)$ is some network statistic designed to measure a network property $k$, then the set of statistics calculated on the normal examples $\left\{S_{k}\left(G_{1}\right), S_{k}\left(G_{2}\right), \ldots, S_{k}\left(G_{t-1)}\right\}\right.$ forms the empirical null distribution, and the value $S_{k}\left(G_{t}\right)$ is the test point. The anomaly detection procedure is summarized in Figure 2 from model down to null distribution and test point.

For this work, we use a two-tailed test with $p$-values of $\alpha=0.05$. Anomalous test cases where the null hypothesis is rejected correspond to true positives; normal cases where the null hypothesis is rejected correspond to false positives. Likewise anomalous cases where the null is not rejected correspond to false negatives and normal cases where the null is not rejected correspond to true negatives.

If all the graph examples have the same number of edges and nodes then graph size cannot be a confounding factor regardless of the choice of test statistic-those properties are naturally controlled in the data. However, if $M^{n}$ and $M^{a}$ produce graphs with a variable number of edges and nodes then any test statistic needs to be robust to changes in the graph size. Ideally, if $G_{x}, G_{y} \sim M$ but $\left|V_{x}\right| \neq\left|V_{y}\right|,\left|W_{x}\right| \neq\left|W_{y}\right|$, we would still want $S_{k}\left(G_{x}\right) \approx S_{k}\left(G_{y}\right)$ to be true. 


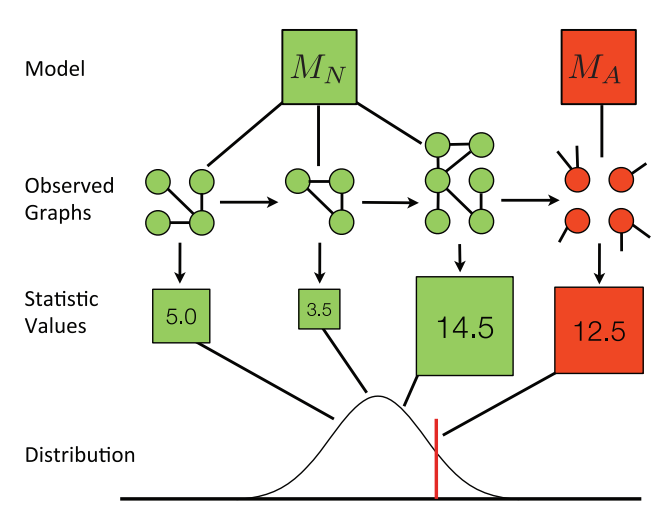

Fig. 2. Dynamic network anomaly detection task. Given past instances of graphs created by the typical model of behavior, identify any new graph instance created by an alternative anomalous model.

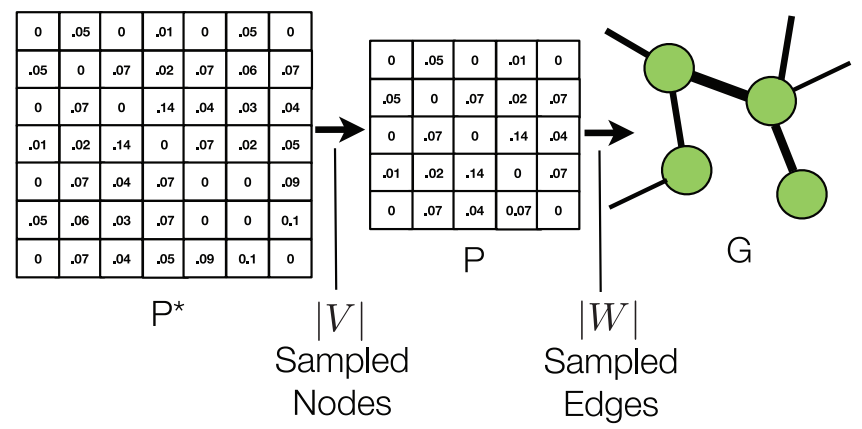

Fig. 3. Graph generation process. The matrix $P^{*}$ represents all possible nodes and their interaction probabilities. By sampling $|V|$ nodes and $|W|$ edges the observed graph $G$ is obtained.

Given this generative process, the difference between normal and anomalous graphs is characterized by differences in their underlying models. Let the normal model be represented by $P^{* n}, M_{V}^{n}$, and $M_{W}^{n}$ and let the anomalous model be represented by $P^{* a}, M_{V}^{a}$, and $M_{W}^{a}$. Finding instances where $M_{V}$ or $M_{W}$ are anomalous is trivial since we can use the count of nodes or messages as the test statistic. Finding instances of graphs drawn from $P^{* a}$ is more difficult as our choice of network statistics affects whether we are sensitive to changes in $|W|$ or $|V|$.

If we redefine our network statistics to be functions over $P^{*}$ instead of $G$, we avoid the problem of graph size as a confounding factor as $P^{*}$ is independent of $M_{V}$ or $M_{W}$. However, since $P^{*}$ is unobservable, there is no way to calculate $S_{k}\left(P^{*}\right)$ directly. Instead we can only calculate $\widehat{S}_{k}(G)$ from the observed graph $G . \widehat{S}_{k}(G)$ is an estimate of $S_{k}(P)$ using the sampled messages $W$ to estimate the underlying probabilities, and $S_{k}(P)$ itself is an estimate of the true $S_{k}\left(P^{*}\right)$ on a subset $V$ of the total nodes. So just as the sampling procedure follows $P^{*} \rightarrow P \rightarrow G$, the estimation procedure follows the inverse steps $\widehat{S}_{k}(G) \rightarrow S_{k}(P) \rightarrow S_{k}\left(P^{*}\right)$.

Delta statistics, which measure the difference between two networks (e.g., GED), can also be used for anomaly detection. In this case, the empirical statistic is $\widehat{S}_{k}\left(G_{1}, G_{2}\right)$, where $G_{1}$ and $G_{2}$ are generated using the graph generation procedure described above, and the true value of the statistic is $S_{k}\left(P_{1}^{*}, P_{2}^{*}\right)$. In order to be consistent, Delta statistics should not change when either graph changes in size. 
Ideally, $\widehat{S}_{k}(G)=S_{k}(P)=S_{k}\left(P^{*}\right)$ and we would have the same output regardless of $W$ and $V$, being sensitive only to changes in the model. However, this is typically not attainable in practice as it is difficult to estimate the true statistic value from graphs that are extremely small-few edges and nodes provides less evidence of the underlying properties. In addition, an unbiased statistic with extremely high variance is also a poor test statistic. In many scenarios, the best statistics are ones that converge to the value of $S_{k}\left(P^{*}\right)$ as $|V|,|W|$ increase, a property that we refer to as Size Consistency.

The above definition of the data generation and the task of detecting anomalous graphs generated by the model will become the foundations of the theory regarding the consistency or inconsistency of network statistics and their utility in detecting anomalies. In the next section, we formally define the properties of Size Consistent and Size Inconsistent statistics and show how they affect the accuracy of hypothesis tests.

\section{PROPERTIES OF THE TEST STATISTIC}

\subsection{Size Consistency}

As described previously, a statistic $S_{k}\left(P^{*}\right)$ depends on the properties of the procedure that generated the graph instance and is a measure of the graph properties independent of the exact number of edges and nodes in the graph. Although the empirical statistic $\widehat{S}_{k}(G)$ may not be independent of the edge and node count on smaller graph samples, if it converges to $S_{k}\left(P^{*}\right)$ as $|V|$ and $|W|$ increase then the effect of edge and node count on the empirical statistic diminish until they are negligible.

Definition 3.1. A statistic $\widehat{S}_{k}$ is Size Consistent if and only if a function $S_{k}$ exists such that

$$
\lim _{|W| \rightarrow \infty} \widehat{S}_{k}(G)=S_{k}(P) \quad \text { AND } \quad \lim _{|V| \rightarrow\left|V^{*}\right|} S_{k}(P)=S_{k}\left(P^{*}\right),
$$

where $S_{k}$ is defined and finite for all input $P$-matices.

Delta statistics have the same requirements for consistency as singular graph statistics except that they have an edge count and node count term for each of the input graphs. This requires that we show consistency for multivariate limits rather than single limits:

Definition 3.2. A delta statistic $\widehat{S}_{k}$ is Size Consistent if and only if a function $S_{k}$ exists such that $\lim _{\left(\left|W_{1}\right|,\left|W_{2}\right|\right) \rightarrow(\infty, \infty)} \widehat{S}_{k}\left(G_{1}, G_{2}\right)=S_{k}\left(P_{1}, P_{2}\right) \quad$ AND $\quad \lim _{\left(\left|V_{1}\right|,\left|V_{2}\right|\right) \rightarrow\left(\left|V^{*}\right|,\left|V^{*}\right|\right)} S_{k}\left(P_{1}, P_{2}\right)=S_{k}\left(P_{1}^{*}, P_{2}^{*}\right)$, where a single $S_{k}$ exists and is finite for all input $P$-matrix pairs.

Unlike the single statistic case, there is another way for a delta statistic to fail the requirements for Size Consistency: the multivariate limit might not exist. In order for a multivariate limit to exist there must be a single value that the function approaches regardless of the direction of the approach. If $\widehat{S}_{k}\left(G_{1}, G_{2}\right)$ or $S_{k}\left(P_{1}, P_{2}\right)$ can converge to two different values as $\lim _{\left(\left|W_{1}\right|,\left|W_{2}\right|\right) \rightarrow(\infty, \infty)}$ or $\lim _{\left(\left|V_{1}\right|,\left|V_{2}\right|\right) \rightarrow\left(\left|V^{*}\right|,\left|V^{*}\right|\right)}$ then the statistic is not Size Consistent even if those values are both finite. We refer to these Size Inconsistent cases as "no multivariate limit" scenarios. A simple method to prove Size Inconsistency for delta statistics is to find two approaches to the limit (e.g., $\left|W_{1}\right|=\left|W_{2}\right|$ versus $\left.\left|W_{1}\right|=\left|W_{2}\right|^{2}\right)$ that converge to two different values; if two such approaches can be found then the statistic is Size Inconsistent, but if all approaches converge to the same finite value then the statistic is Size Consistent.

A simple approach to evaluating the Size Consistency of a delta statistic is to reduce it to a limit over one variable. In this case if the limit is finite then the statistic is Size Consistent. One way to do this is to use the properties of limits over algebraic functions to simplify a multivariate limit: 
Lemma 3.1. If $\lim _{(a) \rightarrow\left(a_{0}\right)} F_{1}(a)$ has a limit finite limit $L_{1}$ for all values of $a_{0}$ and $\lim _{(b) \rightarrow\left(b_{0}\right)} F_{2}(b)$ has a limit finite limit $L_{2}$ for all values of $b_{0}$, then for an algebraic function $H$, $\lim _{(a, b) \rightarrow\left(a_{0}, b_{0}\right)} H\left[F_{1}(a), F_{2}(b)\right]$ has a finite limit equal to $H\left[L_{1}, L_{2}\right]$ for all values of $a_{0}, b_{0}$.

Proof. This can be shown using the basic properties of limits. The limit over the sum, difference, multiple, square, or root of function(s) is the operator applied to their limit(s), so

$$
\lim _{(a, b) \rightarrow\left(a_{0}, b_{0}\right)} H\left[F_{1}(a), F_{2}(b)\right]=H\left[\lim _{(a, b) \rightarrow\left(a_{0}, b_{0}\right)} F_{1}(a), \lim _{(a, b) \rightarrow\left(a_{0}, b_{0}\right)} F_{2}(b)\right] .
$$

These can be decomposed into single limits, giving us a solution of $H\left[L_{1}, L_{2}\right]$. If both $L_{1}$ and $L_{2}$ exist and are finite values, this equation exists and is finite as well.

So to evaluate the existence and finiteness of a dual limit that follows this form, we need only evaluate the existence and finiteness of the limits over the functions that are its inputs. In many cases these input functions involve only a single variable, which lets us reduce the dual limit to a single limit.

The utility of Size Consistency for graph statistics lies in the reduction of errors in hypothesis tests made using consistent statistics. As the statistics approach their limits, the effect of changes in the edge or node count diminishes to zero, and so the probability of producing an error due to variation in the edge or node count also approaches zero. This affects both the false positive and false negative rate:

Theorem 3.1 [False Positive Rate for Size Consistent Statistics]. Let $\left\{G_{1} \ldots G_{x}\right\}$ be a finite set of "normal" graphs drawn from $P^{*}, M_{W}^{n}$, and $M_{V}^{n}$ and let $G_{\text {test }}$ be a test graph drawn from $P^{*}$, $M_{W}^{a}$, and $M_{V}^{a}$. Let $\left|W_{\text {min }}\right|$ be the minimum edge count in both $\left\{G_{x}\right\}$ and $G_{\text {test }}$ and $\left|V_{\text {min }}\right|$ be the minimum node count. For a hypothesis test using a Size Consistent test statistic $\widehat{S}_{k}$ and a p-value of $\alpha$, as $\left|W_{\text {min }}\right| \rightarrow \infty$ and $\left|V_{\text {min }}\right| \rightarrow\left|V^{*}\right|$ the probability of identifying $G_{\text {test }}$ as a false positive approaches $\alpha$.

The proof of Theorem 3.4 is left to the appendix. As the number of edges and nodes drawn for the null distribution and test instance increase, the bias $a b s\left(S_{k}\left(P^{*}\right)-\widehat{S}_{k}(G)\right)$ of the statistic calculated on those networks converges to zero. This means that $\widehat{S}_{k}(G)$ effectively becomes equal to $S_{k}\left(P^{*}\right)$, and the outcome of the hypothesis test is only dependent on whether the test instance and null examples were both drawn from $P^{* n}$ or if the test instance was drawn from $P^{* a}$. Even if the test case has an abnormal number of edges or nodes, as long as the minimum number of edges and nodes in both the test and null graphs is above some threshold there will not be a false positive due to graph size.

Now, we examine the false negative rate:

Theorem 3.2 [False Negative Rate for Size Consistent Statistics]. Let $\left\{G_{1} \ldots G_{x}\right\}$ be a finite set of "normal" graphs drawn from $P^{n *}, M_{W}^{n}$, and $M_{V}^{n}$ and let $G_{\text {test }}$ be a test graph drawn from $P^{a} *, M_{W}^{a}$, and $M_{V}^{a}$ that is anomalous with respect to property $k$. Let $\left|W_{\text {min }}\right|$ be the minimum of $M_{W}^{n}$ and $\left|V_{\text {min }}\right|$ be the minimum of $M_{V}^{n}$. For a hypothesis test using a Size Consistent test statistic $\widehat{S}_{k}$, which converges to $S_{k}$ as $\left|W_{\text {min }}\right| \rightarrow \infty$ and $\left|V_{\text {min }}\right| \rightarrow\left|V^{*}\right|$ the probability of failing to reject $G_{\text {test }}$ in the limit of $\left|W_{\text {min }}\right|$ and $\left|V_{\text {min }}\right|$ approaches 0 if $S_{k}\left(P^{* n}\right) \neq S_{k}\left(P^{* a}\right)$.

See the appendix for proof. Similar to the false positive case, there is no deviation from the true limit if the number of observed edges and nodes is sufficiently large. If the test graph and the null graphs are drawn from different underlying models and $S_{k}$ is capable of distinguishing those models, then a hypothesis test using $\widehat{S}_{k}$ will also distinguish them if $\left|W_{\text {min }}\right|$ and $\left|V_{\text {min }}\right|$ are sufficiently large. 
Note that while Size Consistency is a necessary property for a good test statistic, it is not a sufficient condition and less useful Size Consistent statistics exist. Consider statistics that are linear in terms of the node count $|V|$. As Size Consistency only requires that a finite limit exist w.r.t. $|V|$ these statistics fulfill that requirement; however, they approach that limit diagonally and the error will be proportional to the number of unobserved nodes in the population (i.e., $\left.\left|V^{*}\right|-|V|\right)$. Such statistics will only achieve their limit at the point $|V|=\left|V^{*}\right|$ and for practical purposes behave as if they were diverging. We will designate these statistics as having a Non-Constant Error Bound and define them to be any statistic that has a max error bound, which is greater than $O(1)$ in the number of nodes. We will also refer to these statistics as Pseudo-Diverging, as in some circumstances the statistics behave as if they were diverging to infinity.

Definition 3.3. A statistic $\widehat{S}_{k}$ is Pseudo-Diverging if it is Size Consistent and has a Non-Constant Error Bound $\epsilon_{\max }$ that is greater than $O(1)$ w.r.t. $|V|$, where $\epsilon_{\max }$ is an upper bound on the bias of the empirical statistic: $\operatorname{abs}\left(S_{k}\left(P^{*}\right)-\widehat{S}_{k}(G)\right) \leq \epsilon_{\max }$.

The converse is a statistic that has a Constant Error Bound, simply defined as any statistic with an error bound that is $O(1) . O(1)$ refers to the big-O notation of constancy; any function that can be bounded by a constant $k$ rather than a function of $|V|$ will have a Constant Error Bound. This is much preferable as a test statistic as the statistic can be closer to its limit without having seen a majority of the node population. Typically the probability of a statistic reaching the maximum error bound also decreases as $|V|$ increases. Note that the issue of Non-Constant versus Constant Error Bounds arises because the limit of $|V|$ is a constant in our data model: As $W$ is an infinite limit, we do not encounter Pseudo-Divergency with respect to edges.

Now that we have investigated the effects of Size Consistency on hypothesis test accuracy, we must look at the effects of Size Inconsistency.

\subsection{Size Inconsistency}

Size Inconsistency is the inverse of Size Consistency: if a statistic is not Size Consistent, then it is size inconsistent.

Definition 3.4. A statistic $\widehat{S}_{k}$ is Size Inconsistent if no function $S_{k}$ exists such that $\widehat{S}_{k}(G)$ is Size Consistent with a limit of $S_{k}\left(P^{*}\right)$, where $S_{k}$ is defined and finite for all $P^{*}$.

We refer to any statistic that is Size Inconsistent due to having an infinite $S_{k}$ as Size Divergent.

Delta statistics can also be Size Divergent if they have an infinite limit. However, they can also fail the requirements for Size Consistency if they fall into the no mulitvariate limit scenario described earlier by having no single multivariate limit. Both Size Divergence and no multivariate limit can cause a statistic to produce false positives/negatives due to the size of the sampled networks:

Theorem 3.3 [False Positives for Size Divergent Statistics]. Let $\left\{G_{1} \ldots G_{x}\right\}$ be a finite set of "normal" graphs drawn from $P^{*}, M_{W}^{n}$, and $M_{V}^{n}$ where $M_{W}^{n}$ and $M_{V}^{n}$ have finite bounds. Let $G_{\text {test }}$ be a test graph also drawn from $P^{*}$ with size $\left|W_{\text {test }}\right|$, $\left|V_{\text {test }}\right|$. If $\widehat{S}_{k}(G)$ diverges with increasing $|W|$ or $|V|$, there is some $\left|W_{\text {test }}\right|$ or $\left|V_{\text {test }}\right|$ for which a hypothesis test using $\widehat{S}_{k}(G)$ as the test statistic will incorrectly flag $G_{\text {test }}$ as an anomaly.

See the appendix for proof. If a statistic is Size Inconsistent then it is dependent on the size of a graph, and two graphs both drawn from $P^{* n}$ may produce entirely different values if their edge/node counts differ, producing a false positive.

A second problem occurs when the edge counts in the estimation set have high variance. If the statistic is dependent on the number of edges, noise in the edge counts translates to noise in the 
statistic values that lowers the statistical power (i.e., the percentage of true anomalies detected) of the test. With a sufficient amount of edge count noise, the signal is completely drowned out and the statistical power of the anomaly detector drops to zero.

Theorem 3.4 [False Negatives for Size Divergent Statistics]. Let $\left\{G_{1} \ldots G_{x}\right\}$ be a finite set of "normal" graphs drawn from $P^{*}, M_{W}^{n}$, and $M_{V}^{n}$ where $M_{W}^{n}$ and $M_{V}^{n}$ have finite bounds. Let $G_{\text {test }}$ be a network that is anomalous (i.e., drawn from $P^{* a}, M_{W}^{n}$, and $M_{V}^{n}$ ) with respect to property $k$. If $\widehat{S}_{k}(G)$ diverges with increasing $|W|$ or $|V|$ there exists some $M_{W}^{n}$ or $M_{V}^{n}$ with sufficient variance such that a hypothesis test with p-value $\alpha$ and empirical null distribution $\widehat{S_{k}}$ will fail to detect $S_{k}\left(G_{t e s t}\right)$ as an anomaly with probability $1-\alpha$.

See the appendix for proof. With a sufficient amount of edge count noise, the statistical power of the anomaly detector drops to zero. Regardless of whether a time step is an example of an anomaly or not, if the variance of the test statistic is dominated by random noise in the edge count the time step will only be flagged due to random chance.

Now, we examine the behavior of statistics with no Multivariate Limit:

Theorem 3.5 [False Positives for Statistics with No Multivariate Limit]. Let $\left\{G_{1} \ldots G_{d}\right\}$ be a finite set of "normal" graphs drawn from $P^{*}, M_{W}^{n}, M_{V}^{n}$ and let $G_{\text {test }, 1}, G_{\text {test }, 2}$ be a pair of test graphs drawn from $P^{*}, M_{W}^{a}, M_{V}^{a}$. Let $\left|W_{\text {min }}\right|$ be the minimum number of edges in all graphs and $\left|V_{\text {min }}\right|$ be the minimum node count. If $\widehat{S}_{k}\left(G_{x}, G_{y}\right)$ converges to $S_{1}$ from direction $A$ and converges to $S_{2}$ from direction $B, S_{1} \neq S_{2}$, where $A$ and $B$ are different approaches to the dual limits $\left|W_{x}\right|,\left|W_{y}\right| \rightarrow \infty, \infty$ and $\left|V_{x}\right|,\left|V_{y}\right| \rightarrow\left|V^{*}\right|,\left|V^{*}\right|$, then there is some $M_{W}^{n}, M_{V}^{n}, M_{W}^{a}, M_{V}^{a}$ for which a hypothesis test will incorrectly flag $G_{\text {test }, 1}, G_{\text {test }, 2}$ as an anomaly as $\left|W_{\text {min }}\right| \rightarrow \infty$ and $\left|V_{\text {min }}\right| \rightarrow V^{*}$.

See the appendix for proof. If multiple values can be produced by the same statistic based on the direction of the approach to the limit then there is nothing preventing the test statistic and the null statistics from approaching the same limit in different directions and producing a false positive. False negatives can also be produced due to having no multivariate limit:

Theorem 3.6 [False Negatives for Statistics with No Multivariate Limit]. Let $\left\{G_{1} \ldots G_{X}\right\}$ be a finite set of "normal" graphs drawn from $P^{* n}, M_{W}^{n}$, and $M_{V}^{n}$. Let $G_{\text {test }}$ be a network that is anomalous (i.e., drawn from $P^{* a}, M_{W}^{n}$, and $M_{V}^{n}$ ) with respect to propertyk. Let $\left|W_{\text {min }}\right|$ be the minimum number of edges in all graphs and $\left|V_{\text {min }}\right|$ be the minimum node count. If $\widehat{S}_{k}$ converges to either $S_{\text {lower }}^{n}$ or $S_{u p p e r}^{n}$ on pairs of graphs drawn from $P^{* n}$ depending on the approach or $S^{a}$ on when one of the graphs was drawn from $P^{* a}, S_{\text {lower }}^{n}<S^{a}<S_{u p p e r}^{n}$, then there exists some $M_{W}^{n}, M_{V}^{n}$ such that as $\left|W_{\text {min }}\right| \rightarrow \infty$ and $\left|V_{\text {min }}\right| \rightarrow\left|V^{*}\right|$ a hypothesis test using $\widehat{S}_{k}$ as a test statistic and a p-value of $\alpha$ will produce a false negative with probability 1 .

See the appendix for proof. Inconsistent statistics have no guarantees in terms of false positives and negatives caused by graph size while consistent statistics reduce the chance of error as the size of the graphs grows. Therefore, it is beneficial to use a Size Consistent statistic in place of a similar but inconsistent statistic. In the next section, we show that many common network statistics are inconsistent, and we introduce Size Consistent alternatives to those statistics.

\subsection{Procedure for Determining a Statistic's Properties}

Now that we have defined the possible characteristics for network statistics, determining which characteristics apply to a given statistic is a straightforward process of applying three tests sequentially. This process is outlined in Figure 4. First, the statistic must not have an infinite limit; if an infinite limit exists it is Size Inconsistent and Diverging. Second, the statistic must approach 


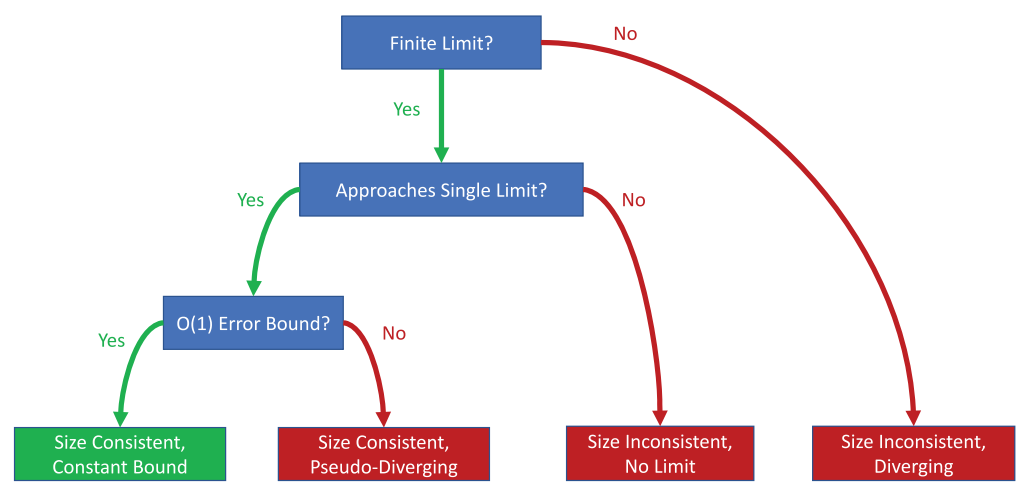

Fig. 4. Decision process for determining a statistic's characteristics. A statistic must have a single, finite limit and have an $O(1)$ error bound in order to be Size Consistent with a Constant Error Bound.

Table 2. Consistency Properties of Previous Network Statistics and Our Proposed Alternatives

\begin{tabular}{l|c|c|c|c|}
\hline & \multicolumn{2}{|c|}{ Theoretical properties } & \multicolumn{2}{c|}{ Robust performance } \\
& Size consistent & Error bound & w.r.t. edge variation & w.r.t. node variation \\
\hline Mass shift & $\checkmark$ & $O(1)$ & $\checkmark$ & $\checkmark$ \\
Probabilistic degree & $\checkmark$ & $O(1)$ & $\checkmark$ & $\checkmark$ \\
Triangle probability & $\checkmark$ & $O(1)$ & $\checkmark$ & X \\
\hline Graph edit distance & $\mathrm{X}$ & N/A & $\mathrm{X}$ & X \\
Degree distribution & $\mathrm{X}$ & N/A & $\mathrm{X}$ & $\mathrm{X}$ \\
Barrat clustering & $\checkmark$ & $O(1)$ & $\checkmark$ & X \\
Unweighted netsimile & $\checkmark$ & $O(1)$ & $\checkmark$ & X \\
Weighted netsimile & $\mathrm{X}$ & N/A & X & X \\
Deltacon & $\checkmark$ & $O(|V|)$ & X & \\
\hline
\end{tabular}

a single limit as $|W| \rightarrow \infty$ and $|V| \rightarrow\left|V^{*}\right|$ from any direction; if it does not it is Size Inconsistent with No Multivariate Limit. If the statistic passes these tests, it is Size Consistent and the only remaining question is the order of its error bound. If an error bound of $O(1)$ exists the statistic is Size Consistent with a Constant Error Bound, otherwise it is Size Consistent but is Pseudo-Diverging.

\section{NETWORK STATISTICS}

In this section, we introduce our set of proposed Size Consistent statistics, as well as analyze multiple previously defined conventional statistics to determine their consistency properties. These properties are summarized in Table 2; Size Consistent refers to statistics, which meet the requirements for theoretical Size Consistency, while Robust Performance refers to the low False Positive and False Negative rates when there is noise from variation in edge count (Edge Change) or node count (Node Change) as determined in semi-synthetic experiments in Section 6. For statistics that are Size Consistent, the big-O complexity of the error bound is also provided. Note that Size Consistency and a Constant Error Bound are not sufficient to ensure empirical performance, as empirical performance requires quick convergence to the limit on data with properties appropriate for real-world networks. 


\subsection{Conventional Statistics}

Graph edit distance. The GED [22] is often used in anomaly detection tasks. GED on a weighted graph is typically defined as

$$
\operatorname{GED}\left(G_{1}, G_{2}\right)=\left|V_{1}\right|+\left|V_{2}\right|-2\left|V_{1} \cap V_{2}\right|+\sum_{i j \in V_{1} \cup V_{2}} a b s\left(w_{i j, 1}-w_{i j, 2}\right) .
$$

Claim 4.1. GED is a Size Inconsistent statistic.

See the appendix for proof.

Degree distribution and degree distribution difference. The Degree Distribution of a graph is simply the distribution of the individual node degrees. To capture the difference between the degree distributions of two graphs, we define a delta statistic, Degree Distribution Difference, as

$$
D D\left(G_{1}, G_{2}\right)=\sum_{d \in\left[1, d_{\text {max }}\right]} a b s\left(\sum_{i \in V_{1}} I\left[D_{i}\left(G_{1}\right)=d\right]-I\left[\sum_{i \in V_{2}} D_{i}\left(G_{2}\right)=d\right]\right),
$$

where $D_{i}\left(G_{x}\right)$ is the weighted degree of node $i$ in graph $G_{x}$ and $d_{\text {max }}$ is the largest degree in $G_{1}$ or $G_{2}$. The weighted degree can be expressed as $\sum_{j \in V_{x}} w_{i j, x}$.

Claim 4.2. Degree Distribution Difference is a Size Inconsistent statistic.

See the appendix for proof. Other measures create aggregates using the degrees of multiple nodes $[7,41]$ but as the degree distribution is Size Inconsistent these aggregates are as well.

Weighted clustering coefficient. Clustering coefficient is a measure of the transitivity, the propensity to form triangular relationships in a network. As the standard clustering coefficient is not designed for weighted graphs analyze a weighted clustering coefficient, specifically the Barrat weighted clustering coefficient $(\mathrm{CB})[43]$ :

$$
C B(G)=\sum_{i} \frac{1}{|V|\left(a_{i}-1\right) D_{i}(G)} \sum_{j, k} \frac{w_{i j}+w_{i k}}{2} \widehat{a}_{i j} \widehat{a}_{i k} \widehat{a}_{j k}
$$

where $\widehat{a}_{i j}=I\left[w_{i j}>0\right], D_{i}(G)=\sum_{j} w_{i j}$, and $a_{i}=\sum_{j} a_{i j}$. Other weighted clustering coefficients exist but they behave similarly to the Barrat coefficient.

Claim 4.3. Barrat Clustering is a Size Consistent statistic that converges to

$$
C B\left(P^{*}\right)=\sum_{i} \frac{1}{\left|V^{*}\right|\left(a_{i}^{*}-1\right) \sum_{j} p_{i j}^{*}} \sum_{j, k} \frac{\left(p_{i j}^{*}+p_{i k}^{*}\right)}{2} a_{i j}^{*} a_{i k}^{*} a_{j k}^{*} .
$$

See the appendix for proof. Other weighted clustering coefficients such as those proposed by Onnela et al. [38] and Holme et al. [28] behave in a similar manner.

Claim 4.4. Barrat Clustering has a Constant Error Bound $\epsilon_{\max }$ equal to $\frac{3}{2\left|V^{*}\right|}$ and the probability of reaching this error is less than or equal to $1-\left(\begin{array}{c}\left|V^{*}\right|-3 \\ |V|-3\end{array}\right) /\left(\begin{array}{c}\left|V^{*}\right| \\ |V|\end{array}\right)$.

Deltacon. As mentioned before, Deltacon is a measure of the change in affinity, or random walk distance, between the nodes of a graph across a time step. Deltacon is defined as

$$
\operatorname{Deltacon}\left(G_{1}, G_{2}\right)=\operatorname{Dist}\left(S_{1}, S_{2}\right)=\sqrt{\sum_{i, j \in V_{1} \cup V_{2}}\left(\sqrt{s_{i j, 1}}-\sqrt{s_{i j, 2}}\right)^{2}},
$$


where $S_{1}, S_{2}$ are Affinity Matrices approximating the random-walk distance between nodes, calculated using matrix inversion

$$
S_{x}=\left[I+\epsilon^{2} D_{x}-\epsilon A_{x}\right]^{-1},
$$

with $A_{x}$ being the unweighted adjacency matrix of $G_{x}$ and $D_{x}$ being a diagonal matrix where $D_{x}[i, i]$ is the unweighted degree of node $i$. When $V_{1} \neq V_{2}$, it uses the union of the two node sets, and represents nodes not in $G_{1}$ or $G_{2}$ as disconnected, zero-degree nodes.

Claim 4.5. Deltacon is a Size Consistent statistic.

See the appendix for proof.

Claim 4.6. Deltacon is a Pseudo-Diverging statistic with an error bound $\epsilon_{\max }$ of $\sqrt{c}\left(\left|V^{*}\right|-\mid V_{1} \cup\right.$ $V_{2} \mid$ ) for some constant $c$.

Netsimile. The Netsimile statistic is a distance metric between pairs of graphs based on differences in a set of aggregate statistics. The Netsimile distance $N S\left(G_{1}, G_{2}\right)$ is defined as:

$$
N S\left(G_{1}, G_{2}\right)=\sum_{i=1}^{d} \frac{a b s\left(s_{G_{1}}^{i}-s_{G_{2}}^{i}\right)}{s_{G_{1}}^{i}+s_{G_{2}}^{i}},
$$

where $s^{1} \ldots s^{d}$ are graph aggregate features obtained by applying a set of aggregation methods to the local graph statistics produced by the nodes of a graph.

For example, if $F_{1}(n)$ and $F_{2}(n)$ are both features on node $n$ (like degree or local clustering coefficient) and $A g g_{1}(F), A_{g g_{2}}(F)$ are aggregation methods over all graph nodes (like mean or median of all nodes), the vector $\vec{s}_{G_{k}}$ consists of $\left\{\operatorname{Agg}_{1}\left(F_{1}(n)\right), \operatorname{Agg}_{2}\left(F_{1}(n)\right), \operatorname{Agg}_{1}\left(F_{2}(n)\right), \operatorname{Agg}_{2}\left(F_{2}(n)\right)\right\}$. The aggregates used are as follows: mean, median, standard deviation, skew, and kurtosis. The graph features used include: weighted node degree, local clustering coefficient, size of the egonet of a node, and outgoing edges of the egonet.

There are two possible sets of statistics that can be used: weighted ones that use weighted degree, weighted clustering coefficient, and so on, and unweighted ones that treat the graph as having unweighted edges only. We refer to these two approaches to Netsimile as Weighted and Unweighted Netsimile.

Claim 4.7. Weighted Netsimile is a Size Inconsistent statistic.

Claim 4.8. Unweighted Netsimile is a Size Consistent statistic.

Both proofs can be found in the appendix.

Claim 4.9. Unweighted Netsimile has a Constant Error Bound of 1.

Proof. As the Canberra distance always produces a value between 0 and 1, the Unweighted Netsimile score is similarly bounded, giving it a Constant Error Bound of 1 .

\subsection{Proposed Size Consistent Statistics}

We define a set of Size Consistent statistics designed to measure network properties similar to the previously described conventional statistics, but without the sensitivity to total network edge/node count. They are also designed such that the empirical estimations converge to the true values as quickly as possible.

These statistics use a matrix $\widehat{P}_{t}$, where $\widehat{p}_{i j, t}=\frac{w_{i j, t}}{\left|W_{t}\right|}$ which is an empirical estimate of $P_{t}$, obtained by normalizing the matrix $W$ of observed weights as shown in Figure 5. Obtaining this matrix can be thought of as a reversal of the sampling process shown in Figure 3. Although $\widehat{P}_{t}$ is only an 


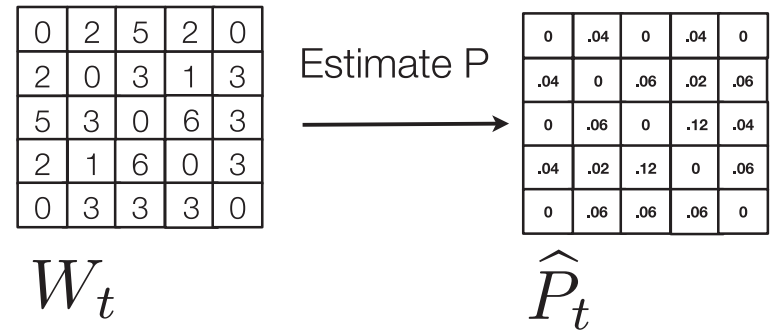

Fig. 5. Estimation of the $\widehat{P_{t}}$ matrix from the observed $W$ weights.

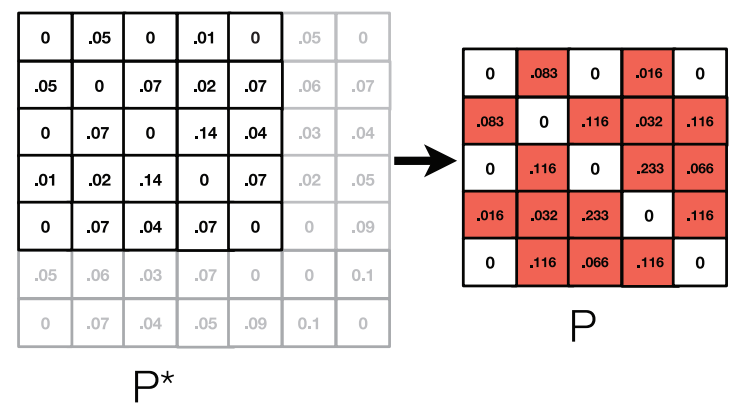

Fig. 6. Mass of the cells in $P$ increase as $|V|$ decreases.

estimate of $P_{t}$, it is an unbiased one, and given an increasingly large $\left|W_{t}\right|, \widehat{P}_{t}$ will equal $P_{t}$ in the limit. Therefore, empirical statistics that use $\widehat{P}_{t}$ in place of $P_{t}$ as their input will converge to the true statistic calculated on $P_{t}$ and the statistic converges w.r.t. $\left|W_{t}\right|$.

However, this does not guarantee that $\widehat{P}_{t}$ will converge in probability to $P_{t}^{*}$ as the number of nodes in $V_{t}$ increases. In fact, the value of any cell $\widehat{p}_{i j, t}$ is inversely proportional to $\left|V_{t}\right|$ : as both $\widehat{P}_{t}$ and $P_{t}^{*}$ are probability distributions which sum to 1 , the more cells in either matrix the lower the probability mass in each cell on average. This concentrating effect as $V_{t}$ is sampled from $V_{t}^{*}$ is demonstrated in Figure 6.

The solution to avoiding this concentration of probability mass is to introduce normalizing terms that negates the effect. These terms are $\overline{p^{*}}{ }_{t}=\frac{1}{\left|A_{t}^{*}\right|} \sum_{i j \in V_{t}^{*}} p_{i j, t}^{*}$ for $S_{k}\left(P_{t}^{*}\right)$ and the empirical term $\bar{p}_{t}=\frac{1}{\left|A_{t}\right|} \sum_{i j \in V} p_{i j, t}$ for $S_{k}\left(P_{t}\right)$, where $\left|A_{t}^{*}\right|$ and $\left|A_{t}\right|$ are the number of non-zero cells in $P_{t}^{*}$ and $P_{t}$, respectively. Replacing each $p_{i j, t}^{*}$ and $p_{i j, t}$ term in a statistic with $\frac{p_{i j, t}^{*}}{\overline{p_{t}^{*}}}$ and $\frac{p_{i j, t}}{\overline{p_{t}}}$ ensures that the statistic also converges as $\left|V_{t}\right|$ increases.

The utility of the $\overline{p^{*}}{ }_{t}$ and $\bar{p}_{t}$ terms is to normalize the probability mass concentration effect when the size of $|V|$ changes. As $P$ is a proper probability distribution and sums to a total of 1 , decreasing $|V|$ also causes the cells in $P$ to decrease and the probability mass in each cell to rise (illustrated in Figure 6). Normalizing by the mean of each non-zero cell $\bar{p}$ allows the terms of the consistent statistics to converge as $|V|$ increases and ensures that the bias remains small. Another way to consider this term is that $\bar{p}$ is a renormalization of $\overline{p^{*}}$, where $\overline{p^{*}}$ is the mean of the subset of $P^{*}$ cells that belong to $V$. As $\overline{p^{*}}$ is the sample mean approximating $\overline{p^{*}}$ it is an unbiased estimator of $\overline{p^{*}}$ and the inverse $\frac{1}{\overline{p^{*}} V}$ is a consistent estimator of $\frac{1}{\overline{p^{*}}}$ due to Slutsky's theorem. The regions spanned by each of these terms are shown in Figure 7. 


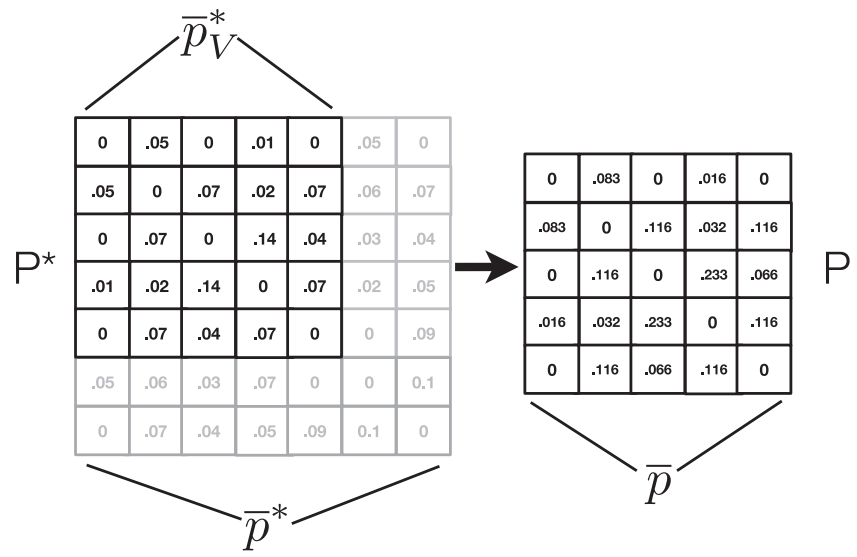

Fig. 7. Visualization of the regions averaged to obtain $\overline{p^{*}}, \bar{p}$, and $\overline{p^{*}}{ }_{V}$.

Probability Mass Shift. We introduce a new consistent statistic called Probability Mass Shift, which is a measure of change in the underlying $P^{*}$ matrices that produced two graphs. Similar to GED, when used on a dynamic network, it is a measure of the rate of change the network is experiencing. Unlike GED, it is consistent with respect to the size of the input graphs.

Let $P_{1}^{*}$ and $P_{2}^{*}$ be probability distributions over a node set $V^{*}$. Define the Probability Mass Shift between $P_{1}^{*}$ and $P_{2}^{*}$ to be

$$
M S\left(P_{1}^{*}, P_{2}^{*}\right)=\frac{1}{Z_{V^{*}}} \sum_{i j \in V^{*}}\left(\frac{p^{*}{ }_{i j, 1}}{\overline{p_{1}^{*}}}-\frac{p^{*}{ }_{i j, 2}}{\overline{p_{2}^{*}}}\right)^{2},
$$

where the term $\overline{p_{x}^{*}}=\frac{1}{\left|A^{*}\right|} \sum_{i j \in V^{*}} p_{i j}^{*}$ refers to the average value of non-zero cells in $P_{x}^{*}$ and $Z_{V^{*}}=$ $\left(\begin{array}{c}\left|V^{*}\right| \\ 2\end{array}\right)$.

Let the Probability Mass Shift between node subsets $V_{1}, V_{2} \subset V^{*}$ be

$$
M S\left(P_{1}, P_{2}\right)=\frac{1}{Z_{V_{\cap}}} \sum_{i j \in V_{\cap}}\left(\frac{p_{i j, 1}}{\overline{p_{1}}}-\frac{p_{i j, 2}}{\overline{p_{2}}}\right)^{2}
$$

where $V_{\cap}$ is the intersection of $V_{1}$ and $V_{2}, p_{i j, x}=\frac{p_{i j, x}^{*}}{\sum_{i j \in V_{n}} p_{i j, x}^{*}}$, and $\overline{p_{x}}=\frac{1}{|A|} \sum_{i j \in V_{\cap}} p_{i j}$.

Now define the empirical Probability Mass Shift $\widehat{M S}\left(G_{1}, G_{2}\right)$ to be

$$
\widehat{M S}\left(G_{1}, G_{2}\right)=\frac{1}{Z_{V_{n}}} \sum_{i j \in V_{n}}\left(\frac{\widehat{p}_{i j, 1}}{\overline{\widehat{p}_{1}}}-\frac{\widehat{p}_{i j, 2}}{\overline{\widehat{p}_{2}}}\right)^{2}
$$

where $\overline{\widehat{p}_{x}}=\frac{1}{\left|\widehat{A}_{x}\right|} \sum_{i j \in V_{n}} \widehat{p}_{i j},\left|\widehat{A_{x}}\right|=\sum_{i j \in V_{\cap}} I\left[w_{i j, x}>0\right]$, and $\widehat{p}_{i j, x}=\frac{w_{i j, x}}{\sum_{i j \in V_{n}} w_{i j, x}}$.

CLAIm 4.10. $\widehat{M S}\left(G_{1}, G_{2}\right)$ is a Size Consistent statistic that converges to $\operatorname{MS}\left(P_{1}^{*}, P_{2}^{*}\right)$.

See the appendix for proof.

We can improve upon the empirical version of the statistic by calculating the amount of bias for the $\left|W_{1}\right|,\left|W_{2}\right|$ values and subtracting off an estimate of this bias.

$$
\widehat{M S}\left(G_{1}, G_{2}\right)=\left(\left|\widehat{A_{1}}\right| \widehat{p}_{i j, 1}-\left|\widehat{A}_{2}\right| \widehat{p}_{i j, 2}\right)^{2}-\frac{1}{\left|W_{1}\right|}\left|\widehat{A}_{1}\right|^{2} \widehat{p}_{i j, 1}\left(1-\widehat{p}_{i j, 1}\right)-\frac{1}{\left|W_{2}\right|}\left|\widehat{A}_{2}\right|^{2} \widehat{p}_{i j, 2}\left(1-\widehat{p}_{i j, 2}\right)
$$


As $\left(\left|W_{1}\right|,\left|W_{2}\right|\right) \rightarrow(\infty, \infty)$ this bias correction term goes to 0 , so this does not affect the statistic's status as Size Consistent-it merely improves the rate of convergence toward the limit of the statistic.

ClAIM 4.11. The Empirical Mass Shift $\widehat{M S}\left(G_{1}, G_{2}\right)$ has a Constant Error Bound of

$$
\epsilon_{\max }=\frac{1}{8\left|V^{*}\right|^{2}}\left(\frac{\left|V^{*}\right|\left(2 W-\left|V^{*}\right|+2\right)}{W}\right)^{2} .
$$

The error will be less than this bound with probability $4\left(\begin{array}{c}\left|V^{*}\right|-2 \\ \left|V^{*}\right|-V\end{array}\right) /\left(\begin{array}{c}\left|V^{*}\right| \\ \left|V^{*}\right|-V\end{array}\right)$.

Probabilistic Degree Distance. The empirical Probabilistic Degree Distance is a delta statistic that measures the difference between the degree distributions of two graphs in a Size Consistent manner. It is defined as

$$
\widehat{P D D}\left(G_{1}, G_{2}\right)=\sum_{\text {bin }} \in \text { Bins }\left(\frac{1}{\left|V_{1}\right|} \sum_{i \in V_{1}} I\left[\widehat{P D}_{i}\left(G_{1}\right) \in \operatorname{bin}_{k}\right]-\frac{1}{\left|V_{2}\right|} \sum_{i \in V_{2}} I\left[\widehat{P D}_{i}\left(G_{2}\right) \in \operatorname{bin}_{k}\right]\right)^{2},
$$

where $\widehat{P D}_{i}\left(G_{x}\right)=\frac{1}{\overline{\bar{p}}_{\text {row }, x}} \sum_{j \in V_{x}} \widehat{p}_{i j, x}$ is the empirical probability degree of a node, Bins is a set of bins dividing the range of all probability degrees across $G_{1}$ and $G_{2}$ into $k$ equal-sized segments, and $\widehat{\bar{p}}_{\text {row }, x}=\frac{1}{\left|V_{x}\right|}$. The empirical probability degree can be rewritten as $\widehat{P D}_{i}\left(G_{x}\right)=\left|V_{x}\right| \sum_{j \in V_{x}} \widehat{p}_{i j, x}$.

The true Probabilistic Degree Difference $\operatorname{PDD}\left(P_{1}^{*}, P_{2}^{*}\right)$ is

$$
P D D\left(P_{1}^{*}, P_{2}^{*}\right)=\sum_{\text {bin }_{k} \in \text { Bins }}\left(\frac{1}{\left|V^{*}\right|} \sum_{i \in V^{*}} I\left[P D_{i}\left(P_{1}^{*}\right) \in \operatorname{bin}_{k}\right]-\frac{1}{\left|V^{*}\right|} \sum_{i \in V^{*}} I\left[P D_{i}\left(P_{2}^{*}\right) \in \operatorname{bin}_{k}\right]\right)^{2},
$$

where $P D_{i}\left(P_{x}^{*}\right)=\frac{1}{\bar{p}^{*} \text { row } x} \sum_{j \in V^{*}} p^{*}{ }_{i j, x}$ and $\overline{\bar{p}}_{\text {row }, x}=\frac{1}{\left|V^{*}\right|}$.

Claim 4.12. The empirical Probability Degree Distance is a Size Consistent statistic.

See the appendix for proof.

Like the Mass Shift statistic, a bias correction term of $-\frac{\left(p_{k, 1}\right)\left(1-p_{k, 1}\right)}{\left|V_{1}\right|}-\frac{\left(p_{k, 2}\right)\left(1-p_{k, 2}\right)}{\left|V_{2}\right|}$ can be applied to the PDD, where $p_{k, x}$ is the probability of a node selected at random from $V_{x}$ belonging to bin $k$, giving an empirical statistic of

$$
\begin{aligned}
\widehat{P D D}\left(G_{1}, G_{2}\right)= & \sum_{\text {bin }_{k} \in \text { Bins }}\left(\frac{1}{\left|V_{1}\right|} \sum_{i \in V_{1}} I\left[P D_{i}\left(P_{1}\right) \in \operatorname{bin}_{k}\right]-\frac{1}{\left|V_{2}\right|} \sum_{i \in V_{2}} I\left[P D_{i}\left(P_{2}\right) \in b i n_{k}\right]\right)^{2}-\frac{\left(p_{k, 1}\right)\left(1-p_{k, 1}\right)}{\left|V_{1}\right|} \\
& -\frac{\left(p_{k, 2}\right)\left(1-p_{k, 2}\right)}{\left|V_{2}\right|} .
\end{aligned}
$$

ClaIm 4.13. The Empirical Probability Degree $\widehat{P D D}\left(G_{1}, G_{2}\right)$ has a Constant Error Bound of

$$
\epsilon_{\max }=\frac{\left.2\left(\left|V^{*}\right|-1\right)^{2}\right)+2}{\left|V^{*}\right|^{2}}
$$

and the probability of attaining this error is at most $\left(\begin{array}{c}\left|V^{*}\right|-1 \\ \left|V^{*}\right|-|V|-1\end{array}\right) /\left(\begin{array}{c}\left|V^{*}\right| \\ \left|V^{*}\right|-|V|\end{array}\right)$.

The proof can be found in the appendix.

Triangle Probability. As the name suggests, the TP statistic is an approach to capturing the transitivity of the network and an alternative to traditional clustering coefficient measures. Define the 
$\mathrm{TP}$ as

$$
T P\left(P^{*}\right)=\frac{1}{Z^{*}} \sum_{i j k \in V^{*}}\left(\frac{1}{\left(\overline{p^{*}}\right)}\right)^{3} p_{i j}^{*} p_{i k}^{*} p_{j k}^{*}=\frac{1}{Z^{*}} \sum_{i j k \in V^{*}}\left|A^{*}\right|^{3} p_{i j}^{*} p_{i k}^{*} p_{j k}^{*} .
$$

The empirical version on $G$ is

$$
\widehat{T P}(G)=\frac{1}{Z} \sum_{i j k \in V} \widehat{|A|}^{3} \widehat{p}_{i j} \widehat{p}_{i k} \widehat{p}_{j k}
$$

where $Z^{*}=\left(\begin{array}{c}\left|V^{*}\right| \\ 3\end{array}\right)$ and $Z=\left(\begin{array}{c}|V| \\ 3\end{array}\right)$.

Claim 4.14. TP is a Size Consistent statistic.

See the appendix for proof.

Just as with Mass Shift, we can obtain a bias correction term, making the empirical TP:

$$
\widehat{T P}(G)=\frac{1}{Z} \sum_{i j k \in V} \widehat{|A|}^{3}\left(\widehat{p}_{i j} \widehat{p}_{i k} \widehat{p}_{j k}-\frac{3}{|W|} \widehat{p}_{i j} \widehat{p}_{i k} \widehat{p}_{j k}\right) \text {. }
$$

ClaIm 4.15. The Empirical Triangle Probability $\widehat{T P}(G)$ has a Constant Error Bound of

$$
\epsilon_{\max }=\left(\frac{\left(2\left|V^{*}\right|+3\right)\left(2 W-\left|V^{*}\right|+3\right)}{12 W\left|V^{*}\right|}\right)^{3},
$$

and the probability that it achieves this bound is less than or equal to $1-\left(\begin{array}{c}\left|V^{*}\right|-3 \\ |V|-3\end{array}\right) /\left(\begin{array}{c}\left|V^{*}\right| \\ |V|\end{array}\right)$.

The proof can be found in the appendix.

\section{ANOMALY DETECTION PROCESS}

In order to perform anomaly detection on a dynamic network, the collection of messages needs to first be converted into a sequence of graph instances. Each message consists of a pair of nodes and an associated timestamp. After picking a time step width $\Delta$, the graph at each time step $t$ is created by adding all messages falling between $t$ and $t+\Delta$ to matrix $W_{t}$, producing a sequence of graphs. The algorithm is described in Figure 8.

Then, a statistic value needs to be calculated for every graph instance in the stream. As the length of the stream is usually short compared to the size of the graphs, the computational complexity depends on the cost of calculating the network statistics on the largest graph instance. In order to calculate our consistent statistics, $\widehat{P}_{t}$ is estimated by normalizing the observed messages $W$. Then, the network statistic scores are calculated at each time step. This generates a set of standard time series, which can be analyzed with traditional time series anomaly detection techniques.

Selection of a proper $\Delta$ time step width is crucial. Due to the nature of Size Consistent statistics, larger values of $\Delta$ will reduce the error associated with statistical bias, but larger values also reduce the granularity of the detection algorithm making it harder to pinpoint the exact time that the anomaly occurred.

Now that we have a stream of graphs we can perform the anomaly detection process. For every time step $t$, the graph at $G_{t}$ becomes the test graph and the graphs $G_{t-1}, G_{t-2} \ldots G_{t-k}$ become the null distribution examples (here, we use $k=50$ ). By applying $\widehat{S}_{k}$ to each graph we obtain both the test point and the null distribution. Given a certain $p$-value $\alpha$, we then set the critical points to be the values that reject the most extreme $\alpha / 2$ values from the null distribution on both sides. If the test point $\widehat{S}_{k}\left(G_{t}\right)$ falls outside of these critical points we can reject the null hypothesis and raise an anomaly flag. This detection algorithm is described in Figure 9. 
GraphProcess (messages, $\Delta)$ :

requires messages, $\Delta$

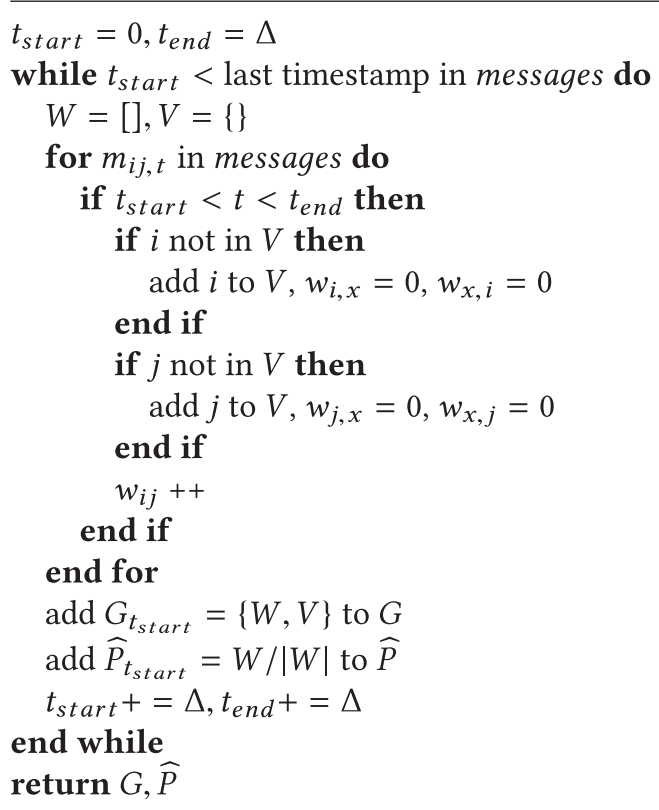

Fig. 8. Creation of the dynamic graph sequence from message stream using time step width $\Delta$.

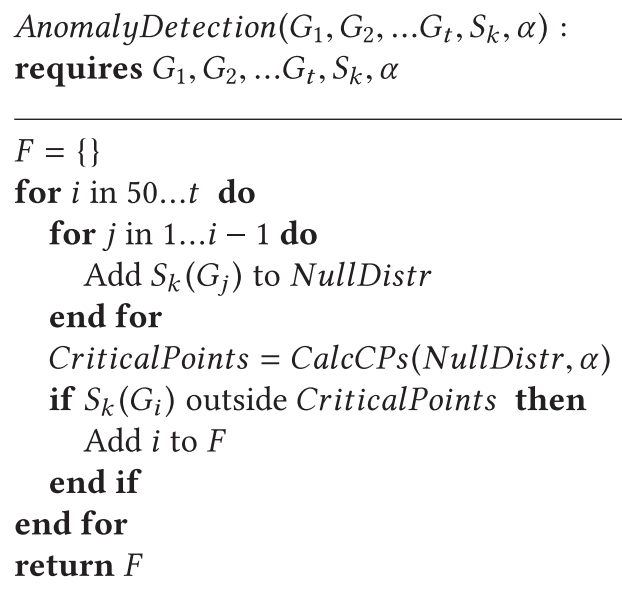

Fig. 9. Anomaly detection procedure for a graph stream $\left\{G_{1} \ldots G_{t}\right\}$, graph statistic $S_{k}$ and $p$-value $\alpha$.

\subsection{Complexity Analysis}

Statistics like probability mass and probabilistic degree can be calculated at each step in $O\left(\left|A_{t}\right|\right)$ time, making their overall complexity $O\left(\left|A_{t}\right| t\right)$, where $\left|A_{t}\right|$ is the number of non-zero elements in $W_{t}$. TP, on the other hand, is more expensive as some triangle counting algorithm must be applied. The fastest counting algorithms typically run in $O\left(|V|^{k}\right)$ time where $2<k<3$, making the overall 
complexity $O\left(|V|^{k} t\right)$ for the whole stream. However, if we make the assumption that the maximum number of neighbors of any node is bounded by $n_{\max }$, we can approximate the triangle count with $O\left(|V| n_{\max }^{2} t\right)$. Note that any other statistic-based approach such as Netsimile that utilizes triangle count or clustering coefficient must make the same approximations in order to run in linear time.

\section{EXPERIMENTS}

Now that we have established the properties of Size Consistent and Inconsistent statistics, we demonstrate the tangible effects of these statistical properties using synthetic, semi-synthetic, and real-world datasets. The objective for the synthetic and semi-synthetic experiments is to maximize the true positive detection rate (where a true positive means flagging a graph generated with anomalous parameters) and minimize the false positive rate (where a false positive means flagging a graph with unusual edge count or node count but generated with normal parameters). The realworld experiments are an exploratory analysis, demonstrating how to discover and explain events in a real-world dynamic graph.

We compare each of the consistent statistics to the conventional one they were intended to replace: GED to probability mass shift, degree distribution difference to probabilistic degree difference, and Barrat weighted clustering to TP. In addition, we also compare the performance of the consistent statistics to Netsimile and Deltacon. Netsimile is an aggregate statistic that attempts to measure graph differences in a variety of dimensions and as such can be applied to find many types of anomalies. Deltacon on the other hand measures graph differences through the distances between nodes in the graphs and attempts to find anomalies of an entirely different type than the consistent statistics.

\subsection{Synthetic Data Experiments}

In order to create data with specific known properties, we used generative graph models. There are following four types of graphs generated:

(1) Normal graph examples that are used to create the null distribution for a hypothesis test.

(2) Edge false positive graphs that are generated using the same model parameters but with additional sampled edges.

(3) Node false positive graphs that are generated using the same model parameters but with additional sampled nodes.

(4) True positive graphs that are generated with a normal number of edges and nodes but different model parameters.

The first three sets of graphs are created with the same generative model but with varying edges and nodes in the output graphs, while the last set uses a different generative model. An illustration of the null distribution, false positive distribution with additional edges, and true positive distribution is shown in Figure 10. Figure 11 describes the synthetic data generation algorithm in more detail.

First a set of normal graph examples are created using the process described in (1) which form the null distribution graphs. A statistic is calculated for each graph example and given an $\alpha$ value the two critical points are found. Then, a false positive graph set is created using either (2) or (3) and a true positive graph set created using (4) and statistics calculated for each. The percentage of false positive graphs outside the critical points becomes the false positive rate, while the percentage of true positive graphs becomes the true positive rate. By varying the value of $\alpha$ and plotting the true positive versus false positive rate for each value, we can create an ROC curve showing the tradeoff of true anomalous instances found versus falsely flagged instances. 


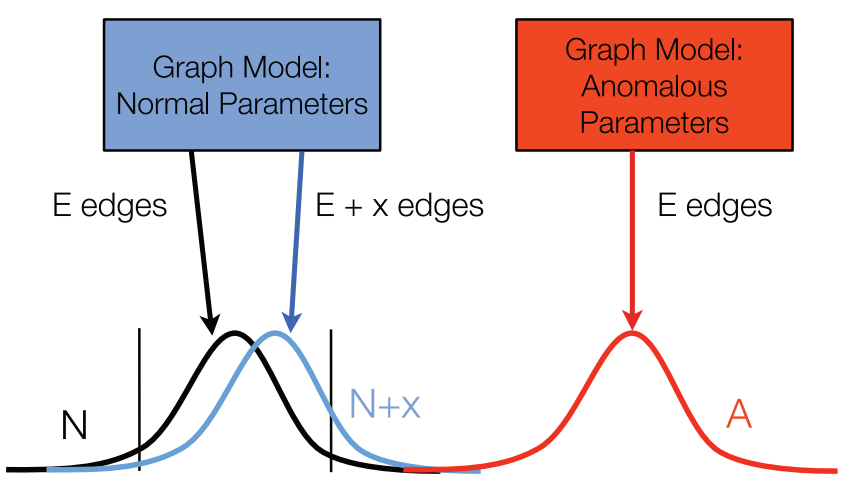

Critical Points

Fig. 10. Diagram of synthetic experiments and the three sets of generated graphs.

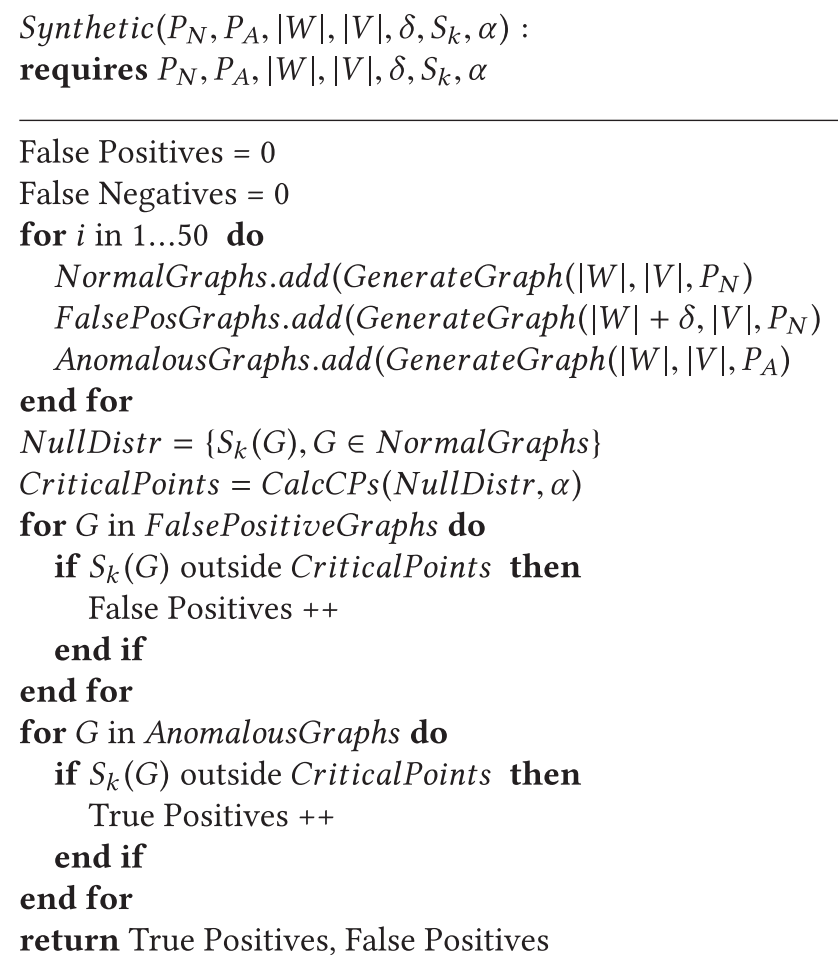

Fig. 11. Synthetic data experimental procedure for statistic $S_{k}$ using normal probability matrix $P_{N}$, anomalous probability matrix $P_{A}$, and $\Delta$ additional edges in False Positive graphs.

The circle on the ROC curves represents selecting a $p$-value of 0.05 . The number of edges in the normal and true positive graphs ranges from $300 \mathrm{k}$ to $400 \mathrm{k}$, while the edge false positive graphs have $400 \mathrm{k}-500 \mathrm{k}$, and the number of nodes in the normal and true positive graphs is $25 \mathrm{k}$, while the node false positive graphs have $30 \mathrm{k}$. An equal number of graphs of each type were generated. For a statistic that detects the model changes reasonably well, we expect the false positive distribution to be very close to the null distribution, while the anomalous distribution is significantly different. 


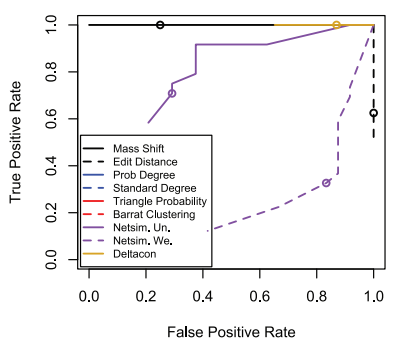

(a) Mass Shift

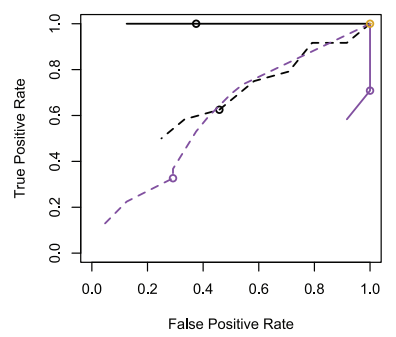

(d) Mass Shift

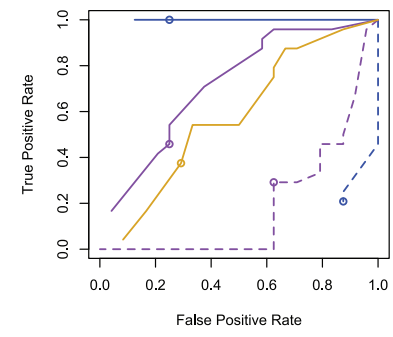

(b) Probability Degree

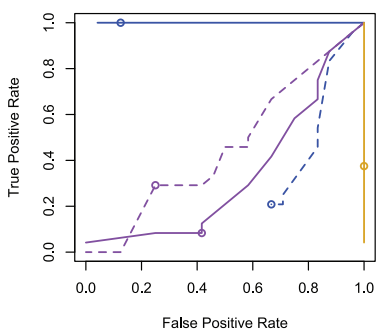

(e) Probability Degree

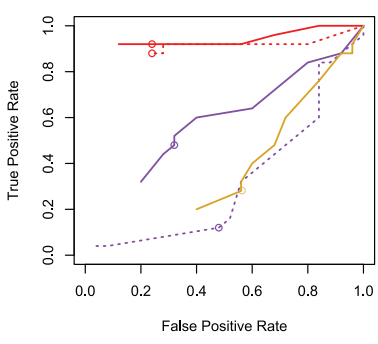

(c) Triangle Probability

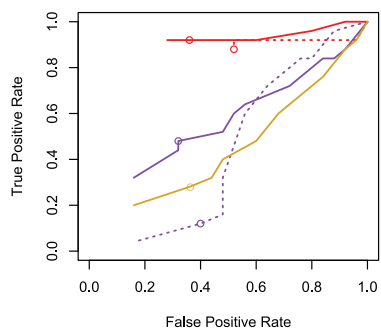

(f) Triangle Probability

Fig. 12. $(a-c)$ : ROC curves with false positives caused by extra edges; $(d-f)$ : ROC curves with false positives due to extra nodes. Points represent $\alpha=0.05$. Dashed lines indicate Size Inconsistent counterparts to Size Consistent statistics. Black is Probability Mass Shift and Edit Distance; Blue is Probability Degree and Standard Degree; Red is Triangle Probability and Barrat Clustering; Purple is Unweighted and Weighted Netsimile; and Yellow is Deltacon. In most cases, the Size Consistent statistic has superior performance to the Size Inconsistent counterpart, and this is always true for the Size Consistent statistics we introduced.

Ideal performance on the ROC curve would be a horizontal line across the entire top of the plot: this would indicate perfect performance in detecting true positive graphs even at low $p$-value, and a false positive rate that is low until the $p$-value is increased. For comparison a diagonal line with a slope of 1 would indicate random performance where each false positive and true positive graph is flagged as anomalous using an unbiased coin flip. Any statistic that has a curve below this line has more sensitivity to the additional edges or nodes of the false positive graphs than to the model changes of the true positive graphs. Some of the statistics evaluated even have a vertical line at the right of the plot: this indicates that no matter the $p$-value picked all false positive graphs are being flagged but not all true positive graphs are flagged; this is the worst possible space for the statistic to be in.

To evaluate delta statistics, graphs were generated in pairs, the first being from the normal/false positive/true positive model, while the other always from the normal model, and the delta statistic calculated between them.

To test the performance of graph change statistics like GED and probability mass shift, synthetic data was generated using a mixture model that either samples edges from a static normal graph instance from (1) or from an anomalous graph from (4). The initial graph has a power-law degree distribution with an exponent of 2.0 and was generated using a Chung-Lu sampling process, while the alternative graph was generated with an Erdos-Renyii graph model. The normal model draws edge samples only from the initial graph, while the alternative model draws $5 \%$ of the edges from the alternative graph. The performance of these statistics is shown in Figure 12(a) and (d). Mass Shift strictly dominates the other statistics as either the edges or nodes changes. 
To determine ability to detect degree distribution changes synthetic graphs were also generated using a Chung-Lu process; however, anomalous graph instances were generated by altering the exponent parameter of the power law determining degree distribution rather than using a mixture model. The normal graph instances have a power-law degree distribution with an exponent of 2.0, while the true positive graph has an exponent of 1.8. The performance is shown in Figure 12(b) and (e).

The transitivity experiments were done by creating graphs with a varying amount of triangle closures. To create each graph, a KPGM model with a seed of $\left[\begin{array}{cc}0.4 & 0.3 \\ 0.3 & 0\end{array}\right]$ is used to sample an initial edge set. These parameters were selected to create a graph with a branching pattern with few natural triangles. Then, with probability $\rho$ each edge is removed and replaced with a triangle closure by performing a random walk (identical to the technique used in the Transitive Chung Lu model [40]). The normal graphs were generated with a rho of 0.05 , while the alternative graphs had a rho of 0.055 . The results are in Figure 12(c) and (f).

Across all of the examined statistics, the Size Consistent statistics introduced in this article have superior performance attaining near-perfect ROC curves. In most cases, the Size Consistent statistics (solid) outperform their Size Inconsistent counterparts (dashed) by a large margin, and the statistics we introduced always outperform their counterparts.

As some of the statistics are below a line with a slope of 1 (i.e., random performance), one might ask whether the statistics would be improved by "flipping" them by inverting their output to create a mirror image. While that would improve the performance in these specific tests, it would also mean that any data drawn from the null distribution would become a false positive by the inverted statistic, making its use as the test statistic to a hypothesis test completely invalid.

Figures 13 and 14 demonstrate the convergence properties of each statistic as the number of edges or nodes, respectively, sampled from a semi-synthetic model is increased. The heat maps show the difference in edges/nodes between a pair of consecutive graphs on the $y$-axis and the size of the smaller of the two graphs on the $x$-axis; the line plots show the convergence along the lines $G_{1}=G_{2}$ versus $G_{1}=2 * G_{2}$. The semi-synthetic model in question samples from the network of all communications in the University e-mail dataset. Figure 13 includes graphs with between $500 \mathrm{k}$ and 10 million total messages between $12 \mathrm{k}$ nodes; Figure 14 has between $1 \mathrm{k}$ and $42 \mathrm{k}$ nodes using the true message count from the sampled data (equivalent to infinite edge samples).

Figure 13(a)-(c), (k), (j), and (p) shows the consistent statistics Mass Shift, Probabilistic Degree, Unweighted Netsimile, Deltacon, TP, and Barrat Clustering, respectively. As consistent statistics, as the minimum size of the graphs (the $x$-axis) increases the output of the statistics should converge to the same value regardless of the difference in size. Mass Shift and Unweighted Netsimile are in the process of converging as the line plots show: Mass Shift is converging slightly faster to its limit. Probabilistic Degree has already converged on graphs of this size and shows random noise around the limit only. Deltacon appears to be converging very slowly to a limit. The other statistics are either diverging or have no true limit.

Figure 14 shows the node convergence behavior. Mass Shift, Probability Degree, and TP have already mostly converged and show just random noise around their limits. The other statistics, including those that are technically Size Consistent, show no sign of converging. This is because of those statistics that do converge they only reach their limit when close to the entire node set of the underlying model is observed. This means that they effectively behave like size divergent statistics when the node count is not close to the finite limit, and their performance suffers for it.

\subsection{Semi-Synthetic Data Experiments}

Although synthetically driven experiments have the advantage of complete control over the network properties of the generated graphs, these experiments give inherently artificial results 


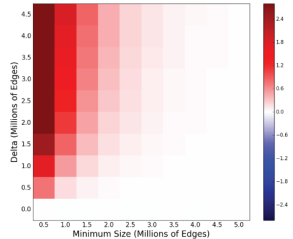

(a) Mass Shift

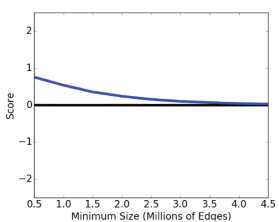

(e) Mass Shift

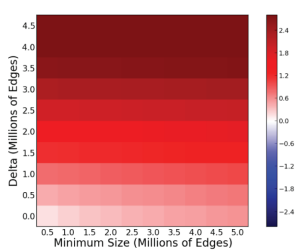

(i) Graph Edit Distance

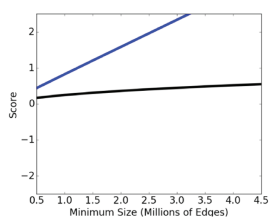

(m) Graph Edit Distance

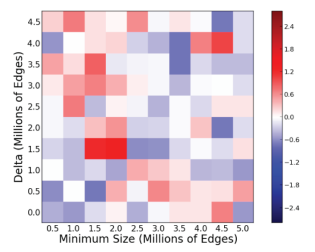

(b) Probability Degree

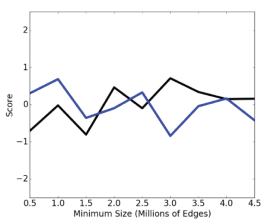

(f) Probability Degree

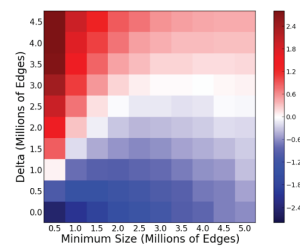

(j) Degree Distance

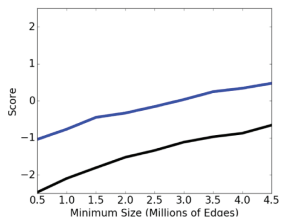

(n) Degree Distance

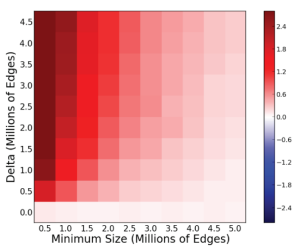

(c) Unweighted Netsimile

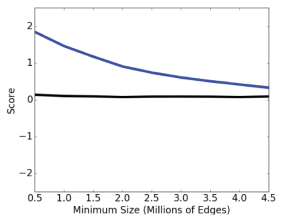

(g) Unweighted Netsimile

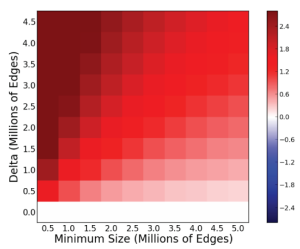

(k) Weighted Netsimile

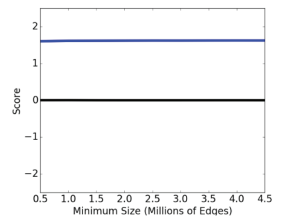

(o) Weighted Netsimile

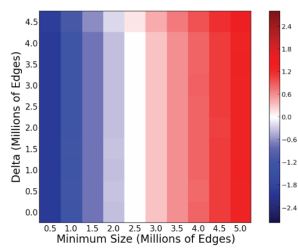

(d) Deltacon

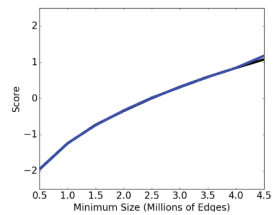

(h) Deltacon

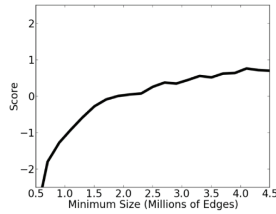

(I) Triangle Probability

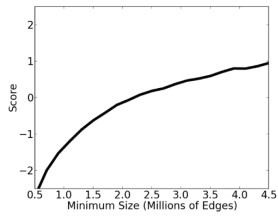

(p) Barrat Clustering

Fig. 13. Convergence behavior of each statistic when varying edge count. The heatmap $x$-axis is the minimum size of the graph pair, while the $y$-axis is the difference in size. The blue line on the line plots is the diagonal $x=y$ from the heatmap, while the black line is $y=0$. (a-d) and $(e-h)$ show the behavior of the Theoretically Consistent statistics Mass Shift, Probability Degree, Unweighted Netsimile, and Deltacon. (i$\mathrm{k})$ and $(\mathrm{m}-\mathrm{o})$ show the behavior of Theoretically Inconsistent statistics Edit Distance, Standard Degree, and Weighted Netsimile. (I) and (p) are Triangle Probability and Barrat Clustering. Theoretical Consistency requires that regardless of the relationship between the $x$ and $y$ dimensions, the statistics will converge to the same value; this can be seen in the line plots of Consistent statistics, while the Inconsistent statistics diverge.

and the utility of any conclusions drawn from those experiments depends heavily on the comprehensiveness of the experiments. To ensure that these results generalize to more realistic scenarios I have also evaluated them using a set of semi-synthetic experiments where the normal and false positive graph examples of (1)-(3) are sampled from real-world networks and the true positive anomaly examples of (4) are artificially inserted. These experiments show that the proposed consistent statistics are superior at discovering anomalies inserted into real-world data.

The first step in generating the graph sets is to aggregate all graph instances from a dynamic network source into a single graph example, which becomes our normal graph source. All normal 


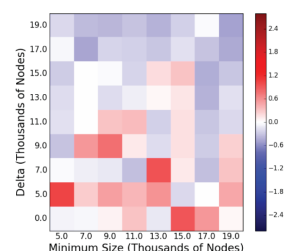

(a) Mass Shift

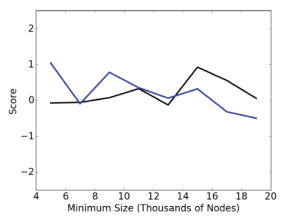

(e) Mass Shift

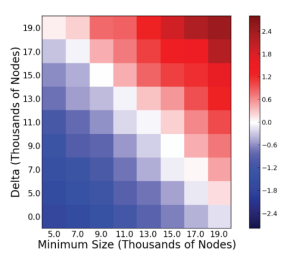

(i) Graph Edit Distance

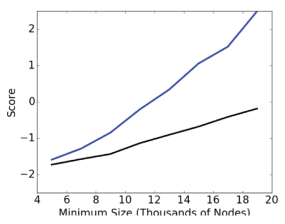

(m) Graph Edit Distance

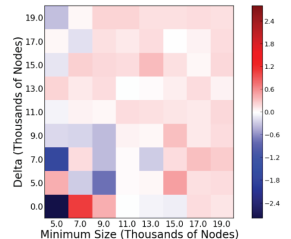

(b) Probability Degree

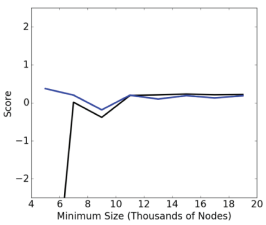

(f) Probability Degree

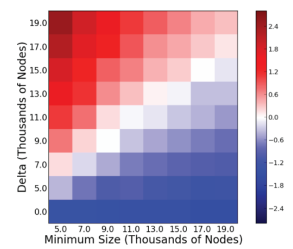

(j) Degree Distance

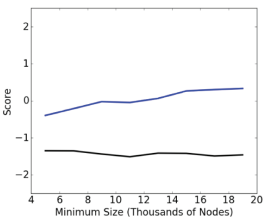

(n) Degree Distance

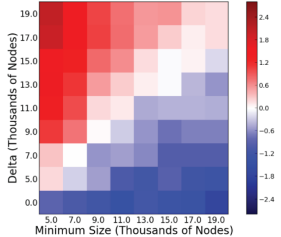

(c) Unweighted Netsimile

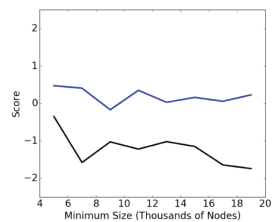

(g) Unweighted Netsimile

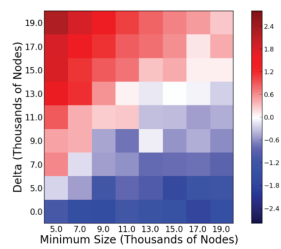

(k) Weighted Netsimile

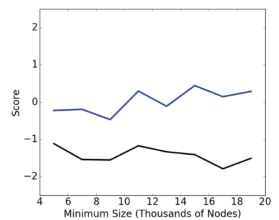

(o) Weighted Netsimile

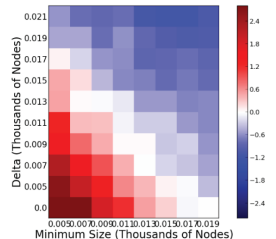

(d) Deltacon

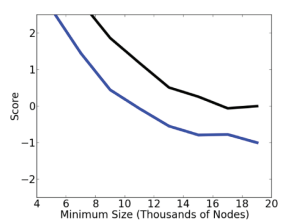

(h) Deltacon

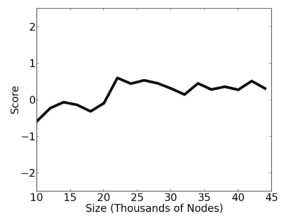

(I) Triangle Probability

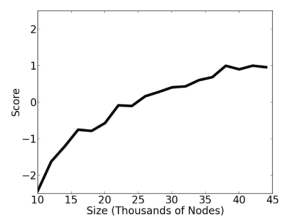

(p) Barrat Clustering

Fig. 14. Convergence behavior of each statistic when varying node count. The heatmap $x$-axis is the minimum size of the graph pair, while the $y$-axis is the difference in size. The blue line on the line plots is the diagonal $x=y$ from the heatmap, while the black line is $y=0$. (a-d) and $(e-h)$ show the behavior of the Theoretically Consistent statistics Mass Shift, Probability Degree, Unweighted Netsimile, and Deltacon. (i$\mathrm{k})$ and $(\mathrm{m}-\mathrm{o})$ show the behavior of Theoretically Inconsistent statistics Edit Distance, Standard Degree, and Weighted Netsimile. (I) and (p) are Triangle Probability and Barrat Clustering. Although Unweighted Netsimile and Deltacon are Theoretically Consistent, they do not converge on graphs of the size we used here making them Empirically Inconsistent; Mass Shift and Probability Degree do converge on graphs of this size and are Empirically Consistent.

graph examples are generated from this source graph by first sampling an active node set, obtaining the subgraph over those active nodes, then sampling edges with replacement to create the sample graph. By aggregating all instances over time, we smooth out any variations that occur over the lifespan of the network and obtain the "average" behavior of the network to use as our normal examples. False positive examples are created by sampling additional nodes or edges from the same source network. 


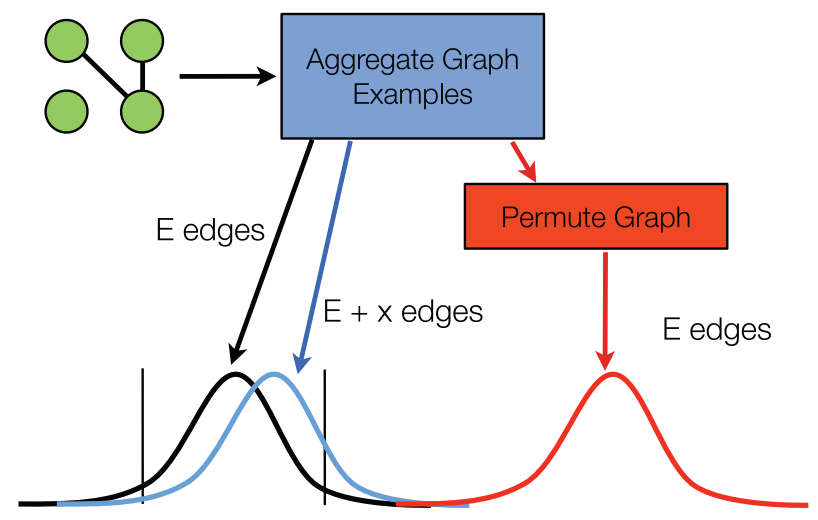

Critical Points

Fig. 15. Diagram of semi-synthetic experiments and the three sets of generated graphs.

SemiSynthetic $\left(P_{N}, P_{A},|W|,|V|, \delta, S_{k}, \alpha\right)$ :

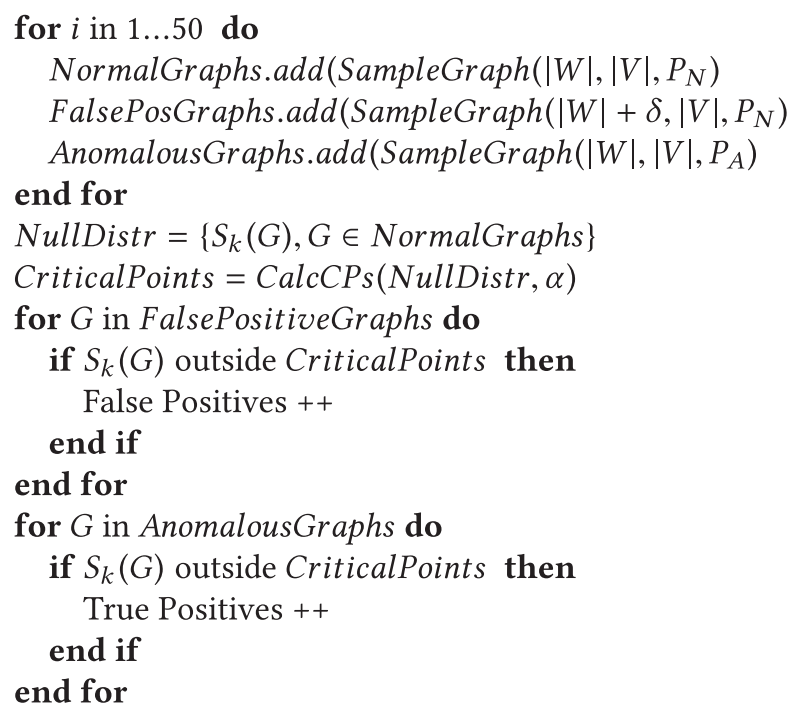

Fig. 16. Semi-synthetic data experimental procedure for statistic $S_{k}$ using normal probability matrix $P_{N}$, anomalous probability matrix $P_{A}$, and $\Delta$ additional edges in False Positive graphs.

True positive examples are sampled from a separate, alternate source instance that is created by permuting the original source graph in some way. To generate network change anomalies the alternate source has $5 \%$ of its edges selected uniformly at random compared to the source; degree distribution anomalies are generated by taking $30 \%$ of the edges of high degree nodes (high degree meaning in the top 50\% of nodes) and assigning them uniformly at random; and transitivity anomalies are generated by performing triangle closures by selecting an initial node, randomly walking two steps, then linking the endpoints of the walk to form a triangle. The semi-synthetic data generation process is shown in Figure 15 and the exact algorithm for generating the data is described in Figure 16. The input $P_{N}$ is created by dividing the aggregated normal graph described 


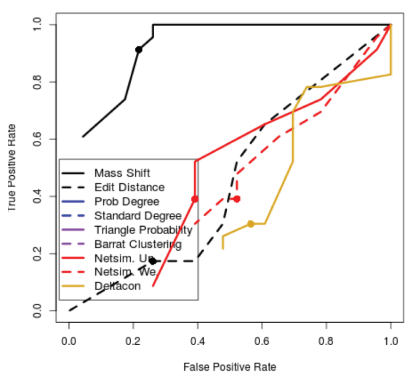

(a) Mass Shift

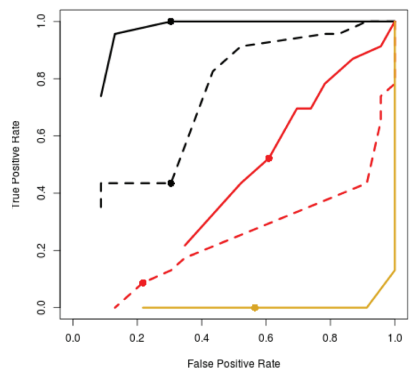

(d) Mass Shift

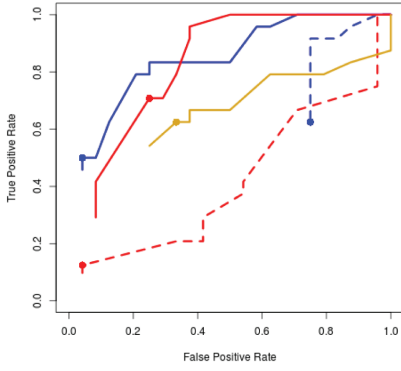

(b) Probability Degree

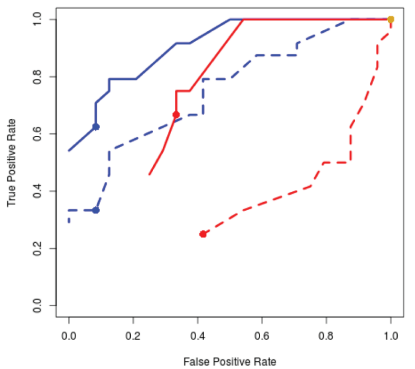

(e) Probability Degree

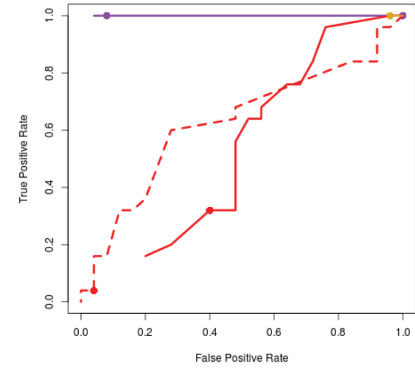

(c) Triangle Probability

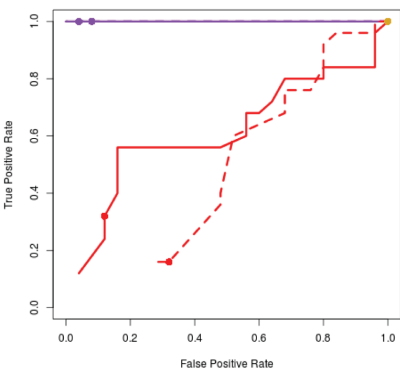

(f) Triangle Probability

Fig. 17. $(a-c)$ : ROC curves with false positives caused by extra edges; $(d-f)$ : ROC curves with false positives due to extra nodes. Points represent $\alpha=0.05$. Dashed lines indicate Size Inconsistent counterparts to Size Consistent statistics. Black is Probability Mass Shift and Edit Distance; Blue is Probability Degree and Standard Degree; Red is Triangle Probability and Barrat Clustering; Purple is Unweighted and Weighted Netsimile; and Yellow is Deltacon. In most cases, the Size Consistent statistic has superior performance to the Size Inconsistent counterpart, and this is always true for the Size Consistent statistics we introduced.

above by $|W|$ and the input $P_{A}$ is created by modifying the aggregated normal graph in one of the ways described above and then dividing by $|W|$.

The dataset used for the underlying graph was the University E-mail dataset described in Section 6.3 used in the real data experiments; when aggregated this data forms a graph with 54,102 total nodes and 5,236,591 total messages. Edge false positives are generated by creating graphs with $20 \mathrm{k}$ nodes and either $400 \mathrm{k}$ or $600 \mathrm{k}$ edges, while node false positives are generated by sampling either $20 \mathrm{k}$ or $30 \mathrm{k}$ nodes and sampling edges equal to 20 times the number of nodes. Sampling edges as a ratio of nodes in the node false positive experiment is to hold the density of the graphs constant.

We analyze the performance of the statistics using the same ROC approach as with the synthetic data. Figure 17 shows the resulting ROC curves. As with the synthetic experiments (a)-(c) show mass shift statistics, degree distribution statistics, and transitivity statistics, respectively, when the false positives are generated with additional edges, while (d)-(f) have false positives generated via additional nodes. The proposed consistent statistics have superior performance in most cases, and none of the competing statistics perform well in both the additional edges and additional nodes scenarios.

\subsection{Real Data Experiments}

Now let us investigate the types of anomalies found when these statistics are applied to three real-world networks and contrast these events to those found by other detectors. The first dataset 
Table 3. Precision and Recall of Statistics on Enron Anomalies

\begin{tabular}{c|c|c}
\hline & Precision & Recall \\
\hline Consistent statistics & 0.5 & 0.75 \\
Deltacon & 0.428 & 0.375 \\
Unweighted Netsimile & 0.125 & 0.125 \\
Weighted Netsimile & 0 & 0 \\
Edge count & 0.166 & 0.125 \\
\hline
\end{tabular}

is the Enron communication data, a subset of e-mail communications from prominent figures of the Enron corporation (150 individuals and 47,088 total messages) with a time step width of one week used in articles, such as Priebe et al. [41]. The second is the University E-mail data, e-mail communications of students from one university in the 2011-2012 school year (54,102 individuals, 5,236,591 total messages), sampled daily and described in detail in the paper by LaFond et al. [31]. The third is a Facebook network subset made up of postings to the walls of students in the 20072008 school year (444,829 individuals and 4,171,383 total messages), also from the same university and sampled daily. The Facebook dataset was also used in a paper by LaFond [20] and is described there in more detail.

Table 3 shows the precision and recall of each statistic on the Enron dataset using the highlighted events as the ground truth. Here, the Size Consistent statistics of Mass Shift, Probability Degree and TP are taken in an aggregate, as they are detectors for specific types of anomalies rather than general detectors like Netsimile or Deltacon. Graph instances that score higher on the Deltacon statistic than the threshold described in the original paper are flagged as anomalies. Both versions of Netsimile perform poorly due to the Canberra distance making them sensitive to graphs with a smaller edge count and node count; the Unweighted version performs slightly better, detecting one true positive, due to its Size Consistent nature.

Of the eight true anomalies that we used as the ground truth in the Enron dataset, the aggregate Consistent Statistics finds six. Three of these were detected by Mass Shift, one was Probability Degree, one was TP, and another was detected by both Mass Shift and Probability Degree. Deltacon found three of these anomalies, while Unweighted Netsimile and Edge Count found one each. Weighted Netsimile did not detect any of the anomalies.

Figure 18(a) shows the results of multiple statistics when applied to the set of e-mail data from the Enron corporation, including our three proposed statistics, the raw message count, Netsimile, and Deltacon. Time step 143 represents the most significant event in the stream, Jeffrey Skilling's testimony before congress on February 6, 2002. The detected triangle anomalies at time steps 5060 coincide with Enron's price manipulation strategy known as "Death Star" that was put into action in May 2000. Other events include The CEO transition from Lay to Skilling in December 2000, the "asshole" conference call featured prominently in the book "The Smartest Guys in the Room,” and Lay approaching Skilling about resigning.

Netsimile has difficulty detecting most of important events in the Enron timeline. Although it accurately flags the time of the Congressional hearings, the other points flagged, particularly early on, do not correspond to any notable events and are probably false positives due to the artificial sensitivity of the algorithm in very sparse network slices.

Deltacon detects a greater range of events than Netsimile but still fails to detect several important events such as the price manipulation and Skilling's attempted resignation. In general, it generates detections more frequently in the region between May and December 2001, which is also the region of highest message activity, and fails to generate detections in times with fewer messages. 


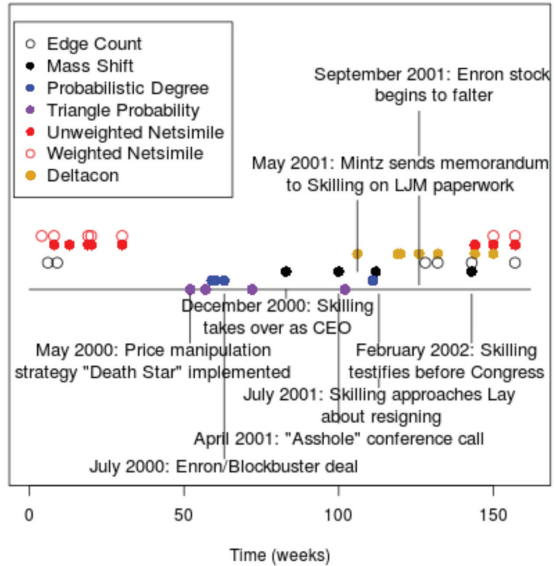

(a)

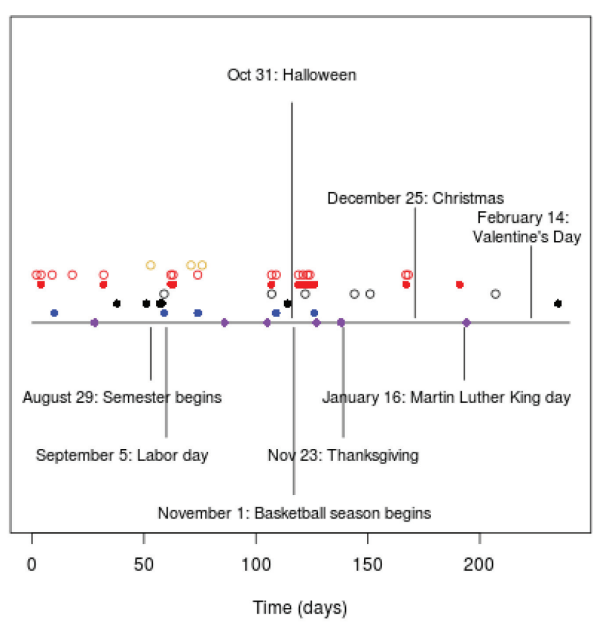

(b)

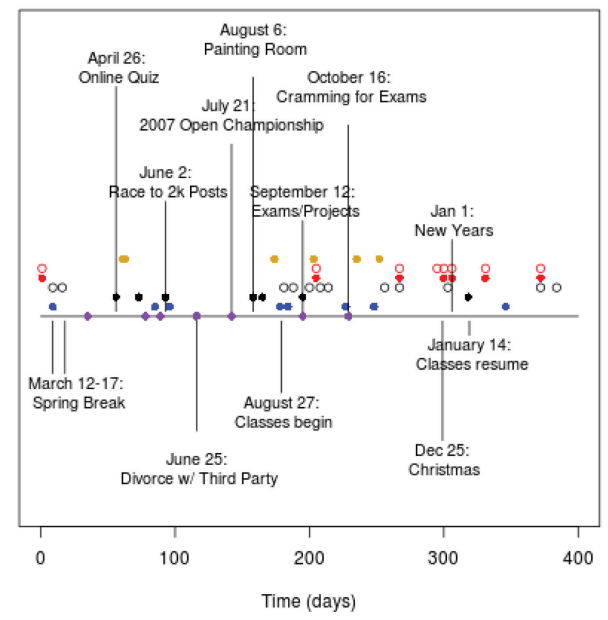

(c)

Fig. 18. Detected anomalies in (a) Enron corporation e-mail dataset, (b) university student e-mail dataset, and (c) Facebook wall postings dataset. Filled circles are detections from our proposed statistics, open circles are other methods.

Figure 18(b) shows the detected time steps of the University E-mail dataset. Several major events from the academic school year like the start of the school year and Christmas break are shown. It seems that the consistent statistics flag times closer to holidays and other events compared to other statistics. Unfortunately, as the text content of the messages was unavailable it is impossible to determine if the detected conversations correspond to specific events based on the dialogues of users.

Figure 18(c) shows the detected events of the Facebook wall data and the explanations for the detected events. Some of the listed events are holidays, while others were obtained by investigating the time steps flagged as anomalies; see Section 7 for an explanation of this process. Some events of interest are as follows: the "Race to $2 \mathrm{k}$ Posts" where a pair of individuals noticed they were nearing 


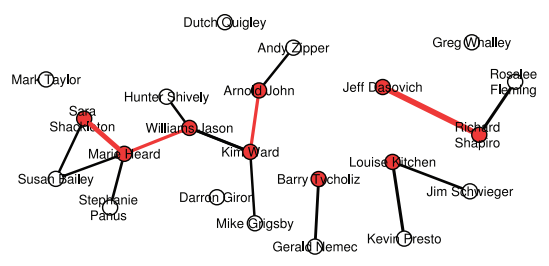

(a)

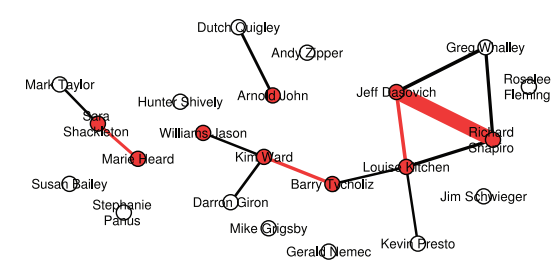

(b)

Fig. 19. Subgraph responsible for most of the mass shift anomaly in the Enron network at the weeks of June 25 (before anomaly) and July 2 (during anomaly), 2001, respectively.

two thousand posts on one of their walls and decided to reach that mark in one night, generating much more traffic between them than usual (over 160 posts); the "Divorce w/ Third Party" where a pair of individuals were going through a messy breakup and a mutual friend was cracking jokes and egging them on; and a discussion about Tiger Woods' odds in the 2007 Open Championship.

\section{LOCAL ANOMALY DECOMPOSITION}

After flagging a time step as anomalous it is useful to have some indication as to what is happening in the network at that time that generated the flag. One tool for investigating the flagged time step is local anomaly decomposition, where the network is broken down into subgraphs that contribute the most value to the total statistic score at that time step. For many statistics like mass shift or TP, which are summations over the edges, nodes, or triplets of the graph, this process is trivial: each component of the summation has an associated anomaly score and the components that provide the most anomaly score are the ones investigated. For others such as PDD, which cannot be easily decomposed into node and edge contributions, this approach is nearly impossible. Anomaly score decomposition is more useful when the score is skewed rather than uniformly distributed as it is easier to highlight a concise region that contributes the most toward the anomaly.

To demonstrate the decomposition, we applied the statistics to the real-world networks and sorted all of the nodes (for Barrat clustering) or edges (all other statistics) from highest to lowest contribution to the anomaly score sum. From there we selected the components with the highest anomaly score contribution totaling at least $20 \%$ of the log of the anomaly score to be part of the visualized anomaly. We then plotted all of the selected components as well as any adjacent edges and nodes. We investigated the Enron and Facebook datasets as these have names/message content associated with the graphs; the e-mail dataset has neither so these graphs are omitted.

Figures 19-22 show the local subgraphs reported by the mass shift, TP, GED, and Barrat clustering, respectively. The left subgraph shows activity in the time step immediately prior to the anomaly, while the right shows the subgraph during the anomaly. Red nodes and edges are part of the top anomaly contributors, while black edges and nodes are merely adjacent; the thickness of the edges corresponds to the edge weight in that time step.

Figure 19 shows an unusually large amount of communication between Senior Vice President Richard Shapiro and Government Relations Executive Jeff Dasovich immediately before Lay approaches Skilling about resigning as CEO. Figure 20 shows the triangular communications occurring between members of the Enron legal department which was occurring during the price-fixing strategy in California. Both of these methods find succinct subgraphs to represent the anomalies occurring at these times.

Figure 21, on the other hand, shows GED reporting nearly the entirety of the network at that time. While this does represent an event (the Congressional hearings) there is no interpretation 


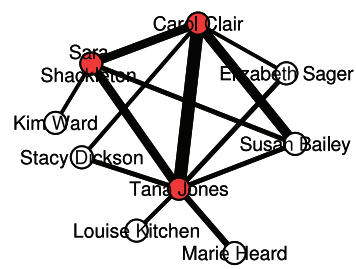

(a)

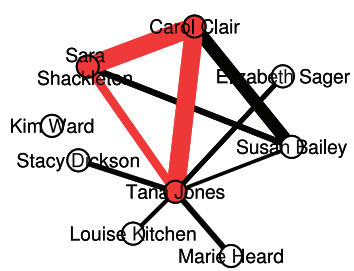

(b)

Fig. 20. Subgraph responsible for most of the triangle probability anomaly in the Enron network at the weeks of May 1 (before anomaly) and May 8 (during anomaly), 2000, respectively.

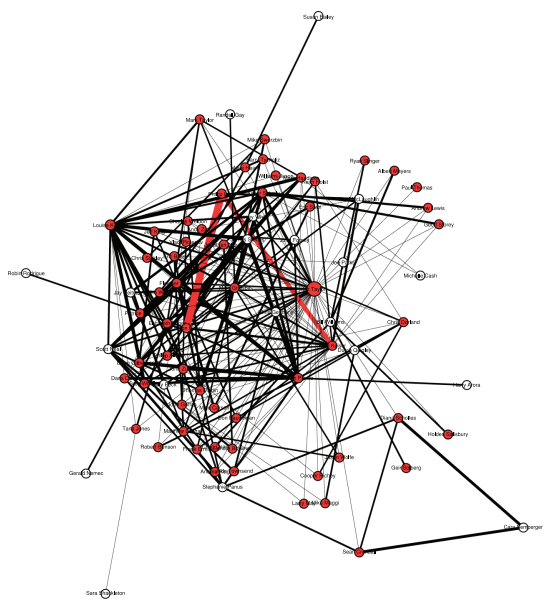

(a)

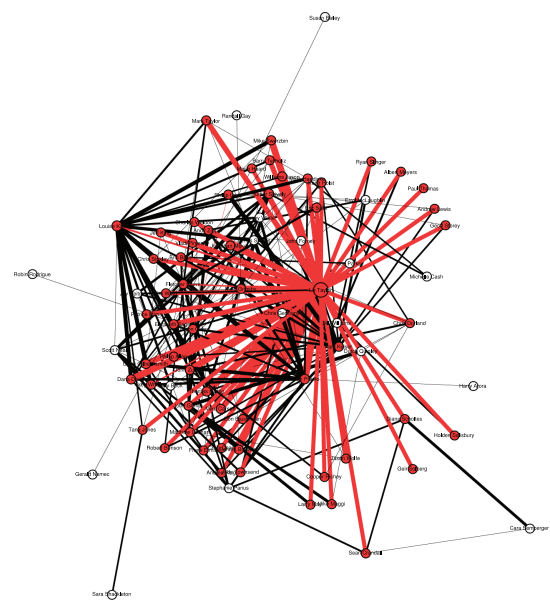

(b)

Fig. 21. Subgraph responsible for most of the graph edit distance anomaly in the Enron network at the weeks of January 28 (before anomaly) and Feburary 4 (during anomaly), 2002, respectively.

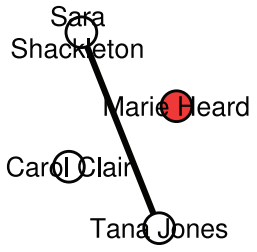

(a)

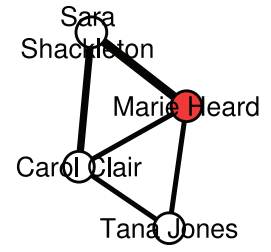

(b)

Fig. 22. Subgraph responsible for most of the Barrat clustering anomaly in the Enron network at the weeks of November 1 (before anomaly) and November 8 (during anomaly), 1999, respectively.

of the event other than that there were many messages being sent at that time. Barrat clustering identifies the legal department in Figure 22 but does so at a time with relatively low communication. Barrat clustering normalizes by node degree, which makes it more likely to report triangles with less weight as long as the participating nodes do not communicate with anyone else.

Figures 23-26 show the local subgraphs found in the Facebook dataset. Figure 23 shows the event we named "race to $2 \mathrm{k}$ posts," at this time a pair of individuals noticed they were closing 


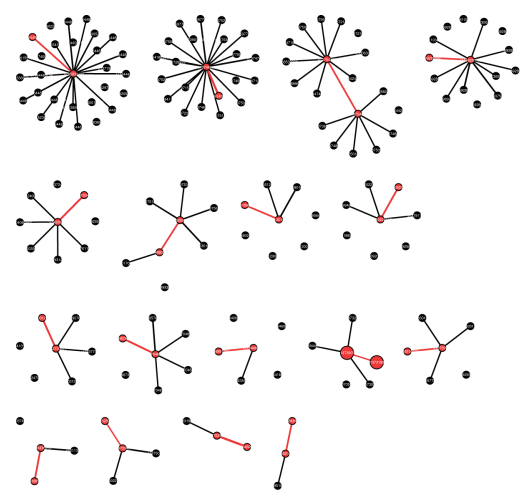

(a)

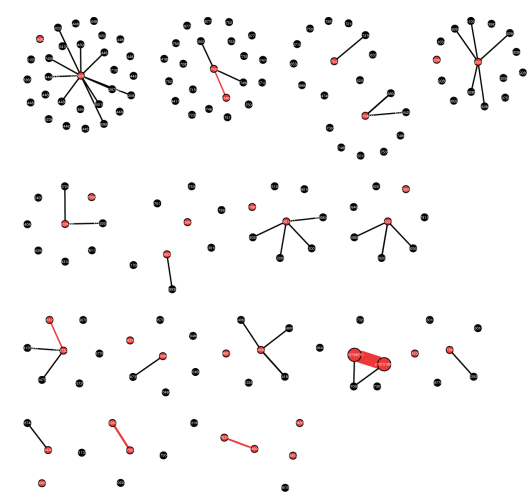

(b)

Fig. 23. Subgraph responsible for most of the mass shift anomaly in the Facebook network at June 1 (before anomaly) and June 2 (during anomaly), 2007, respectively.

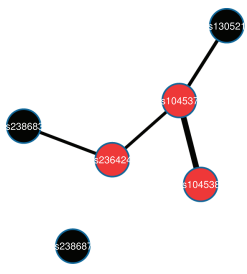

(a)

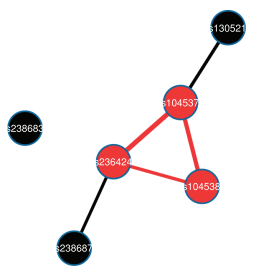

(b)

Fig. 24. Subgraph responsible for most of the triangle probability anomaly in the Facebook network at July 20 (before anomaly) and July 21 (during anomaly), 2007, respectively.

in on two thousand posts on their walls and decided to reach that goal in one night. The result is a massively higher amount of communication than was typical between the two in prior time steps. Figure 24 shows the communications occurring during the 2007 Open Championship golf tournament. The three individuals with the most communication were arguing about the odds that Tiger Woods would win the tournament.

GED, by contrast, identifies no coherent local structure in Figure 25. It is likely that this event signifies a global increase in communication rather than a change in the distribution of messages. As the additional edges were distributed throughout the network, when looking for subgraphs that generated the most anomaly score the majority of the network has similar scores so a random chunk of the network is found. Figure 26 is the structure found by Barrat clustering; as before it finds a set of triangular communication with relatively low weights, around $2-4$, while the anomaly found by TP has about 12 messages per edge.

\section{RELATED WORK}

The scope of papers related to this work and their relationship with each other are categorized in Tables 4-7. The four dimensions of the papers categorized in the tables are as follows: (1) the network property being tested or the goal of the hypothesis test in the paper; (2) whether the graphs being tested are dynamic or static; (3) the type of test statistic used; and (4) the method 


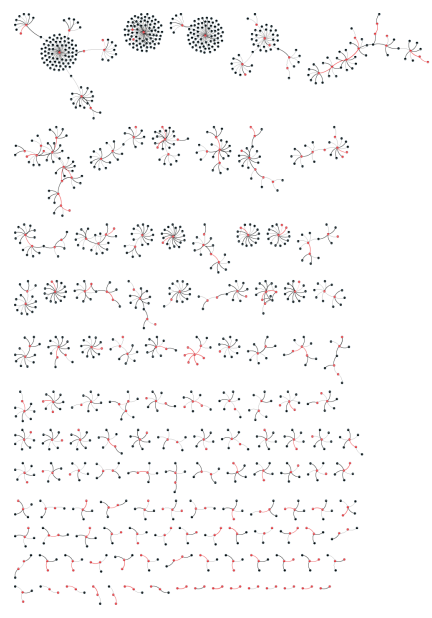

(a)

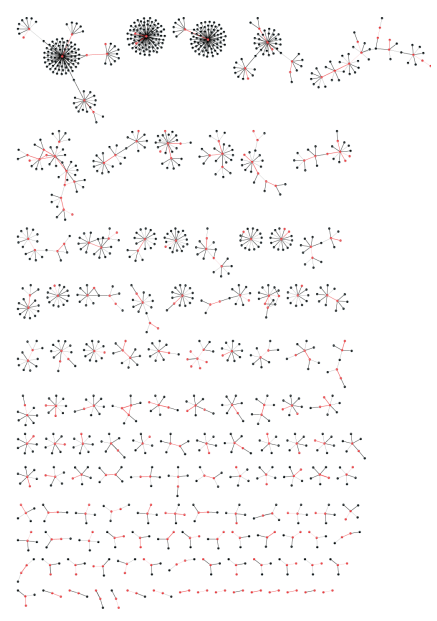

(b)

Fig. 25. Subgraph responsible for most of the graph edit distance anomaly in the Facebook network at October 15 (before anomaly) and October 16 (during anomaly), 2007, respectively.

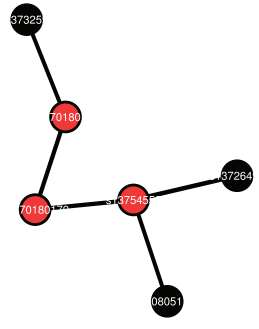

(a)
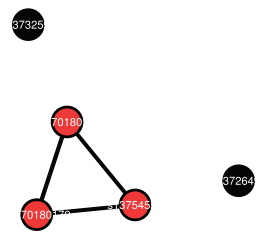

(b)

Fig. 26. Subgraph responsible for most of the Barrat clustering anomaly in the Facebook network at May 17 (before anomaly) and May 18 (during anomaly), 2007, respectively.

used to construct or estimate the null distribution. All of the tables have network property as a dimension. Tables 4 and 5 show the type of test statistic for static and dynamic graphs, respectively, while Tables 6 and 7 show the method of null distribution generation for static and dynamic graphs.

The network property and dynamic/static nature of the data are inherent to the problem definition of the papers, while the test statistic and null distribution construction are algorithmic choices and could theoretically be replaced with another approach. As the test statistic and null distribution construction are arranged vertically in the tables papers in the same columns are tackling the same kinds of problems and can be directly compared to one another.

Model parameters and likelihood both refer to model-based hypothesis testing, while distance measure, basic statistic, and network statistic are all statistic-based approaches. Distance Measure refers exclusively to delta statistics evaluating graph difference. Basic Statistics consists of simple statistics like edge count, node count, or the number of nodes with a particular attribute, while Network Statistic has more complicated statistics like autocorrelation or clustering coefficient. 
Table 4. Categorization of Related Work in Static Networks, by Type of Statistic and Network Property

\begin{tabular}{|c|c|c|c|c|}
\hline \multirow[b]{2}{*}{ Statistic type } & \multicolumn{4}{|c|}{ Network property } \\
\hline & $\begin{array}{c}\text { Distinguish } \\
\text { graphs }\end{array}$ & $\begin{array}{l}\text { Global } \\
\text { property }\end{array}$ & $\begin{array}{c}\text { Community } \\
\text { property }\end{array}$ & $\begin{array}{c}\text { Local } \\
\text { property }\end{array}$ \\
\hline Model parameters & {$[19]$} & {$[15,26,30]$} & {$[26]$} & \\
\hline Likelihood & {$[19,36,37]$} & & [23] & [51] \\
\hline Distance measure & {$[2,17,52]$} & {$[2,26]$} & & \\
\hline Basic statistic & [26] & {$[26,45,53]$} & & \\
\hline Network statistic & {$[2,17,52]$} & [26] & {$[2,12,21,26,27]$} & {$[2,21,26,27,50,54]$} \\
\hline
\end{tabular}

Table 5. Categorization of Related Work in Dynamic Networks, by Type of Statistic and Network Property

\begin{tabular}{|l|c|c|c|c|c|}
\hline & \multicolumn{5}{|c|}{ Network property } \\
\cline { 2 - 6 } Statistic type & $\begin{array}{c}\text { Distinguish } \\
\text { graphs }\end{array}$ & $\begin{array}{c}\text { Evolution } \\
\text { property }\end{array}$ & $\begin{array}{c}\text { Global } \\
\text { property }\end{array}$ & $\begin{array}{c}\text { Community } \\
\text { property }\end{array}$ & $\begin{array}{c}\text { Local } \\
\text { property }\end{array}$ \\
\hline $\begin{array}{l}\text { Model parameters } \\
\text { Likelihood }\end{array}$ & {$[14]$} & {$[11,16,18,46,48,49]$} & & \\
Distance measure & {$[2,7,29,31,39]$} & {$[42]$} & {$[25]$} & & \\
Basic statistic & {$[24]$} & {$[10,11]$} & {$[11,45]$} & & {$[11]$} \\
Network statistic & {$[2,7,31,34,35,41,44]$} & & {$[2,4,11,18,20,34]$} & {$[20]$} & {$[11,33]$} \\
\hline
\end{tabular}

Table 6. Categorization of Related Work in Static Networks, by Null Distribution $\left(H_{0}\right)$

Generation Method and Network Property

\begin{tabular}{|l|c|c|c|c|}
\hline & \multicolumn{4}{|c|}{ Network property } \\
\cline { 2 - 5 }$H_{0}$ Generation Method & $\begin{array}{c}\text { Distinguish } \\
\text { graphs }\end{array}$ & $\begin{array}{c}\text { Global } \\
\text { property }\end{array}$ & $\begin{array}{c}\text { Community } \\
\text { property }\end{array}$ & $\begin{array}{c}\text { Local } \\
\text { property }\end{array}$ \\
\hline Multiple observations & {$[19]$} & {$[34]$} & & {$[26,50,54]$} \\
Generative model & {$[36,37]$} & {$[53]$} & & \\
Sampling method & {$[26]$} & {$[26,45]$} & & \\
Permutation & {$[17,52]$} & {$[15,26,30]$} & {$[12,26]$} & {$[26]$} \\
\hline
\end{tabular}

Some of the cited papers in the tables are surveys that cover a wide variety of methods. Look to these for general information about Social Network Analysis [10, 26], Anomaly Detection [2], or Homophily and Social Influence [5].

The work described in the current article is represented in the tables with the citation [31], which is a reference to a preliminary work. The current article introduces new consistent network statistics that aid in determining which graph instances have anomalous network properties when the size of those graphs is not constant. It utilizes both statistics measuring single graphs and distance measures comparing pairs of graphs and therefore falls in both Network statistic and distance measure rows, and it constructs the null distribution using multiple observations. As it seeks to differentiate graphs based on their network properties (and whether those properties are anomalous), it falls in the distinguish graphs category.

The two most relevant papers to the current work are the ones describing the Netsimile [7] and Deltacon [29]. Both of these statistics are distance measures designed to find graphs that are different from normal observed graphs. Netsimile accomplishes this by creating vectors for each 
Table 7. Categorization of Related Work in Dynamic Networks, by Null Distribution $\left(H_{0}\right)$ Generation Method and Network Property

\begin{tabular}{|l|c|c|c|c|c|}
\hline \multirow{2}{*}{$H_{0}$ Generation method } & \multicolumn{4}{|c|}{ Network property } \\
\cline { 2 - 6 } & $\begin{array}{c}\text { Distinguish } \\
\text { graphs }\end{array}$ & $\begin{array}{c}\text { Evolution } \\
\text { property }\end{array}$ & $\begin{array}{c}\text { Global } \\
\text { property }\end{array}$ & $\begin{array}{c}\text { Community } \\
\text { property }\end{array}$ & $\begin{array}{c}\text { Local } \\
\text { property }\end{array}$ \\
\hline Multiple observations & {$[2,7,29,31,34,35]$} & {$[14,42]$} & {$[16,46,48,49]$} & & {$[33]$} \\
Generative model & {$[24,39,41,44]$} & & & & \\
Sampling method & & & {$[10]$} & & \\
Permutation & & & {$[4,25,45]$} & & \\
\hline
\end{tabular}

graph out of aggregates of network statistics and then taking the distance between those summary vectors, while Deltacon calculates a matrix for each graph representing distances between nodes and then compares those matrices. These statistics are used as a basis for comparison as they are both state-of-the-art detectors for dynamic network anomalies. In addition, Deltacon explicitly searches for anomalies on a dataset, which this work also investigates.

Other papers that search for dynamic network anomalies using more traditional statistics include the following: [13, 24, 35, 39, 41, 44, 50]. Model-based anomaly detection is also common, the most used model being ERGM: [6, 16, 19, 25, 34, 46, 47, 49,52], although some use other types of models [23, 36, 51].

Another paper that delves into the statistical consistency properties of network statistics is Borgs et al. [9], which investigates the behavior of motif-style statistics in the limit as the number of nodes sampled from some graph generative process increases. Similar to our approach they prove a finite limit that depends only on the properties of the graph generative process. However, they only take the limit with respect to the number of nodes, making the assumption that once a pair nodes are both in the observed set, the true edge weight between them is known and not estimated from network communications.

\section{CONCLUSIONS}

In this article, we have discussed one of the fundamental problems facing anomaly detection in dynamic networks: handling changes to the amount of individuals and the quantity of communications that they produce. We have shown that naively applying previously used statistics to this domain lead to incorrect conclusions due to these message count and node count variations. To remedy this problem, we have introduced the property of Size Consistency for network statistics and shown theoretically how this property ensures accurate detection of changes or anomalies in dynamic networks.

We proposed three Size Consistent network statistics, Mass Shift, Degree Shift, and TP to replace the previously used statistics of GED, Degree Distribution, and Clustering Coefficient. These statistics are proven to be Size Consistent and we demonstrated using synthetic and semi-synthetic experiments that anomaly detectors using our statistics have superior performance when detecting known anomalies in a dynamic network where the message and edge count vary.

We also applied these new statistics to a number of real-world networks to see what kind of anomalous events would be detected. In addition to showing that the Size Consistent statistics are less prone to flagging time steps that are unusual in message or node count only, we analyzed the location in the network that was producing the detected anomalies. We found that when searching for local explanations for detected anomalies that Size Consistent statistics produce more concise and interpretable explanations than conventional statistics. 
The framework for developing Size Consistent network statistics can be applied to new statistics in the future. We hope that researchers who propose network statistics in the future will make sure to analyze the effects that changing network size have on their proposed statistics and ensure that those statistics meet the Size Consistency requirements.

\section{A APPENDIX}

\section{A.1 Proof of Theorem 3.1}

Proof. If $\left\{G_{1} \ldots G_{x}\right\}$ and $G_{t e s t}$ are drawn from $P^{* n}$, then $\widehat{S}_{k}\left(G_{1}\right) \ldots \widehat{S}_{k}\left(G_{x}\right)$ and $\widehat{S}_{k}\left(G_{\text {test }}\right.$ are all converging to $S_{k}\left(P^{* n}\right)$ as the same distribution of values and the hypothesis test will reject with the $p$-value probability of $\alpha$.

\section{A.2 Proof of Theorem 3.2}

Proof. If $\widehat{S}_{k}$ is a Size Consistent statistic, then $\widehat{S}_{k}\left(G_{\text {test }}\right) \rightarrow S_{k}\left(P^{* a}\right)$ and $\widehat{S}_{k}\left(G_{1}\right), \widehat{S}_{k}\left(G_{2}\right), \ldots \widehat{S}_{k}\left(G_{x}\right) \rightarrow S_{k}\left(P^{* n}\right)$ as $\left|W_{\text {min }}\right| \rightarrow \infty$ and $\left|V_{\text {min }}\right| \rightarrow\left|V^{*}\right|$. If $S_{k}\left(P^{* a}\right) \neq S_{k}\left(P^{* n}\right)$, then the statistic $\widehat{S}_{k}\left(G_{\text {test }}\right)$ and the set of statistics $\widehat{S}_{k}\left(G_{1}\right), \widehat{S}_{k}\left(G_{2}\right), \ldots \widehat{S}_{k}\left(G_{x}\right)$ converge to different values and $G_{\text {test }}$ will be flagged as an anomaly with probability 1 .

\section{A.3 Proof of Theorem 3.3}

Proof. When the set of graphs $\left\{G_{1} \ldots G_{x}\right\}$ are used to estimate an empirical distribution of the null $\widehat{S_{k}}$, the distribution is bounded by $\max \left[S_{k}\left(\left\{G_{1} \ldots G_{x}\right\}\right)\right]$ and $\min \left[S_{k}\left(\left\{G_{1} \ldots G_{x}\right\}\right)\right]$, so the critical points $\phi_{\text {lower }}$ and $\phi_{\text {upper }}$ of a hypothesis test using this set of graphs will be within these bounds. Since an increasing $\left|W_{\text {test }}\right|$ or $\left|V_{\text {test }}\right|$ implies $S_{k}\left(G_{\text {test }}\right)$ diverges to infinity, then there exists a $\left|W_{\text {test }}\right|$ or $\left|V_{\text {test }}\right|$ such that $S_{k}\left(G_{\text {test }}\right)$ is not within $\phi_{\text {lower }}$ and $\phi_{\text {upper }}$ and will be rejected by the test.

\section{A.4 Proof of Theorem 3.4}

Proof. Let $G_{\text {test }}$ be the test network drawn from $P^{* a}, M_{W}^{n}$, and $M_{V}^{n}$, and $\left\{G_{1} \ldots G_{x}\right\}$ be the set of null distribution graphs drawn from $P^{* n}, M_{W}^{n}$, and $M_{V}^{n}$. If $S_{k}(G)$ is a divergent function of $|W|$ or $|V|$, then the variance of the null distribution $\widehat{S}_{k}$ estimated from $\left\{G_{1} \ldots G_{x}\right\}$ is dependent on the variance of $|W|$ and $|V|$. If the variance of sampled $|W|$ or $|V|$ is sufficiently large, the variance of $\widehat{S}_{k}$ will increase to cover all possible $S_{k}(G)$ values, and the test instance will fail to be flagged as an anomaly with probability $1-\alpha$.

\section{A.5 Proof of Theorem 3.5}

Proof. Let $M_{W}^{n}, M_{V}^{n}$ be such that $\widehat{S}_{k}\left(G_{x}, G_{y}\right)$ is approaching $S_{1}$ for any pair of graphs from $\left\{G_{1} \ldots G_{d}\right\}$ as $\left|W_{\text {min }}\right| \rightarrow \infty$ and $\left|V_{\text {min }}\right| \rightarrow V^{*}$. Likewise, let $M_{W}^{a}, M_{V}^{a}$ be such that $\widehat{S}_{k}\left(G_{t e s t, 1}, G_{t e s t, 2}\right)$ is approaching $S_{2}$. For sufficiently large $\left|W_{\text {min }}\right|,\left|V_{\text {min }}\right|$ the bias of these statistics from their limits is 0 , and since $S_{1} \neq S_{2}$ the probability of a false positive is 1 .

\section{A.6 Proof of Theorem 3.6}

Proof. Let $M_{W}^{n}$ and $M_{V}^{n}$ be such that the value of $\widehat{S}_{k}$ is close to either $S_{u p p e r}^{n}$ or $S_{\text {lower }}^{n}$ on graphs $\left\{G_{1} \ldots G_{X}\right\}$ and close to $S^{a}$ for $\widehat{S}_{k}\left(G_{t e s t}, G_{x}\right)$. Additionally let the probability of converging to $S_{u p p e r}^{n}$ or $S_{\text {lower }}^{n}$ on data drawn from $P^{* n}, M_{W}^{n}, M_{V}^{n}$ be such that the mean of the null distribution is equal to $S^{a}$. Then, as $\left|W_{\text {min }}\right| \rightarrow \infty$ and $\left|V_{\text {min }}\right| \rightarrow\left|V^{*}\right|$ the mean of the null distribution is close to $S^{a}$ and the probability of producing a false negative goes to 1 . 


\section{A.7 Proof of Claim 4.1}

Edit Distance is a Size Inconsistent statistic.

Proof. Consider the case where $G_{1}$ and $G_{2}$ are both drawn from $P^{*}$. Let $\left|W_{2}\right|=\left|W_{1}\right|+W_{\Delta}$. The expected difference in weights between two nodes $i, j$ in $G_{1}$ versus $G_{2}$ is

$$
E\left[w_{i j, 1}-w_{i j, 2}\right]=\left|W_{1}\right| p_{i j}-\left(\left|W_{1}\right|+W_{\Delta}\right) p_{i j}=W_{\Delta} p_{i j} .
$$

As $W_{\Delta}$ increases the expected difference in the edge weights goes to infinity, which causes the GED to go to infinity. As the statistic has at least one infinite limit it is a Divergent Statistic and the conditions of Size Consistency are violated.

\section{A.8 Proof of Claim 4.2}

Raw Degree is a Size Inconsistent statistic.

Proof. If the Degree Distance is Size Consistent, then the $\operatorname{limit}_{\left(\left|W_{1}\right|,\left|W_{2}\right|\right) \rightarrow(\infty, \infty)} D D\left(G_{1}, G_{2}\right)$ must have a single multivariate limit that is finite.

Let $\left|W_{1}\right|=\left|W_{2}\right|=|W|$. Then, the limit becomes

$$
\lim _{|W| \rightarrow \infty} \sum_{d \in\left[1, d_{\text {max }}\right]} a b s\left(\sum_{i \in V_{1}} I\left[\sum_{j \in V_{1}} w_{i j, 1}=d\right]-\sum_{i \in V_{2}} I\left[\sum_{j \in V_{2}} w_{i j, 2}=d\right]\right) .
$$

As $w_{i j, x}$ is a binomial variable, the limit on $\sum_{i \in V_{x}} w_{i j, x}$ as $\left|W_{x}\right|$ goes to infinity is $\sum_{i \in V_{x}}\left|W_{x}\right| p_{i j, x}$ by the law of large numbers. This makes the limit

$$
\lim _{|W| \rightarrow \infty} \sum_{d \in\left[1, d_{\text {max }}\right]} a b s\left(\sum_{i \in V_{1}} I\left[\sum_{j \in V_{1}}|W| p_{i j, 1}=d\right]-\sum_{i \in V_{2}} I\left[\sum_{j \in V_{2}}|W| p_{i j, 2}=d\right]\right) .
$$

Define $p_{\text {row }}$ such that $|W| p_{\text {row }}=d$. We can now rewrite the limit as

$$
\begin{gathered}
\lim _{|W| \rightarrow \infty} \sum_{p \in P_{\text {row }}} a b s\left(\sum_{i \in V_{1}} I\left[\sum_{j \in V_{1}}|W| p_{i j, 1}=|W| p\right]-\sum_{i \in V_{2}} I\left[\sum_{j \in V_{2}}|W| p_{i j, 2}=|W| p\right]\right) \\
=\sum_{p \in P_{\text {row }}} a b s\left(\sum_{i \in V_{1}} I\left[\sum_{j \in V_{1}} p_{i j, 1}=p\right]-\sum_{i \in V_{2}} I\left[\sum_{j \in V_{2}} p_{i j, 2}=p\right]\right),
\end{gathered}
$$

where $P_{\text {row }}$ is the set of all unique row summations in $P_{1}$ and $P_{2}$.

Now, let $\left|W_{2}\right|=\left|W_{1}\right|+\left|W_{1}\right| \frac{p_{\max 1}}{p_{\min 2}}$ where $p_{\max 1}$ is the maximum value of any row in $P_{1}$ and $p_{\min 2}$ is the minimum non-zero value in rows of $P_{2}$. As $\left|W_{1}\right| \rightarrow \infty$, the minimum value of any row in $W_{2}$ becomes $\left|W_{1}\right| p_{\min 2}+\left|W_{1}\right| p_{\max 1}$ while the maximum value of any row in $W_{1}$ becomes $\left|W_{1}\right| p_{\max 1}$. This guarantees that in the limit no nodes in $G_{1}$ will have the same degree as a node in $G_{2}$, allowing us to rewrite the limit as

$$
\begin{aligned}
& \lim _{\left|W_{1}\right| \rightarrow \infty} \sum_{d \in\left[1, d_{\text {max }}\right]}\left(\sum_{i \in V_{1}} I\left[\sum_{j \in V_{1}}\left|W_{1}\right| p_{i j, 1}=d\right]+\sum_{i \in V_{2}} I\left[\sum_{j \in V_{2}}\left|W_{1}\right| p_{i j, 2}=d\right]\right) \\
& =\left|V_{1}\right|+\left|V_{2}\right| .
\end{aligned}
$$

Because we have found the statistic converging to multiple values, no multivariate limit exists for the Degree Distance as $\left(\left|W_{1}\right|,\left|W_{2}\right|\right) \rightarrow(\infty, \infty)$ and the statistic is Size Inconsistent. 


\section{A.9 Proof of Claim 4.3}

Barrat Clustering is a Size Consistent statistic.

Proof. First, we will find $C B(P)$ by taking the limit as $|W| \rightarrow \infty$. As $w_{i j} \rightarrow|W| p_{i j}$ as $|W| \rightarrow \infty$, $\widehat{a_{i j}} \rightarrow a_{i j}$ for any $i, j$, where $a_{i j}=I\left[p_{i j}>0\right]$. Similarly, we can replace $D_{i}(G)$ with $|W| \sum_{j} p_{i j}$. This lets us rewrite the limit as

$$
\begin{aligned}
\lim _{|W| \rightarrow \infty} C B(G) & =\lim _{|W| \rightarrow \infty} \sum_{i} \frac{1}{|V|\left(\widehat{a}_{i}-1\right) D_{i}(G)} \sum_{j, k} \frac{w_{i j}+w_{i k}}{2} \widehat{a}_{i j} \widehat{a}_{i k} \widehat{a}_{j k} \\
& =\sum_{i} \frac{1}{|V|\left(a_{i}-1\right)|W| \sum_{j} p_{i j}} \sum_{j, k} \frac{|W|\left(p_{i j}+p_{i k}\right)}{2} a_{i j} a_{i k} a_{j k} \\
& =\sum_{i} \frac{1}{|V|\left(a_{i}-1\right) \sum_{j} p_{i j}} \sum_{j, k} \frac{\left(p_{i j}+p_{i k}\right)}{2} a_{i j} a_{i k} a_{j k} \\
& =C B(P) .
\end{aligned}
$$

Now, we take the $\operatorname{limit}_{|V| \rightarrow\left|V^{*}\right|} C B(P)$. Similar to before, $a_{i j} \rightarrow a_{i j}^{*}$ :

$$
\begin{aligned}
\lim _{|V| \rightarrow\left|V^{*}\right|} C B(P) & =\lim _{|V| \rightarrow\left|V^{*}\right|} \sum_{i} \frac{1}{|V|\left(a_{i}-1\right) \sum_{j} p_{i j}} \sum_{j, k} \frac{\left(p_{i j}+p_{i k}\right)}{2} a_{i j} a_{i k} a_{j k} \\
& =\lim _{|V| \rightarrow\left|V^{*}\right|} \sum_{i} \frac{1}{|V|\left(a_{i}-1\right) \frac{1}{\sum_{i j \in V} p_{i j}^{*}} \sum_{j} p_{i j}^{*}} \sum_{j, k} \frac{1}{\sum_{i j \in V} p_{i j}^{*}} \frac{\left(p_{i j}^{*}+p_{i k}^{*}\right)}{2} a_{i j} a_{i k} a_{j k} \\
& =\sum_{i} \frac{1}{\left|V^{*}\right|\left(a_{i}^{*}-1\right) \sum_{j} p_{i j}^{*}} \sum_{j, k} \frac{\left(p_{i j}^{*}+p_{i k}^{*}\right)}{2} a_{i j}^{*} a_{i k}^{*} a_{j k}^{*} .
\end{aligned}
$$

As Barrat Clustering has a finite limit as $|W| \rightarrow \infty$ and $|V| \rightarrow\left|V^{*}\right|$ it is a Size Consistent Statistic.

\section{A.10 Proof of Claim 4.4}

The error of the Barrat Clustering is bounded by

$$
\frac{3}{2\left|V^{*}\right|}
$$

and the probability that it achieves this bound is less than or equal to $1-\left(\begin{array}{c}\left|V^{*}\right|-3 \\ |V|-3\end{array}\right) /\left(\begin{array}{c}\left|V^{*}\right| \\ |V|\end{array}\right)$, making the statistic Node Constant.

Proof. The worst-case scenario in terms of maximum error and probability of attaining that maximum error occurs when most of the edge weight lies on a single triangle. Consider a graph with $\left|V^{*}\right|$ nodes consisting of three "outlier" nodes with the rest of the nodes being "normal." Normal nodes are arranged in pairs with an edge weight of 1 between them (as nodes without any edges are not considered part of the graph), while all remaining edge weight $\left(W-\frac{\left|V^{*}\right|-3}{2}\right)$ is evenly distributed between the three outlier nodes. If the Barrat clustering is calculated on a subset $V$ of the nodes, which does not contain all three of the outliers, the score will be 0 , while the true score 
is

$$
\begin{aligned}
B C(G) & =\sum_{i \in V} \frac{1}{|V|\left(a_{i}-1\right) D_{i}} \sum_{j k \in V} \frac{w_{i j}+w_{i k}}{2} a_{i j} a_{j k} a_{i k} \\
& =\frac{3}{\left|V^{*}\right| \frac{\left(2 W-\left|V^{*}\right|+3\right)}{3}} \frac{2 W-\left|V^{*}\right|+3}{6} \\
& =\frac{3}{2\left|V^{*}\right|} .
\end{aligned}
$$

Which is also the maximum error. As this error occurs whenever at least one of the outlier nodes is not in the set $V$, the probability of reaching the maximum error is at most $1-\left(\begin{array}{c}\left|V^{*}\right|-3 \\ |V|-3\end{array}\right) /\left(\begin{array}{c}\left|V^{*}\right| \\ |V|\end{array}\right)$.

\section{A.11 Proof of Claim 4.5}

\section{Deltacon is a Size Consistent statistic.}

Proof. The root Euclidean distance function $\operatorname{Dist}\left(S_{1}, S_{2}\right)$ is an algebraic function over the domain of non-negative input matrices. As the affinity matrices $S_{1}, S_{2}$ are guaranteed non-negative, this distance function is continuous and we merely need to show that

$$
\operatorname{plim}_{\left(\left|W_{1}\right|,\left|W_{2}\right|\right) \rightarrow(\infty, \infty)}\left(S_{1}, S_{2}\right)
$$

exists and is finite by Lemma 3.1.

We can simplify this by reducing to a single limit: $\operatorname{plim}_{\left|W_{1}\right| \rightarrow \infty} S_{1}$. Let $\widehat{D}_{1}$ be the unweighted diagonal degree matrix and $\widehat{A}$ be the adjacency matrix of $G_{1}$. Let $P_{1}$ be the probability matrix from which $G_{1}$ is sampled, which correspondingly has matrices $D_{1}$ and $A_{1}$, where $A_{1}$ is the non-zero cells of $P_{1}$.

Then, the limit

$$
\operatorname{plim}_{\left|W_{1}\right| \rightarrow \infty} S_{1}=\operatorname{plim}_{\left|W_{1}\right| \rightarrow \infty}\left[I+\epsilon^{2} \widehat{D}_{x}-\epsilon \widehat{A}_{x}\right]^{-1} \rightarrow\left[I+\epsilon^{2} D_{x}-\epsilon A_{x}\right]^{-1} .
$$

With infinite message samples any non-zero probability in $P_{1}$ will be sampled, ensuring that $\widehat{A}_{1} \rightarrow$ $A_{1}$ and $\widehat{D}_{1} \rightarrow D_{1}$. The proof for $S_{2}$ is identical. And as $S_{1}, S_{2}$ have finite limits, Deltacon has a finite multivariate limit as well.

Now, we need to show that $\lim _{\left(\left|V_{1}\right|,\left|V_{2}\right|\right) \rightarrow\left(\left|V^{*}\right|,\left|V^{*}\right|\right)} \operatorname{Deltacon}\left(P_{1}, P_{2}\right)$ exists and is finite.

As Deltacon is a summation over the nodes that exist in both graphs, we can replace $V_{1}, V_{2}$ with $V_{\cup}=V_{1} \cup V_{2}$, which approaches size $\left|V^{*}\right|$ as $\left(\left|V_{1}\right|,\left|V_{2}\right|\right) \rightarrow\left|V^{*}\right|$. The root Euclidean distance is a continuous function, so we only need to show that $S_{1}$ and $S_{2}$ have finite limits.

Let $P_{1}^{*}, P_{2}^{*}$ be the edge probabilities across nodes $V^{*}$, and let $A_{1}^{*}, A_{2}^{*}$ be their adjacency matrices and $D_{1}^{*}, D_{2}^{*}$ be the diagonal unweighted degree matrices. As $V_{x} \rightarrow V^{*}, A_{x} \rightarrow A_{x}^{*}$, and $D_{x} \rightarrow D_{x}^{*}$ as every node in $V^{*}$ is eventually included. This guarantees that

$$
\lim _{\left(\left|V_{1}\right|,\left|V_{2}\right|\right) \rightarrow\left(\left|V^{*}\right|,\left|V^{*}\right|\right)} S_{1}=\left[I+\epsilon^{2} D_{x}^{*}-\epsilon A_{x}^{*}\right]^{-1} .
$$

As Deltacon has finite multivariate limits for both $\left|W_{1}\right|,\left|W_{2}\right|$ and $\left|V_{1}\right|,\left|V_{2}\right|$, it is a Size Consistent statistic.

Proof of Claim 4.6: The maximum error of Deltacon is $\epsilon_{\max } \geq \sqrt{c}\left(\left|V^{*}\right|-\left|V_{1} \cup V_{2}\right|\right)$ for some constant $c$. 
Proof. Deltacon is defined as

$$
D C\left(S_{1}, S_{2}\right)=\sqrt{\sum_{i, j \in V_{1} \cup V_{2}}\left(\sqrt{s_{i j, 1}}-\sqrt{s_{i j, 2}}\right)^{2}},
$$

where $S_{x}=\left[I+\epsilon^{2} D_{x}-\epsilon A_{x}\right]^{-1}$ is the "affinity matrix" where larger positive values represent greater proximity in the graph.

Assume that the observed edges and nodes are identical in both time steps: $E_{1}=E_{2}, V_{1}=V_{2}$. Also assume that in time 1 the remaining nodes of $V^{*}$ all have a path to any node in $V_{1} \cup V_{2}$ but that no non-overlapping path exists between a pair of nodes in $V_{1} \cup V_{2}$ that includes a node from the unsampled set $V^{*}-\left(V_{1} \cup V_{2}\right)$, while in time 2 the remaining nodes have no edges at all. This gives us a maximum error of

$$
\begin{aligned}
\epsilon_{\max } & =\sqrt{\sum_{i, j \in V^{*}}\left(\sqrt{s_{i j, 1}}-\sqrt{s_{i j, 2}}\right)^{2}} \\
& =\sqrt{\sum_{i \in V_{1} \cup V_{2}, j \in V^{*}-\left(V_{1} \cup V_{2}\right)}\left(\sqrt{s_{i j, 1}}\right)^{2}+\sum_{j \in V_{1} \cup V_{2}, i \in V^{*}-\left(V_{1} \cup V_{2}\right)}\left(\sqrt{s_{i j, 1}}\right)^{2}+\sum_{i, j \in V^{*}-\left(V_{1} \cup V_{2}\right)}\left(\sqrt{s_{i j, 1}}\right)^{2}} \\
& =\sqrt{\sum_{i \in V_{1} \cup V_{2}, j \in V^{*}-\left(V_{1} \cup V_{2}\right)} s_{i j, 1}+\sum_{j \in V_{1} \cup V_{2}, i \in V^{*}-\left(V_{1} \cup V_{2}\right)} s_{i j, 1}+\sum_{i, j \in V^{*}-\left(V_{1} \cup V_{2}\right)} s_{i j, 1}}
\end{aligned}
$$

For simplicity let us bound this with

$$
\epsilon_{\max } \geq \sqrt{\sum_{i, j \in V^{*}-\left(V_{1} \cup V_{2}\right)} s_{i j, 1}}
$$

If the nodes of $V^{*}-\left(V_{1} \cup V_{2}\right)$ are completely connected to each other, $s_{i j, 1}>c$ for some constant $c$ :

$$
\begin{aligned}
& \epsilon_{\text {max }} \geq \sqrt{\sum_{i, j \in V^{*}-\left(V_{1} \cup V_{2}\right)} c} \\
& \epsilon_{\text {max }} \geq \sqrt{c\left(\left|V^{*}\right|-\left|V_{1} \cup V_{2}\right|\right)^{2}} \\
& \epsilon_{\text {max }} \geq \sqrt{c}\left(\left|V^{*}\right|-\left|V_{1} \cup V_{2}\right|\right) .
\end{aligned}
$$

As the maximum error is at least a function of $\left|V^{*}\right|-\left|V_{1} \cup V_{2}\right|$, the statistic is Pseudo-Diverging.

\section{A.12 Proof of Claim 4.7}

Weighted Netsimile is a Size Inconsistent statistic.

Proof. If $N S\left(G_{1}, G_{2}\right)$ is Size Consistent then a single finite limit $L$ must exist for the multivariate limit $\lim _{\left(\left|W_{1}\right|,\left|W_{2}\right|\right) \rightarrow(\infty, \infty)} N S\left(G_{1}, G_{2}\right)$.

Define $N S^{\prime}\left(G_{1}, G_{2}\right)$ to be a simplified version of Netsimile that uses only one node feature, weighted node degree, and one aggregator, the mean of all nodes. Note that this statistic is one component of the sum across all elements in $\vec{s}$, and so $N S^{\prime}\left(G_{1}, G_{2}\right)<N S\left(G_{1}, G_{2}\right)$. 
The $\operatorname{limit}_{\left(\left|W_{1}\right|,\left|W_{2}\right|\right) \rightarrow(\infty, \infty)} N S^{\prime}\left(G_{1}, G_{2}\right)$ can be written as

$$
\begin{aligned}
\lim _{\left(\left|W_{1}\right|,\left|W_{2}\right|\right) \rightarrow(\infty, \infty)} N S^{\prime}\left(G_{1}, G_{2}\right) & =\lim _{\left(\left|W_{1}\right|,\left|W_{2}\right|\right) \rightarrow(\infty, \infty)} \frac{\left.a b s \operatorname{mean}_{G_{1}}(D(n))-\text { mean }_{G_{2}}(D(n))\right)}{\operatorname{mean}_{G_{1}}(D(n))+\text { mean }_{G_{2}}(D(n))} \\
& =\lim _{\left(\left|W_{1}\right|,\left|W_{2}\right|\right) \rightarrow(\infty, \infty)} \frac{a b s\left(\frac{\left|W_{1}\right|}{\left|V_{1}\right|}-\frac{\left|W_{2}\right|}{\left|V_{2}\right|}\right)}{\frac{\left|W_{1}\right|}{\left|V_{1}\right|}+\frac{\left|W_{2}\right|}{\left|V_{2}\right|}} .
\end{aligned}
$$

If we let $\left|W_{1}\right|=\left|W_{2}\right|$ and $\left|V_{1}\right|=\left|V_{2}\right|$, we obtain

$$
\lim _{\left(\left|W_{1}\right|,\left|W_{2}\right|\right) \rightarrow(\infty, \infty)} \frac{a b s\left(\frac{\left|W_{1}\right|}{\left|V_{1}\right|}-\frac{\left|W_{1}\right|}{\left|V_{1}\right|}\right)}{\frac{\left|W_{1}\right|}{\left|V_{1}\right|}+\frac{\left|W_{1}\right|}{\left|V_{1}\right|}}=0 .
$$

Giving us a limit of 0 . If we instead let $\left|W_{1}\right|=\left|W_{2}\right|^{2}$ and $\left|V_{1}\right|=\left|V_{2}\right|$, we obtain

$$
\lim _{\left(\left|W_{1}\right|,\left|W_{2}\right|\right) \rightarrow(\infty, \infty)} \frac{a b s\left(\frac{\left|W_{1}\right|}{\left|V_{1}\right|}-\frac{\left|W_{1}\right|^{2}}{\left|V_{1}\right|}\right)}{\frac{\left|W_{1}\right|}{\left|V_{1}\right|}+\frac{\left|W_{1}\right|^{2}}{\left|V_{1}\right|}}=1 .
$$

Giving us a limit of 1 . As more than one limit can be found the multivariate limit for $N S^{\prime}\left(G_{1}, G_{2}\right)$ is undefined. As $N S\left(G_{1}, G_{2}\right)$ is equal to $N S^{\prime}\left(G_{1}, G_{2}\right)$ plus the Canberra distances between other aggregated features, $N S\left(G_{1}, G_{2}\right)$ also has an undefined multivariate limit and is not Size Consistent.

\section{A.13 Proof of Claim 4.8}

Unweighted Netsimile is a Size Consistent statistic.

Proof. Netsimile is a Size Consistent statistic when applied to unweighted graphs with one caveat. We must assume that the Canberra distance between $s_{G_{1}}^{i}$ and $s_{G_{2}}^{i}$ is 0 if both $s_{G_{1}}^{i}$ and $s_{G_{2}}^{i}$ are equal to 0 , as this is the only point where the Canberra distance is not defined. W

As Unweighted Netsimile treats the graphs $G_{1}$ and $G_{2}$ as unweighted, we can replace the graphs with their unweighted adjacency matrices $\widehat{A}_{1}$ and $\widehat{A}_{2}$.

Because the standard moments used as aggregates and the Canberra distance can be expressed as algebraic functions (taking into account the caveat for Canberra distance), if the limits $\lim _{\left(\left|W_{1}\right|,\left|W_{2}\right|\right) \rightarrow(\infty, \infty)}$ and $\lim _{\left(\left|V_{1}\right|,\left|V_{2}\right| \rightarrow\left(\left|V^{*}\right|,\left|V^{*}\right|\right)\right.}$ of the inputs to these functions exist and are finite, then the limit of the Unweighted Netsimile exists and is finite as well by Lemma 3.1.

As the cells of $\widehat{A_{1}}$ and $\widehat{A}_{2}$ are the values of binomial samples, the $\operatorname{limits} \lim _{\left(\left|W_{1}\right|,\left|W_{2}\right|\right) \rightarrow(\infty, \infty)} \widehat{A_{1}}, \widehat{A_{2}}$ are $A_{1}, A_{2}$. Likewise, the limits $\lim _{\left(\left|V_{1}\right|,\left|V_{2}\right| \rightarrow\left(\left|V^{*}\right|,\left|V^{*}\right|\right)\right.} A_{1}, A_{2}$ are $A^{*}, A^{*}$. As all the unweighted statistics that make up the unweighted Netsimile are finite when calculated on a finite matrix $A^{*}$, the inputs to the Canberra distance and the graph aggregates are finite, and therefore the unweighted Netsimile is finite.

\section{A.14 Proof of Claim 4.10}

Mass Shift is a Size Consistent statistic.

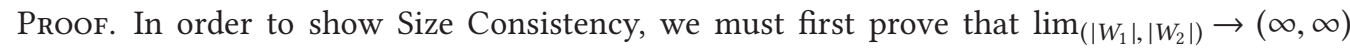
$\widehat{M S}\left(G_{1}, G_{2}\right)$ exists and is finite. The easiest way to prove this is to decompose the multivariate limit into single limits, which is made possible by utilizing Lemma 3.1.

Let $F\left(M_{1}, M_{2}\right)$ be a function for the squared difference of all cells in two matrices:

$$
F\left(M_{1}, M_{2}\right)=\frac{1}{Z} \sum_{i, j=1}^{m}\left(M_{1}[i, j]-M_{2}[i, j]\right)^{2},
$$


where $M_{1}, M_{2}$ are $m \times m$-sized matrices. We can then rewrite the empirical Mass Shift in terms of F:

$$
\widehat{M S}\left(G_{1}, G_{2}\right)=F\left(\frac{\widehat{P}_{1}}{\overline{\widehat{p}_{1}}}, \frac{\widehat{P}_{2}}{\overline{\widehat{p}_{2}}}\right) .
$$

Therefore, to prove that the empirical Mass Shift has a finite limit it is sufficient to show that $\lim _{\left|W_{1}\right| \rightarrow \infty} \frac{\widehat{p}_{1}}{\bar{p}_{1}}$ and $\lim _{\left|W_{2}\right| \rightarrow \infty} \frac{\widehat{P}_{2}}{{\overline{p_{2}}}_{2}}$ have finite limits. If we take the limit

$$
\operatorname{plim}_{\left(\left|W_{1}\right|,\left|W_{2}\right|\right) \rightarrow(\infty, \infty)} \frac{\widehat{\widehat{P}}_{1}}{\overline{\widehat{p_{1}}}},
$$

it can be simplified to

$$
\operatorname{plim}_{\left|W_{1}\right| \rightarrow \infty} \frac{\widehat{P}_{1}}{\overline{\widehat{p}_{1}}} .
$$

For any cell $p_{i j, 1} \in P_{1}$, we can write $\frac{\widehat{p}_{i j, 1}}{\widehat{p}_{1}}$ as

$$
\frac{\widehat{p}_{i j, 1}}{\widehat{\bar{p}}_{1}}=\left|\widehat{A_{1}}\right| \widehat{p}_{i j, 1}=\left|\widehat{A_{1}}\right| \frac{w_{i j, 1}}{\left|W_{1}\right|} .
$$

The term $\frac{w_{i j, 1}}{\left|W_{1}\right|}$ is the sample mean of a binomial distribution and will converge to $p_{i j, 1}$ as $\left|W_{1}\right| \rightarrow \infty$.

Likewise $\left|\widehat{A_{1}}\right| \rightarrow\left|A_{1}\right|$. If the value of any non-zero cell in $P_{1}$ is at least $\epsilon$, then the probability of not observing communication between a node pair in $P_{1}$ is $(1-\epsilon)^{\left|W_{1}\right|}$, which converges to 0 . Once every pair is observed $\left|\widehat{A_{1}}\right|=\left|A_{1}\right|$, so this term converges as well.

As both terms are converging, we can conclude that for any $i, j$ in $\widehat{P}_{1}, \frac{\widehat{p}_{i j, 1}}{\bar{p}_{1}} \rightarrow \frac{p_{i j, 1}}{\overline{p_{1}}}$, which means that

$$
\operatorname{plim}_{\left|W_{1}\right| \rightarrow \infty} \frac{\widehat{P_{1}}}{\overline{\widehat{p}_{1}}}=\frac{P_{1}}{\overline{\overline{p_{1}}}} .
$$

The argument for the other term is identical. As both terms have a finite limit, the limit of the empirical Mass Shift is also finite:

$$
\begin{aligned}
\lim _{\left(\left|W_{1}\right|,\left|W_{2}\right|\right) \rightarrow(\infty, \infty)} F\left(\frac{\widehat{P}_{1}}{\overline{\widehat{p}_{1}}}, \frac{\widehat{P}_{2}}{\widehat{\bar{p}_{2}}}\right) & =F\left(\frac{P_{1}}{\overline{p_{1}}}, \frac{P_{2}}{\overline{p_{2}}}\right) \\
& =M S\left(P_{1}, P_{2}\right) .
\end{aligned}
$$

Now, we need to show that the $\operatorname{limit}_{\left(\left|V_{1}\right|,\left|V_{2}\right|\right) \rightarrow\left(\left|V^{*}\right|,\left|V^{*}\right|\right)} M S\left(P_{1}, P_{2}\right)=M S\left(P_{1}^{*}, P_{2}^{*}\right)$. As before, we can transform the statistic using the squared matrix distance function, and all we need to show for Size Consistency is that

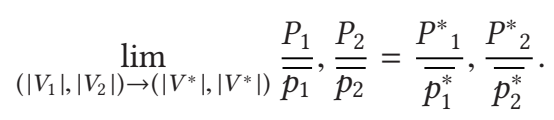

As the Mass Shift is a summation over only the nodes in the intersection of the two node sets: $V_{\cap}=V_{1} \cap V_{2}$ we can rewrite the multivariate limit as a single limit over $\left|V_{\cap}\right| \rightarrow\left|V^{*}\right|$. 
We will begin by showing the limit of the term $\frac{p_{1}}{\overline{p_{1}}}$. For any $i, j$, we can rewrite $\frac{p_{i j, 1}}{\overline{p_{1}}}$ as

$$
\begin{aligned}
\frac{p_{i j, 1}}{\overline{p_{1}}} & =p_{i j, 1} \sum_{i, j \in V_{\cap}} I\left[p_{i j, 1}>0\right] \\
& =\frac{p_{i j, 1}^{*} \sum_{i, j \in V_{\cap}} I\left[p_{i j, 1}^{*}>0\right]}{\sum_{i j \in V_{n}} p_{i j}^{*}} \\
& =\frac{p_{i j, 1}^{*}}{\overline{p_{1}^{*} \mid V_{\cap}}}
\end{aligned}
$$

where $\overline{p_{1}^{*}} \mid V_{\cap}$ is the sample mean of non-zero cells of $P^{*}$ that are members of $V_{\cap}$. As $\frac{1}{\overline{p_{1}^{*}} \mid V_{\cap}}$ is the inverse sample mean according to Slutsky's Theorem it converges to the true inverse mean $\frac{1}{\overline{p_{1}^{*}}}$, making the limit

$$
\lim _{\left|V_{n}\right| \rightarrow\left|V^{*}\right|} \frac{p_{i j, 1}}{\overline{p_{1}}}=\frac{p^{*}{ }_{i j, 1}}{\overline{p_{1}^{*}}}
$$

This means that

$$
\lim _{\left(\left|V_{1}\right|,\left|V_{2}\right|\right) \rightarrow\left(\left|V^{*}\right|,\left|V^{*}\right|\right)} F\left(\frac{P_{1}}{\overline{p_{1}}}, \frac{P_{2}}{\overline{p_{2}}}\right)=F\left(\frac{P^{*}}{\overline{p_{1}^{*}}}, \frac{P^{*}}{\overline{p_{2}^{*}}}\right)=M S\left(P_{1}^{*}, P_{2}^{*}\right),
$$

and the empirical Mass Shift is a Size Consistent Statistic with a limit of $M S\left(P_{1}^{*}, P_{2}^{*}\right)$.

Proof of Claim 4.11: The error of the empirical Mass Shift $\widehat{M S}\left(G_{1}, G_{2}\right)$ is less than or equal to the maximum error

$$
\epsilon_{\max }=\frac{1}{8\left|V^{*}\right|^{2}}\left(\frac{\left|V^{*}\right|\left(2 W-\left|V^{*}\right|+2\right)}{W}\right)^{2},
$$

and the error will be less than this bound with probability $4\left(\begin{array}{c}\left|V^{*}\right|-2 \\ \left|V^{*}\right|-V\end{array}\right) /\left(\begin{array}{c}\left|V^{*}\right| \\ \left|V^{*}\right|-V\end{array}\right)$.

Proof. The maximum error is attained when as much edge weight as possible lies on a node that does not follow the same edge distribution as the rest of the nodes in the graph. Consider a graph with a pair of "outlier" nodes and $\left|V^{*}\right|-2$ "normal" nodes, where normal nodes exist as disjoint pairs with an edge weight of 1 between pairs (the least amount of edge weight required to ensure that no node has a degree of 0 ) while the outlier nodes have all the remaining edge weight of the graph $\left(W-\frac{\left|V^{*}\right|+2}{2}\right)$ placed between them. Let $G_{1}$ and $G_{2}$ both be graphs of this type that are entirely identical except that the outlier nodes are a different pair of nodes. If the outlier nodes (at least one of each pair) are not part of the set $V$, then the empirical mass shift will be 0 rather than the correct answer, giving us a maximum error of

$$
\begin{aligned}
\epsilon_{\text {max }} & =\frac{1}{|V|^{2}} \sum_{i j \in V}\left(\frac{\widehat{p}_{i j, 1}}{\widehat{\bar{p}}_{1}}-\frac{\widehat{p}_{i j, 2}}{\widehat{\bar{p}}_{2}}\right)^{2} \\
& =\frac{2}{|V|^{2}}\left(\widehat{A} \frac{w_{i j}}{W}\right)^{2} \\
& =\frac{2}{\left|V^{*}\right|^{2}}\left(\frac{\left|V^{*}\right|}{2} \frac{\left(W-\frac{\left|V^{*}\right|+2}{2}\right)}{W}\right)^{2} \\
& =\frac{1}{8\left|V^{*}\right|^{2}}\left(\frac{\left|V^{*}\right|\left(2 W-\left|V^{*}\right|+2\right)}{W}\right)^{2} .
\end{aligned}
$$


In order to attain this error, at least one of each pair of outlier nodes must not be in the observed node set $V$. This gives us a probability $4\left(\begin{array}{c}\left|V^{*}\right|-2 \\ \left|V^{*}\right|-V\end{array}\right) /\left(\begin{array}{c}\left|V^{*}\right| \\ \left|V^{*}\right|-V\end{array}\right)$ that the error attains the maximum limit in the worst-case scenario.

\section{A.15 Derivation of Equation (10)}

As the expectation of $w_{i j, x}^{2}$ for any node pair $i, j$ given $\left|W_{x}\right|$ can be written as

$$
\begin{aligned}
E_{W_{x}}\left[w_{i j, x}^{2}\right] & =\operatorname{Var}\left(w_{i j, x}\right)+E_{W_{x}}\left[w_{i j, x}\right]^{2} \\
& =\operatorname{Var}\left(\operatorname{Bin}\left(\left|W_{x}\right|, p_{i j, x}\right)\right)+E_{W_{x}}\left[\operatorname{Bin}\left(\left|W_{x}\right|, p_{i j, x}\right)\right]^{2} \\
& =\left|W_{x}\right| p_{i j, x}\left(1-p_{i j, x}\right)+\left|W_{x}\right|^{2} p_{i j, x}^{2} .
\end{aligned}
$$

We can rewrite the expectation of the empirical mass shift:

$$
\begin{aligned}
& \frac{1}{Z_{P}} \sum_{i j \in V} \frac{\left(\left|A_{1}\right|\right)^{2}}{\left|W_{1}\right|^{2}}\left(\left|W_{1}\right| p_{i j, 1}\left(1-p_{i j, 1}\right)+\left|W_{1}\right|^{2} p_{i j, 1}^{2}\right)+\frac{\left(\left|A_{2}\right|\right)^{2}}{\left|W_{2}\right|^{2}}\left(\left|W_{2}\right| p_{i j, 2}\left(1-p_{i j, 2}\right)+\left|W_{2}\right|^{2} p_{i j, 2}^{2}\right) \\
& =\frac{1}{Z_{P}} \sum_{i j \in V}\left(\left|A_{1}\right| p_{i j, 1}-\left|A_{2}\right| p_{i j, 2}\right)^{2}+\left.\frac{1}{\left|W_{1}\right|}\left|\frac{\left|A_{2}\right|}{\left|W_{2}\right|}\right| W_{1}||_{1}\right|_{i j, 1} p_{i j, 1}\left|W_{2}\right| p_{i j, 2}\left(1-p_{i j, 1}\right)+\frac{1}{\left|W_{2}\right|}\left|A_{2}\right|^{2} p_{i j, 2}\left(1-p_{i j, 2}\right) \\
& =\operatorname{MS}\left(P_{1}, P_{2}\right)+\frac{1}{\left|W_{1}\right|}\left|A_{1}\right|^{2} p_{i j, 1}\left(1-p_{i j, 1}\right)+\frac{1}{\left|W_{2}\right|}\left|A_{2}\right|^{2} p_{i j, 2}\left(1-p_{i j, 2}\right)
\end{aligned}
$$

which is equal to $M S\left(P_{1}, P_{2}\right)$ plus a bias term.

\section{A.16 Proof of Claim 4.12}

Probability Degree Distance is a Size Consistent statistic.

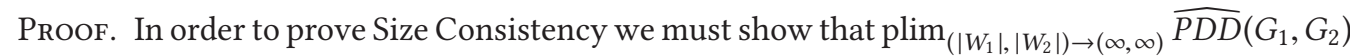
exists and is finite. Similar to Mass Shift, if we define a function $F$ that is the sum of the squared distances between bin counts we can transform the limit:

$$
\begin{aligned}
& \operatorname{plim}_{\left(\left|W_{1}\right|,\left|W_{2}\right|\right) \rightarrow(\infty, \infty)} \widehat{P D D}\left(G_{1}, G_{2}\right) \\
& =\operatorname{plim}_{\left(\left|W_{1}\right|,\left|W_{2}\right|\right) \rightarrow(\infty, \infty)} F\left(\frac{1}{\left|V_{1}\right|} \sum_{i \in V_{1}} I\left[\widehat{P D}_{i}\left(G_{1}\right) \in \operatorname{bin}_{k}\right], \frac{1}{\left|V_{2}\right|} \sum_{i \in V_{2}} I\left[\widehat{P D}_{i}\left(G_{2}\right) \in \operatorname{bin}_{k}\right]\right),
\end{aligned}
$$

and we need only show that the inputs to $F$ exist and are finite according to Lemma 3.1. We can rewrite the inputs:

$$
\begin{aligned}
& \lim _{\left|W_{x}\right| \rightarrow \infty} \frac{1}{\left|V_{x}\right|} \sum_{i \in V_{x}} I\left[\widehat{P D}_{i}\left(G_{x}\right) \in \operatorname{bin}_{k}\right] \\
= & \frac{1}{\left|V_{x}\right|} \sum_{i \in V_{x}} \lim _{\left|W_{x}\right| \rightarrow \infty} I\left[\widehat{P D}_{i}\left(G_{x}\right) \in \operatorname{bin}_{k}\right] \\
= & \frac{1}{\left|V_{x}\right|} \sum_{i \in V_{x}} \lim _{\left|W_{x}\right| \rightarrow \infty} I\left[\left|V_{x}\right| \sum_{j \in V_{x}} \frac{w_{i j, x}}{\left|W_{x}\right|} \in \text { bin }_{k}\right] .
\end{aligned}
$$


As the quantity $\frac{w_{i j, x}}{\left|W_{x}\right|}$ is the mean of a binomial variable, this converges to the underlying probability $p_{i j, x}$, making the limit

$$
\begin{aligned}
& \frac{1}{\left|V_{x}\right|} \sum_{i \in V_{x}} I\left[\left|V_{x}\right| \sum_{j \in V_{x}} p_{i j, x} \in \operatorname{bin}_{k}\right] \\
= & \frac{1}{\left|V_{x}\right|} \sum_{i \in V_{x}} I\left[P D\left(P_{x}\right) \in \operatorname{bin}_{k}\right] .
\end{aligned}
$$

This makes the limit of the empirical Probability Degree Distance:

$$
\begin{aligned}
\lim _{\left(\left|W_{1}\right|,\left|W_{2}\right|\right) \rightarrow(\infty, \infty)} \widehat{P D D}\left(G_{1}, G_{2}\right) & =F\left(\frac{1}{\left|V_{1}\right|} \sum_{i \in V_{1}} I\left[P D\left(P_{1}\right) \in \text { bin }_{k}\right], \frac{1}{\left|V_{2}\right|} \sum_{i \in V_{2}} I\left[P D\left(P_{2}\right) \in \text { bin }_{k}\right]\right) \\
& =P D D\left(P_{1}, P_{2}\right),
\end{aligned}
$$

which is finite. Now we must show that

$$
\lim _{\left(\left|V_{1}\right|,\left|V_{2}\right|\right) \rightarrow\left(\left|V^{*}\right|,\left|V^{*}\right|\right)} P D D\left(P_{1}, P_{2}\right)
$$

exists and is finite. We can rewrite this limit as

$$
\lim _{\left(\left|V_{1}\right|,\left|V_{2}\right|\right) \rightarrow\left(\left|V^{*}\right|,\left|V^{*}\right|\right)} F\left(\frac{1}{\left|V_{1}\right|} \sum_{i \in V_{1}} I\left[\left|V_{1}\right| \sum_{j \in V_{1}} p_{i j, 1} \in \operatorname{bin}_{k}\right], \frac{1}{\left|V_{2}\right|} \sum_{i \in V_{2}} I\left[\left|V_{2}\right| \sum_{j \in V_{2}} p_{i j, 2} \in \operatorname{bin}_{k}\right]\right) .
$$

As before, we need only show that the inputs to this function have a finite limit, which means we need to show that

$$
\lim _{V_{x} \rightarrow V^{*}} \frac{1}{\left|V_{x}\right|} \sum_{i \in V_{x}} I\left[\left|V_{x}\right| \sum_{j \in V_{x}} p_{i j, x} \in \operatorname{bin}_{k}\right]
$$

has finite limits. We can rewrite this as

$$
\lim _{\left|V_{x}\right| \rightarrow\left|V^{*}\right|} I\left[\frac{\left|V_{x}\right|}{\sum_{i j \in V_{x}} p_{i j, x}^{*}} \sum_{j \in V_{x}} p_{i j, x}^{*} \in b i n_{k}\right] .
$$

The ratio $\frac{\left|V_{x}\right|}{\sum_{i j \in V_{x}} p_{i j, x}^{*}}$ can be rewritten as $\frac{1}{\bar{p}^{*} \mid V_{x}}$, where $\bar{p}^{*} \mid V_{x}$ is the mean probability mass in $P^{*}$ of cells that belong to $V_{x}$. As this is an inverse mean, it will converge in probability to the true inverse mean $\frac{1}{\bar{p}^{*}}$, which is also equal to $\left|V^{*}\right|$. The summation $\sum_{j \in V_{x}} p_{i j, x}^{*}$ also converges to $\sum_{j \in V^{*}} p_{i j, x}^{*}$, leaving the limit as

$$
I\left[\left|V^{*}\right| \sum_{j \in V^{*}} p_{i j, x}^{*} \in \operatorname{bin}_{k}\right] .
$$

This means the limit on the function goes to

$$
\begin{aligned}
& \lim _{\left(\left|V_{1}\right|,\left|V_{2}\right|\right) \rightarrow\left(\left|V^{*}\right|,\left|V^{*}\right|\right)} F\left(\frac{1}{\left|V_{1}\right|} \sum_{i \in V_{1}} I\left[\left|V_{1}\right| \sum_{j \in V_{1}} p_{i j, 1} \in \operatorname{bin}_{k}\right], \frac{1}{\left|V_{2}\right|} \sum_{i \in V_{2}} I\left[\left|V_{2}\right| \sum_{j \in V_{2}} p_{i j, 2} \in \operatorname{bin}_{k}\right]\right) \\
& =F\left(I\left[\left|V^{*}\right| \sum_{j \in V^{*}} p_{i j, 1}^{*} \in \operatorname{bin}_{k}\right], I\left[\left|V^{*}\right| \sum_{j \in V^{*}} p_{i j, 2}^{*} \in \operatorname{bin}_{k}\right]\right)=\operatorname{PDD}\left(P_{1}^{*}, P_{2}^{*}\right),
\end{aligned}
$$

which is finite. 
Proof of Claim 4.13: : The error of the Probability Degree Distance is bounded by

$$
\frac{\left.2\left(\left|V^{*}\right|-1\right)^{2}\right)+2}{\left|V^{*}\right|^{2}}
$$

and the probability of attaining this error is at most $\left(\begin{array}{c}\left|V^{*}\right|-1 \\ \left|V^{*}\right|-|V|-1\end{array}\right) /\left(\begin{array}{c}\left|V^{*}\right| \\ \left|V^{*}\right|-|V|\end{array}\right)$.

Proof. The maximum error occurs when there is a "super-hub" node with a degree large enough to ensure all other nodes in the graph are in a different bin if it is part of the set $V$ than if it is not present. Consider the graph where all but one of the nodes are arranged as pairs with an edge weight of 1 , and with one hub node connected to all node of the graph with the remaining edge weight $W-\frac{\left|V^{*}\right|-1}{2}$ divided evenly among all of its edges. Let this graph be $G_{1}$, and let $G_{2}$ be a graph that is identical except that the hub node instead connects to one node that has all of the remaining edge weight. If the hub node is not observed the score produced is 0 , while the true value is

$$
\begin{aligned}
\widehat{P D} & =\sum_{b i n_{k} \in B i n s}\left(\frac{1}{\left|V^{*}\right|} \sum_{i \in V_{1}} I\left[\widehat{P D}_{i}\left(P_{1} \mid V^{*}\right) \in \operatorname{bin}_{k}\right]-\frac{1}{\left|V^{*}\right|} \sum_{i \in V^{*}} I\left[P D_{i}\left(P_{2} \mid V^{*}\right) \in \operatorname{bin}_{k}\right]\right)^{2} \\
& =\frac{1}{\left|V^{*}\right|^{2}}\left(\left(\left|V^{*}\right|-1\right)^{2}+1\right) .
\end{aligned}
$$

As all $\left|V^{*}\right|-1$ non-hub nodes will be in the same bin together, which is a different bin in $G_{1}$ and $G_{2}$, the hub node will be in its own bin, and the node that connects to the hub in $G_{2}$ will be in its own bin. This becomes the maximum error. The probability of this occurring if $V$ nodes are observed is the odds that the hub is not part of the set $V$, which is $\left(\begin{array}{c}\left|V^{*}\right|-1 \\ \left|V^{*}\right|-|V|-1\end{array}\right) /\left(\begin{array}{c}\left|V^{*}\right| \\ \left|V^{*}\right|-|V|\end{array}\right)$.

\section{A.17 Proof of Claim 4.14}

\section{TP is a Size Consistent statistic.}

As with Barrat Clustering, if we take the limit with increasing $|W|$ :

$$
\begin{aligned}
\lim _{|W| \rightarrow \infty} \frac{1}{Z} \sum_{i j k \in V} \widehat{|A|}^{3} \widehat{p}_{i j} \widehat{p}_{i k} \widehat{p}_{j k} & =\lim _{|W| \rightarrow \infty} \frac{1}{Z} \sum_{i j k \in V} \frac{\widehat{|A|}^{3}}{|W|^{3}} w_{i j} w_{i k} w_{j k} \\
= & \frac{1}{Z} \sum_{i j k \in V}|A|^{3} p_{i j} p_{i k} p_{j k}=T P(P) .
\end{aligned}
$$

Now if we take the limit as $|V| \rightarrow\left|V^{*}\right|$ :

$$
\begin{aligned}
& \lim _{|V| \rightarrow\left|V^{*}\right|} \frac{1}{Z} \sum_{i j k \in V}|A|^{3} p_{i j} p_{i k} p_{j k} \\
& =\lim _{|V| \rightarrow\left|V^{*}\right|} \frac{1}{Z} \sum_{i j k \in V} \frac{|A|^{3}}{\left(\sum_{i j \in V} p_{i j}^{*}\right)^{3}} p_{i j}^{*} p_{i k}^{*} p_{j k}^{*} \\
& =\lim _{|V| \rightarrow\left|V^{*}\right|} \frac{1}{Z} \sum_{i j k \in V} \frac{1}{\left(\overline{\left.p^{*} \mid V\right)^{3}}\right.} p_{i j}^{*} p_{i k}^{*} p_{j k}^{*} .
\end{aligned}
$$

Similar to the approach before, $\frac{1}{\left(\bar{p}^{*} \mid V\right)^{3}}$ converges to $\frac{1}{\left(\bar{p}^{*}\right)^{3}}$ by Slutsky's Theorem, so the final limit is

$$
=\frac{1}{Z^{*}} \sum_{i j k \in V^{*}} \frac{1}{\left(\bar{p}^{*}\right)^{3}} p_{i j}^{*} p_{i k}^{*} p_{j k}^{*}=T P\left(P^{*}\right) .
$$


As with the Mass Shift, let us take the expectation w.r.t. $|W|$ and see if we can improve the rate of convergence with a bias correction:

$$
\begin{aligned}
E[\widehat{T P}(G)] & =E\left[\frac{1}{Z} \sum_{i j k \in V}(\widehat{|A|})^{3} \widehat{p}_{i j} \widehat{p}_{i k} \widehat{p}_{j k}\right] \\
& =\frac{1}{Z} \sum_{i j k \in V} E\left[\left(\frac{\widehat{|A|}}{|W|}\right)^{3} w_{i j} w_{i k} w_{j k}\right] .
\end{aligned}
$$

As before, let us assume that we have enough edge samples so that $\widehat{|A|}=|A|$ :

$$
\frac{1}{Z} \sum_{i j k \in V} E\left[\left(\frac{|A|}{|W|}\right)^{3} w_{i j} w_{i k} w_{j k}\right] \text {. }
$$

The quantity $E\left[\frac{1}{|W|^{3}} w_{i j} w_{i k} w_{j k}\right]$ can be calculated with

$$
\begin{aligned}
& E\left[\frac{1}{|W|^{3}} w_{i j} w_{i k} w_{j k}\right]=\frac{1}{|W|^{3}} E\left[w_{i j} w_{i k} w_{j k}\right] \\
& \quad=\frac{1}{|W|^{3}} E\left[w_{i j} w_{i k}\right] E\left[w_{j k}\right]-\operatorname{cov}\left(w_{i j} w_{i k}, w_{j k}\right) \\
& \quad=\frac{1}{|W|^{3}} E\left[w_{i j}\right] E\left[w_{i k}\right] E\left[w_{j k}\right]-E\left[w_{j k}\right] \operatorname{cov}\left(w_{i j}, w_{i k}\right)-\operatorname{cov}\left(w_{i j} w_{i k}, w_{j k}\right) \\
& =\frac{1}{|W|^{3}}|W|^{3} p_{i j} p_{i k} p_{j k}+|W|^{2} p_{i j} p_{i k} p_{j k}-\operatorname{cov}\left(w_{i j} w_{i k}, w_{j k}\right) .
\end{aligned}
$$

The covariance term can be expanded with the formula for products of random variables [8]:

$$
\begin{aligned}
\operatorname{cov}\left(w_{i j} \cdot w_{i k}, w_{j k}\right)= & E\left[w_{i j}\right] \operatorname{cov}\left(w_{i k}, w_{j k}\right)+E\left[w_{i k}\right] \operatorname{cov}\left(w_{i j} w_{j k}\right)+E\left[\left(w_{i j}-E\left[w_{i j}\right]\right)\left(w_{i k}-E\left[w_{i k}\right]\right)\left(w_{j k}-E\left[w_{j k}\right]\right)\right] \\
= & -2|W|^{2} p_{i j} p_{i k} p_{j k}+E\left[w_{i j} w_{i k} w_{j k}-E\left[w_{i j}\right] w_{i k} w_{j k}-E\left[w_{i k}\right] w_{i j} w_{j k}-E\left[w_{j k}\right] w_{i k} w_{i j}\right. \\
& \left.+E\left[w_{i j}\right] E\left[w_{i k}\right] w_{j k}+E\left[w_{j k}\right] E\left[w_{i k}\right] w_{i j}+E\left[w_{i j}\right] E\left[w_{j k}\right] w_{i k}-E\left[w_{i j}\right] E\left[w_{j k}\right] E\left[w_{i k}\right]\right] \\
= & -2|W|^{2} p_{i j} p_{i k} p_{j k}+E\left[w_{i j} w_{i k} w_{j k}\right]-|W| p_{i j} E\left[w_{i k} w_{j k}\right]-|W| p_{i k} E\left[w_{i j} w_{j k}\right]-|W| p_{j k} E\left[w_{i k} w_{i j}\right] \\
& +3|W|^{3} p_{i j} p_{i k} p_{j k}-|W|^{3} p_{i j} p_{i k} p_{j k} \\
= & -2|W|^{2} p_{i j} p_{i k} p_{j k}+E\left[w_{i j} w_{i k} w_{j k}\right]-3|W|^{3} p_{i j} p_{i k} p_{j k}+|W| p_{i j} \operatorname{cov}\left(w_{i k}, w_{j k}\right) \\
& +|W| p_{i k} \operatorname{cov}\left(w_{i j}, w_{j k}\right)+|W| p_{j k} \operatorname{cov}\left(w_{i k}, w_{i j}\right)+3|W|^{3} p_{i j} p_{i k} p_{j k}-|W|^{3} p_{i j} p_{i k} p_{j k} \\
= & -2|W|^{2} p_{i j} p_{i k} p_{j k}+E\left[w_{i j} w_{i k} w_{j k}\right]-3|W|^{2} p_{i j} p_{i k} p_{j k}-|W|^{3} p_{i j} p_{i k} p_{j k} \\
= & -5|W|^{2} p_{i j} p_{i k} p_{j k}+E\left[w_{i j} w_{i k} w_{j k}\right]-|W|^{3} p_{i j} p_{i k} p_{j k} .
\end{aligned}
$$

By plugging the covariance into the original equation, we obtain:

$$
\begin{aligned}
E\left[\frac{1}{|W|^{3}} w_{i j} w_{i k} w_{j k}\right] & =\frac{1}{|W|^{3}}\left(|W|^{3} p_{i j} p_{i k} p_{j k}+|W|^{2} p_{i j} p_{i k} p_{j k}+5|W|^{2} p_{i j} p_{i k} p_{j k}-E\left[w_{i j} w_{i k} w_{j k}\right]+|W|^{3} p_{i j} p_{i k} p_{j k}\right) \\
2 E\left[\frac{1}{|W|^{3}} w_{i j} w_{i k} w_{j k}\right] & =\frac{1}{|W|^{3}}\left(2|W|^{3} p_{i j} p_{i k} p_{j k}+6|W|^{2} p_{i j} p_{i k} p_{j k}\right) \\
E\left[\frac{1}{|W|^{3}} w_{i j} w_{i k} w_{j k}\right] & =\frac{1}{|W|^{3}}\left(|W|^{3} p_{i j} p_{i k} p_{j k}+3|W|^{2} p_{i j} p_{i k} p_{j k}\right) \\
& =p_{i j} p_{i k} p_{j k}+\frac{3}{|W|} p_{i j} p_{i k} p_{j k} .
\end{aligned}
$$

So the bias term is $\frac{3}{|W|} p_{i j} p_{i k} p_{j k}$. If we subtract the empirical version of this term to compensate, we obtain the corrected empirical TP:

$$
\widehat{T P}(G)=\frac{1}{Z} \sum_{i j k \in V} \widehat{|A|}^{3}\left(\widehat{p}_{i j} \widehat{p}_{i k} \widehat{p}_{j k}-\frac{3}{|W|} \widehat{p}_{i j} \widehat{p}_{i k} \widehat{p}_{j k}\right) .
$$


Proof of Claim 4.15. The error of the empirical TP is bounded by

$$
\left(\frac{\left(2\left|V^{*}\right|+3\right)\left(2 W-\left|V^{*}\right|+3\right)}{12 W\left|V^{*}\right|}\right)^{3}
$$

and the probability that it achieves this bound is less than or equal to $1-\left(\begin{array}{c}\left|V^{*}\right|-3 \\ |V|-3\end{array}\right) /\left(\begin{array}{c}\left|V^{*}\right| \\ |V|\end{array}\right)$.

Proof. The worst-case scenario in terms of maximum error and probability of attaining that maximum error occurs when most of the edge weight lies on a single triangle. Consider a graph with $\left|V^{*}\right|$ nodes consisting of three "outlier" nodes with the rest of the nodes being "normal." Normal nodes are arranged in pairs with an edge weight of 1 between them (as nodes without any edges are not considered part of the graph), while all remaining edge weight $\left(W-\frac{\left|V^{*}\right|-3}{2}\right)$ is evenly distributed between the three outlier nodes. If the TP is calculated on a subset $V$ of the nodes, which does not contain all three of the outliers, the score will be 0 , while the true score is

$$
\begin{aligned}
T P & =\frac{1}{\left|V^{*}\right|^{3}}\left(\frac{\left|V^{*}\right|-3}{2}+3\right)^{3}\left(\frac{2 W-\left|V^{*}\right|+3}{6 W}\right)^{3} \\
& =\left(\frac{\left(2\left|V^{*}\right|+3\right)\left(2 W-\left|V^{*}\right|+3\right)}{12 W\left|V^{*}\right|}\right)^{3},
\end{aligned}
$$

which is also the maximum error. As the maximum error is only achieved when all three outlier nodes are part of the set $V$, the probability of attaining the maximum error is less than or equal to $1-\left(\begin{array}{c}\left|V^{*}\right|-3 \\ |V|-3\end{array}\right) /\left(\begin{array}{c}\left|V^{*}\right| \\ |V|\end{array}\right)$.

\section{ACKNOWLEDGMENTS}

The US Government is authorized to reproduce and distribute reprints for governmental purposes notwithstanding any copyright notation hereon. The views and conclusions contained herein are those of the authors and should not be interpreted as necessarily representing the official policies or endorsements either expressed or implied, of NSF or the US Government.

\section{REFERENCES}

[1] W. Aiello, F. Chung, and L. Lu. 2000. A random graph model for massive graphs. In Symposium on Theory of Computing.

[2] L. Akoglu, H. Tong, and D. Koutra. 2015. Graph based anomaly detection and description: A survey. Data Mining and Knowledge Discovery 29, 3 (2015), 626-688.

[3] A. Anagnostopoulos, R. Kumar, and M. Mahdian. 2008. Influence and correlation in social networks. In SIGKDD International Conference on Knowledge Discovery and Data Mining. 7-15.

[4] S. Aral, L. Muchnika, and A. Sundararajan. 2009. Distinguishing influence-based contagion from homophily-driven diffusion in dynamic networks. Proceedings of the National Academy of Sciences 106, 51 (2009), 21544-21549.

[5] L. Backstrom and others. 2006. Group formation in large social networks: Membership, growth, and evolution. In SIGKDD International Conference on Knowledge Discovery and Data Mining. 44-54.

[6] D. Banks and K. Carley. 1996. Models for network evolution. The fournal of Mathematical Sociology 21, 1-2 (1996), 173-196.

[7] M. Berlingerio, D. Koutra, and C. Faloutsos. 2012. Network similarity via multiple social theories. In International Conference on Advances in Social Networks Analysis and Mining.

[8] G. Bohrnstedt and A. Goldberger. 1969. On the exact covariance of products of random variables. Fournal of the American Statistical Association 64, 328 (1969), 1430-1442.

[9] C. Borgs, J. Chayes, L. Lovász, V. Sós, B. Szegedy, and K. Vesztergombi. 2006. Graph limits and parameter testing. In ACM Symposium on Theory of Computing.

[10] K. Borner, J. Maru, and R. Goldstone. 2004. The simultaneous evolution of author and paper networks. Proceedings on the National Academy of Sciences 101 (2004), 5266-5273.

[11] K. Borner, S. Sanyal, and A. Vespignani. 2007. Network science. Annual Review of Information Science and Technology 41 (2007), 537-607. 
[12] J. Boyd, W. Fitzgerald, and R. Beck. 2006. Computing core/periphery structures and permutation tests for social relations data. Social Networks 28 (2006), 165-178.

[13] H. Bunke, P. Dickinson, A. Humm, C. Irniger, and M. Kraetzl. 2006. Computer network monitoring and abnormal event detection using graph matching and multidimensional scaling. In Industrial Conference on Data Mining. Springer, Berlin, Heidelberg, 576-590.

[14] R. S. Burt. 2000. Decay functions. Social Networks 22 (2000), 1-28.

[15] N. Contractor, S. Wasserman, and K. Faust. 2006. Testing multitheoretical, multilevel hypotheses about organizational networks: An analytic framework and empirical example. Academy of Management Review 31, 3 (2006), 681-703.

[16] B. Desmarais and S. Cranmer. 2012. Micro-level interpretation of exponential random graph models with application to estuary networks. Policy Studies fournal 40, 3 (2012), 402-434.

[17] M. Dow and J. Cheverud. 1985. Comparison of distance matrices in studies of population structure and genetic microdifferentiation: Quadratic assignment. American fournal of Physical Anthropology 68, 3 (1985), 367-373.

[18] D. Lazer et al. 2010. The coevolution of networks and political attitudes. Political Communications 27, 3 (2010), 248-274.

[19] K. Faust and J. Skvoretz. 2002. Comparing networks across space and time, size and species. Sociological Methodology 32, 1 (2002), 267-299.

[20] T. La Fond and J. Neville. 2010. Randomization tests for distinguishing social influence and homophily effects. In World Wide Web Conference.

[21] B. Gallagher. 2006. Matching structure and semantics: A survey on graph-based pattern matching. Association for the Advancement of Artificial Intelligence AAAI FS 6 (2006), 45-53.

[22] B. Gao, D. Xiao, and X. Li. 2010. A survey of graph edit distance. Fournal of Pattern Analysis and Applications 13, 1 (2010), 113-129.

[23] C. Gao and J. Lafferty. 2017. Testing for global network structure using small subgraph statistics. arXiv Preprint arXiv:1710.00862 (2017).

[24] M. Gaston, M. Kraetzl, and W. Wallis. 2006. Using graph diameter for change detection in dynamic networks. Australasian fournal of Combinatorics 35 (2006), 299.

[25] S. Hanneke, W. Fu, and E. Xing. 2010. Discrete temporal models of social networks. Electronic fournal of Statistics 4 (2010), 585-605.

[26] R. Hanneman and M. Riddle. 2005. Introduction to Social Network Methods. University of California Riverside.

[27] K. Henderson and others. 2012. RolX: Structural role extraction \& mining in large graphs. In SIGKDD International Conference on Knowledge Discovery and Data Mining.

[28] P. Holme, S. Park, B. Kim, and C. Edling. 2007. Korean university life in a network perspective: Dynamics of a large affiliation network. Physica A: Statistical Mechanics and its Applications 373 (2007), 821-830.

[29] D. Koutra, J. Vogelstein, and C. Faloutsos. 2013. DELTACON: A principled massive-graph similarity function. In Proceedings of the SIAM International Conference in Data Mining.

[30] D. Krackhardt. 1988. Predicting with networks: Nonparametric multiple regression analysis of dyadic data. Social Networks 10, 4 (1988), 359-381.

[31] T. LaFond, J. Neville, and B. Gallagher. 2014. Anomaly detection in networks with changing trends. In Outlier Detection and Description under Data Diversity at the International Conference on Knowledge Discovery and Data Mining.

[32] J. Leskovec. 2005. Realistic, mathematically tractable graph generation and evolution, using Kronecker multiplication. In European Conference on Machine Learning and Principles and Practice of Knowledge Discovery in Databases. 133-145.

[33] R. Madhavan, B. R. Koka, and J. E. Prescott. 1998. Networks in transition: How industry events (re)shape interfirm relationships. Strategic Management fournal 19 (1998), 439-459.

[34] I. McCulloh. 2009. Detecting Changes in a Dynamic Social Network. Technical Report CMU-ISR-09-104.

[35] M. Mongiovı, P. Bogdanov, R. Ranca, E. Papalexakis, C. Faloutsos, and A. Singh. 2013. Netspot: Spotting significant anomalous regions on dynamic networks. In SIAM International Conference on Data Mining.

[36] S. Moreno and J. Neville. 2013. Network hypothesis testing using mixed kronecker product graph models. International Conference on Data Mining (2013).

[37] S. Moreno, J. Neville, and S. Kirshner. 2013. Learning mixed kronecker product graph models with simulated method of moments. In SIGKDD International Conference on Knowledge Discovery and Data Mining.

[38] J. Onnela, J. Saramäki, J. Kertész, and K. Kaski. 2005. Intensity and coherence of motifs in weighted complex networks. Physical Review 71, 6 (2005), 065103

[39] P. Papadimitriou, A. Dasdan, and H. Garcia-Molina. 2010. Web graph similarity for anomaly detection. Journal of Internet Services and Applications 1, 1 (2010), 19-30.

[40] J. Pfeiffer, T. LaFond, S. Moreno, and J. Neville. 2012. Fast generation of large scale social networks while incorporating transitive closures. In IEEE SocialCom. 
[41] C. Priebe, J. Conroy, D. Marchette, and Y. Park. 2005. Scan statistics on enron graphs. Computational and Mathematical Organization Theory 11, 3 (2005), 229-247.

[42] A. Sanil, D. Banks, and K. Carley. 1995. Models for evolving fixed node networks: Model fitting and model testing. Social Networks 17 (1995), 65-81.

[43] J. Saramäki, M. Kivelä, J.-P. Onnela, K. Kaski, and J. Kertesz. 2007. Generalizations of the clustering coefficient to weighted complex networks. Physical Review (2007).

[44] P. Shoubridge, M. Kraetzl, and D. Ray. 1999. Detection of abnormal change in dynamic networks. In Information, Decision and Control. 22, 2 (1999), 161-170.

[45] S. Snijders. 1999. Non-parametric standard errors and tests for network statistics. Connections (1999), 215-247.

[46] T. Snijders. 2005. Models for longitudinal network data. Models and Methods in Social Network Analysis 1 (2005).

[47] T. Snijders and others. 2006. New specifications for exponential random graph models. Sociological Methodology 36, 1 (2006), 99-153.

[48] T. Snijders, C. Steglich, and M. Schweinberger. 2007. Modeling the co-evolution of networks and behavior. Longitudinal Models in the Behavioral and Related Sciences 31, 4 (2007), 397-404.

[49] T. Snijders, G. Van de Bunt, and C. Steglich. 2010. Introduction to stochastic actor-based models for network dynamics. Social Networks 32, 1 (2010), 44-60.

[50] J. Wang and C. Q. Chiu. 2005. Detecting online auction inflated-reputation behaviors using social network analysis. North American Association for Computational Social and Organizational Sciences (2005).

[51] Yasser Yasami and Farshad Safaei. 2017. A statistical infinite feature cascade-based approach to anomaly detection for dynamic social networks. Computer Communications 100 (2017), 52-64.

[52] P. Yates and N. Mukhopadhyay. 2013. An inferential framework for biological network hypothesis tests. BMC Bioinformatics 14, 1 (2013), 94.

[53] J. Young. 2011. How do they 'end up together'? A social network analysis of self-control, homophily, and adolescent relationships. Journal of Quantitative Criminology 27 (2011), 251.

[54] T. Zimmerman and N. Nagappan. 2008. Predicting defects using network analysis on dependency graphs. In International Conference on Software Engineering.

Received July 2017; revised January 2018; accepted February 2018 